\title{
Dark-matter bound states from Feynman diagrams
}

\author{
Kalliopi Petraki, ${ }^{a}$ Marieke Postma ${ }^{a}$ and Michael Wiechers ${ }^{a, b}$ \\ ${ }^{a}$ Nikhef, Science Park 105, \\ 1098 XG Amsterdam, The Netherlands \\ ${ }^{b}$ GRAPPA Institute, University of Amsterdam, \\ Science Park 904, 1090 GL Amsterdam, The Netherlands \\ E-mail: kpetraki@nikhef.nl, mpostma@nikhef.nl, mwiecher@nikhef.nl
}

ABSTRACT: If dark matter couples directly to a light force mediator, then it may form bound states in the early universe and in the non-relativistic environment of haloes today. In this work, we establish a field-theoretic framework for the computation of bound-state formation cross-sections, de-excitation and decay rates, in theories with long-range interactions. Using this formalism, we carry out specific computations for scalar particles interacting either via a light scalar or vector mediator. At low relative velocities of the interacting particles, the formation of bound states is enhanced by the Sommerfeld effect. For particle-antiparticle pairs, we show that bound-state formation can be faster than annihilation into radiation in the regime where the Sommerfeld effect is important. The field-theoretic formalism outlined here can be generalised to compute bound-state formation cross-sections in a variety of theories, including theories featuring non-Abelian (albeit non-confining) interactions, such as the electroweak interactions.

Keywords: Beyond Standard Model, Cosmology of Theories beyond the SM, Nonperturbative Effects

ArXiv EPrint: 1505.00109 


\section{Contents}

1 Introduction 1

2 Bound-state and two-particle state wavefunctions 5

2.1 The Bethe-Salpeter wavefunctions 5

2.2 The 4-point Green's function and Dyson-Schwinger equation $\quad 7$

2.3 Completeness relation and decomposition of the 4-point function 8

2.4 The Bethe-Salpeter equation for bound and scattering states 10

$\begin{array}{ll}2.5 & \text { Normalization of the Bethe-Salpeter wavefunctions } \\ \end{array}$

2.6 The instantaneous approximation and the Schrödinger equation 13

$\begin{array}{ll}2.7 & \text { Normalization of the Schrödinger wavefunctions } \\ \end{array}$

3 Radiative level transitions $\quad 16$

$\begin{array}{ll}3.1 \text { The 5-point Green's function } & 17\end{array}$

$\begin{array}{lll}3.2 & \text { Transition amplitudes } & 19\end{array}$

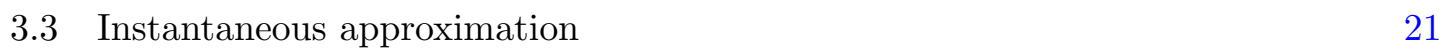

$\begin{array}{lll}3.4 & \text { On-shell approximation } & 22\end{array}$

$\begin{array}{lll}3.5 & \text { Bound-state formation cross-sections } & 23\end{array}$

3.6 Bound-state de-excitation rates 24

4 Decay of unstable bound states and (co-)annihilation of unbound pairs 24

4.1 Non-perturbative amplitude 24

$\begin{array}{lll}4.2 & \text { On-shell approximation } & 26\end{array}$

4.3 Two-body (co-)annihilation cross-sections and bound-state decay rates 26

5 Bound-state formation, de-excitation and decay rates for specific inter$\begin{array}{ll}\text { actions } & \mathbf{2 8}\end{array}$

$\begin{array}{lll}5.1 & \text { Scalar mediator } & 30\end{array}$

$\begin{array}{lll}\text { 5.1.1 Bound-state formation amplitudes } & 31\end{array}$

5.1.2 Bound-state formation cross-sections and partial-wave unitarity 34

$\begin{array}{lll}5.1 .3 & \text { De-excitation rate } & 35\end{array}$

5.1.4 Annihilation vs bound-state formation for particle-antiparticle pairs 36

5.1.5 Particle-antiparticle bound-state decay rates 36

5.2 Vector mediator 37

5.2.1 Bound-state formation amplitude, cross-section and partial-wave unitarity 38

5.2.2 Annihilation vs bound-state formation for particle-antiparticle pairs $\quad 39$

5.2.3 Particle-antiparticle bound-state decay rate $\quad 40$

$\begin{array}{lll}6 & \text { Discussion } & 40\end{array}$

A Bethe-Salpeter wavefunctions: summary of definitions 42 
C Calculation of $\mathcal{S}_{0}(\mathrm{p} ; P), \mathcal{S}(t ; \mathrm{p} ; P)$ and $\mathcal{S}_{1,2}(t ; \mathrm{p} ; P)$

D Partial-wave analysis for (co-)annihilation and decay processes

E Integrals for the non-relativistic reduction of transition amplitudes

F Schrödinger wavefunctions and convolution integrals

$\begin{array}{lll}\text { F.1 Solutions of the Schrödinger equation } & 50\end{array}$

$\begin{array}{lll}\text { F.2 Convolution of the wavefunctions } & 51\end{array}$

\section{Introduction}

Dark matter (DM) with long-range interactions, mediated by a light or massless force carrier, appears in diverse theories motivated on various grounds. Let us mention a few important examples. Dark matter with sizable self-interactions, mediated by a light particle, can explain the observed galactic structure better than collisionless DM [1-10]. Asymmetric DM [11-14] - motivated in part by the similarity of the dark and the ordinary matter abundances - resides, in most implementations, in a hidden sector that often includes long-range interactions [15-20]. Dark matter which dissipates energy via its coupling to a light force mediator may provide a dynamical explanation for some of the observed scaling relations governing haloes [21-24], and other features [25, 26]. Inside haloes, the inelastic scatterings of either symmetric or asymmetric DM with long-range interactions can produce radiative signals [27-35], which can potentially account for anomalous excesses observed in the radiation backgrounds [27-32]. Moreover, long-range DM-nucleon scattering implies a different interpretation of the direct-detection data than the commonly assumed shortrange scattering [36-39]. Notably, the long-range character of DM interactions is relevant not only for theories involving hidden sectors; even the electroweak interactions of the Standard Model manifest as long-range if DM is heavier than a few TeV [40-42]. Clearly, long-range interactions play a central role in the venture to identify DM. In order to extract accurate predictions for the DM phenomenology, it is then essential to fully understand their implications.

Long-range interactions typically imply the existence of bound states. The formation of DM bound states in the early universe and/or in the dense environment of haloes today affects the phenomenology of DM in many important ways. In the early universe, symmetric DM may form unstable bound states, whose decay contributes to the DM annihilation rate and affects the DM relic abundance [43]. Asymmetric DM may form stable bound states [15-20, 34, 44-52], which affect all manifestations of DM today, including the DM self-scattering in haloes [19, 46-48], the expected indirect-detection [29-34] and directdetection [53] signals, as well as the kinetic decoupling of DM from dark radiation [54]. Inside haloes, DM bound states — whether they are stable or unstable - may form 
radiatively and yield detectable signatures [28-30, 35]. Radiative signals may also be produced in transitions between the bound-state energy levels [31-33], or other related inelastic processes [34]. Moreover, the formation of DM bound states may be detectable at the LHC [55]. These rich phenomenological implications strongly suggest that it is critical to accurately account for the formation of DM bound states, in order to constrain the DM properties and eventually detect DM. This work is a step toward this goal.

There are, of course, two different classes of bound states: those arising due to nonconfining interactions, such as the atomic bound states in QED, and those arising due to confining interactions, such as the hadrons in QCD. Particles charged under a confining force always combine into hadronic states, roughly once the kinetic energy in their centerof-mass frame (or the temperature of their plasma) drops below the confinement scale. On the other hand, in the case of non-confining interactions, the efficiency of boundstate formation (BSF) depends on the corresponding cross-sections and the details of the thermodynamic environment. Here we shall consider bound states due to non-confining interactions, and calculate the cross-sections for their formation in the non-relativistic regime, which is relevant for cosmology and DM indirect detection signals. ${ }^{1}$

The formation of DM bound states in the early universe and inside the non-relativistic environment of haloes differs from BSF in colliders in some important ways. In high-energy colliders, the initial-state particles are highly relativistic. ${ }^{2}$ However, BSF is more efficient when the relative velocity of the interacting particles is lower than the expectation value of the relative velocity of the particles inside the bound state. Equivalently, this is when the kinetic energy in the center-of-mass $(\mathrm{CM})$ frame is lower than the binding energy; clearly, this lies within the non-relativistic regime. In this regime, the long-range interaction distorts the wavefunctions of the incoming particles, which cannot be approximated by momentum eigenstates (plane waves). This is the well-known Sommerfeld effect [62]. If the interaction is attractive, the Sommerfeld effect enhances the cross-section for any process the two particles may participate in, including the formation of bound states. It follows that, cosmologically, DM bound states form most efficiently after the temperature of the dark plasma drops below the binding energy. While the formation of bound states in the early universe eventually freezes out due to the cosmological expansion, bound states may again form efficiently in today's dense and non-relativistic haloes. In either case, to accurately estimate the formation of DM bound states, we must account for the Sommerfeld effect, which is a non-perturbative phenomenon, as is of course the very existence of bound states.

In this paper, we establish a field-theoretic framework for the calculation of BSF crosssections and other level-transition rates, as well as the decay rates of unstable bound states. Then, we carry out computations for specific interactions. We organise our work as follows.

We begin, in section 2, with reviewing how to determine the wavefunctions of the two-particle states and the bound states in the presence of a long-range interaction. We derive the Bethe-Salpeter equation for the wavefunctions, and reduce it to the Schrödinger

\footnotetext{
${ }^{1}$ Another class of bound states - non-topological solitons - has also been considered in the context of DM $[2,56-60]$.

${ }^{2}$ See, however, ref. [61].
} 


\begin{tabular}{|c|c|c|}
\hline & $\begin{array}{c}\text { Scalar mediator } \\
\alpha=g_{1} g_{2} /(16 \pi), \quad g_{1} g_{2}>0\end{array}$ & $\begin{array}{c}\text { Vector mediator } \\
\alpha=-c_{1} c_{2} g^{2} /(4 \pi), \quad c_{1} c_{2}<0\end{array}$ \\
\hline \multirow{2}{*}{$\sigma_{\mathrm{BSF}}^{\{100\}} v_{\mathrm{rel}} / \sigma_{c}$} & $\begin{array}{l}\text { degenerate species: } g_{1}=g_{2}, \eta_{1}=\eta_{2}=1 / 2 \\
\qquad \frac{2^{6} \alpha^{2}}{15} \frac{\zeta^{2}\left(3+2 \zeta^{2}\right)}{\left(1+\zeta^{2}\right)^{2}} e^{-4 \zeta \operatorname{arccot} \zeta}\end{array}$ & {$\left[\left(\eta_{2} c_{1}-\eta_{1} c_{2}\right)^{2}\right] 2^{7} \zeta^{4} e^{-4 \zeta \operatorname{arccot} \zeta}$} \\
\hline & $\begin{array}{c}\text { non-degenerate species } \\
{\left[\frac{\left(g_{1} \eta_{2}-g_{2} \eta_{1}\right)^{2}}{16 \pi \alpha}\right] \frac{2^{6} \zeta^{4} e^{-4 \zeta \operatorname{arccot} \zeta}}{3\left(1+\zeta^{2}\right)^{2}}}\end{array}$ & $3\left(1+\zeta^{2}\right)^{2}$ \\
\hline$\sigma_{\mathrm{BSF}}^{\{210\}} v_{\mathrm{rel}} / \sigma_{c}$ & {$\left[\frac{\left(g_{1} \eta_{2}+g_{2} \eta_{1}\right)^{2}}{16 \pi \alpha}\right] \frac{2^{7} \zeta^{6}\left(28+23 \zeta^{2}\right)}{15\left(4+\zeta^{2}\right)^{4}} e^{-4 \zeta \operatorname{arccot}(\zeta / 2)}$} & \\
\hline$\sigma_{\text {ann }} v_{\text {rel }} / \sigma_{c}$ & 1 & $\frac{1}{2}$ \\
\hline$\Gamma_{\{100\} \rightarrow \varphi \varphi}$ & $\mu \alpha^{5}$ & $\frac{\mu \alpha^{5}}{2}$ \\
\hline$\Gamma_{\{210\} \rightarrow \varphi \varphi}$ & $\frac{\mu \alpha^{7}}{2^{7} 3}$ & \\
\hline$\Gamma_{\{210\} \rightarrow\{100\}+\varphi}$ & $\frac{2^{7} \mu \alpha^{5}}{3^{7}}\left[\frac{\left(g_{1} \eta_{2}+g_{2} \eta_{1}\right)^{2}}{16 \pi \alpha}\right]$ & \\
\hline
\end{tabular}

Table 1. Summary of the bound-state-formation and annihilation cross-sections, the decay and de-excitation rates, computed in section 5 , for scalar particles interacting via a light scalar or vector mediator. The annihilation cross-sections and the rates of decay into $\varphi$-mediators refer to unbound and bound particle-antiparticle pairs respectively; all other formulae apply to any pair of particles. The cross-sections are normalised to $\sigma_{c} \equiv\left(\pi \alpha^{2} / \mu^{2}\right) \times 2 \pi \zeta /\left(1-e^{-2 \pi \zeta}\right)$, where $\alpha$ is the fine-structure constant entering the Coulomb potential, $\mu=m_{1} m_{2} /\left(m_{1}+m_{2}\right)$ is the reduced mass of the interacting species, and $\zeta=\alpha / v_{\text {rel }}$, with $v_{\text {rel }}$ being the relative velocity of the incoming particles. Also, $\eta_{1,2}=m_{1,2} /\left(m_{1}+m_{2}\right)$, and $g_{1,2}, c_{1,2}$ are the couplings of the interacting particles to the force mediators, as described by the Lagrangian densities of section 5 . In our computations, we have neglected the mediator mass. Unitarity suggests that the range of validity of the above computations is $\alpha \lesssim 0.5$.

equation using the instantaneous approximation in the non-relativistic regime. In section 3 , we determine the amplitudes for transitions between energy levels with the emission of a force mediator; this includes the radiative capture to a bound state. In the fully relativistic regime, we express the amplitudes for such processes in terms of the Bethe-Salpeter wavefunctions describing the initial and final states, and a perturbative interaction. We then employ the instantaneous and the non-relativistic approximations, to express the transition amplitudes in terms of the Schrödinger wavefunctions. In section 4, we repeat the analysis 

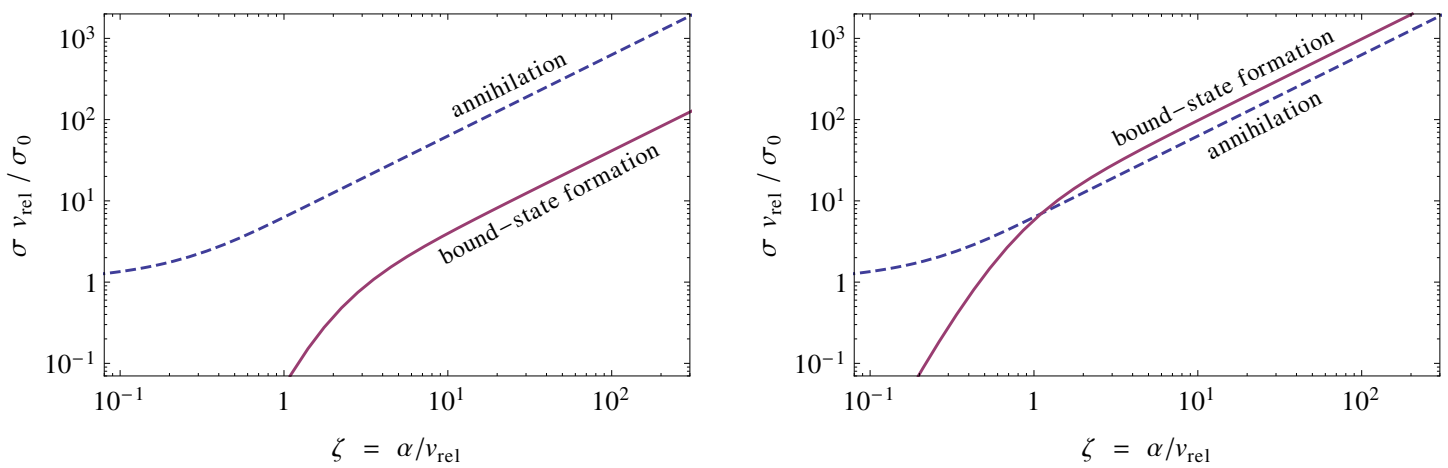

Figure 1. Comparison of the cross-sections for annihilation (dashed blue lines) and the dominant radiative capture process to a bound state (solid purple lines), for a scalar particle-antiparticle pair. The cross-sections times relative velocity, $\sigma v_{\text {rel }}$, are normalised to the perturbative value for $s$-wave annihilation, $\sigma_{0}$. Left: for a scalar mediator, $\sigma_{0}=\pi \alpha^{2} / \mu^{2}$. The leading capture process is to the excited state $\{210\}$ and consists dominantly of the $J=2$ partial wave. At $\zeta \gg 1$, the ratio of the bound-state formation and annihilation cross-sections is $\sigma_{\mathrm{BSF}} / \sigma_{\mathrm{ann}} \simeq 0.066$. Right: for a vector mediator, $\sigma_{0}=\pi \alpha^{2} /\left(2 \mu^{2}\right)$. The leading capture process is to the ground state $\{100\}$ and consists dominantly of the $J=0$ and $J=2$ partial waves. At $\zeta \gg 1, \sigma_{\mathrm{BSF}} / \sigma_{\mathrm{ann}} \simeq 1.56$.

for the decay of unstable bound states into radiation, as well as for the (co-)annihilation of unbound pairs of particles into radiation - two closely related processes. While we lay out our formalism in terms of scalar particles, it is straightforward to extend it to include fermionic species.

We continue by applying our formalism to specific interactions. In section 5 , we consider scalar particles interacting either via a scalar or a vector mediator, and calculate the cross-sections for the dominant radiative capture to a bound state. We estimate the range of validity of our computations, using the unitarity bound on the inelastic cross-section. For bound states made of particle-antiparticle pairs or pairs of self-conjugate particles, we compare BSF with annihilation, and show that BSF can be the dominant inelastic process in the regime where the Sommerfeld effect is important; we sketch this comparison in figure 1. In addition, we calculate the decay rates of particle-antiparticle bound states into force mediators. We cast our results in terms of a minimal parametrisation, which makes their potential implications more transparent, and summarise them in table 1 . We conclude with a discussion of the phenomenological implications of DM bound-state formation in section 6 , and present many of the detailed calculations in the appendices.

The field-theoretic formalism developed in this work has several advantages in comparison to previous quantum mechanical calculations [29, 35, 51, 63, 64]. It can accommodate the possibility of DM coupled to non-Abelian interactions. Such interactions can convert the incoming particles into different species which may subsequently form bound states; importantly, DM coupled to the electroweak interactions of the Standard Model belongs to this category. Moreover, the field-theoretic approach allows for a systematic inclusion of higher-order corrections, both in the interaction strength and in the momentum transfer between the interacting degrees of freedom. 
We emphasise that BSF is distinct from processes such as the direct annihilation into mediators or elastic scattering, in which particles coupled to a long-range interaction may participate. While all these processes are influenced by the Sommerfeld effect, the final-state particles are obviously different. Field-theoretic treatments of the annihilation processes, analogous to the formalism presented here for BSF and discrete level transitions, have been presented in refs. [65, 66].

\section{Bound-state and two-particle state wavefunctions}

We shall consider two scalar particle species $\chi_{1}$ and $\chi_{2}$, interacting via a light or massless force mediator $\varphi$. Specific interaction Lagrangians will be introduced in section 5 (cf. eqs. (5.13), (5.14) and (5.48)). In this section, we aim to determine the wavefunctions which describe two-particle states and bound states with the quantum numbers of $\chi_{1}$ and $\chi_{2}$. Our presentation draws largely from the pedagogical discussions of refs. [67, 68].

Let us first introduce some notation. We denote the masses of $\chi_{1}, \chi_{2}$ and $\varphi$ by $m_{1}, m_{2}$ and $m_{\varphi}$ respectively. We define the total and the reduced $\chi_{1}, \chi_{2}$ masses,

$$
\begin{aligned}
m & \equiv m_{1}+m_{2}, \\
\mu & \equiv \frac{m_{1} m_{2}}{m_{1}+m_{2}} .
\end{aligned}
$$

Obviously, $\mu \leqslant m / 4$. In the following, $\left|\mathcal{B}_{\mathbf{Q}, n}\right\rangle$ stands for a $\chi_{1}-\chi_{2}$ bound state, of total momentum $\mathbf{Q}$ and energy $\omega_{\mathbf{Q}, n}=\sqrt{\mathbf{Q}^{2}+M_{n}^{2}}$, where $n$ denotes collectively all the discrete quantum numbers characterizing the bound state, and $M_{n}<m$ is the bound-state mass. $\left|\mathcal{U}_{\mathbf{Q}, \mathbf{q}}\right\rangle$ stands for a $\chi_{1}-\chi_{2}$ unbound two-particle state, with total momentum $\mathbf{Q}$, expectation value of relative velocity $\mathbf{v}_{\text {rel }}=\mathbf{q} / \mu$, and energy $\omega_{\mathbf{Q}, \mathbf{q}} \geqslant m$. (As is common in scattering theory, we shall often refer to the unbound states as scattering states.) Moreover, $\left|\varphi_{\mathbf{Q}}\right\rangle$ stands for a $\varphi$ particle state with momentum $\mathbf{Q}$ and energy $\omega_{\varphi}=\sqrt{\mathbf{Q}^{2}+m_{\varphi}^{2}}$.

\subsection{The Bethe-Salpeter wavefunctions}

We shall now introduce the Bethe-Salpeter wavefunctions that will appear in the fully relativistic version of the transition amplitudes of sections 3 and 4. The Bethe-Salpeter wavefunctions are related to the more familiar Schrödinger wavefunctions, which we will use in evaluating the amplitudes of interest in the non-relativistic regime.

We are interested in the following wavefunctions:

$$
\begin{aligned}
& \Psi_{\mathbf{Q}, n}\left(x_{1}, x_{2}\right) \equiv\left\langle\Omega\left|T \chi_{1}\left(x_{1}\right) \chi_{2}\left(x_{2}\right)\right| \mathcal{B}_{\mathbf{Q}, n}\right\rangle \\
& \Psi_{\mathbf{Q}, n}^{\star}\left(x_{1}, x_{2}\right) \equiv\left\langle\mathcal{B}_{\mathbf{Q}, n}\left|T \chi_{1}^{\dagger}\left(x_{1}\right) \chi_{2}^{\dagger}\left(x_{2}\right)\right| \Omega\right\rangle
\end{aligned}
$$

and

$$
\begin{aligned}
& \Phi_{\mathbf{Q}, \mathbf{q}}\left(x_{1}, x_{2}\right) \equiv\left\langle\Omega\left|T \chi_{1}\left(x_{1}\right) \chi_{2}\left(x_{2}\right)\right| \mathcal{U}_{\mathbf{Q}, \mathbf{q}}\right\rangle, \\
& \Phi_{\mathbf{Q}, \mathbf{q}}^{\star}\left(x_{1}, x_{2}\right) \equiv\left\langle\mathcal{U}_{\mathbf{Q}, \mathbf{q}}\left|T \chi_{1}^{\dagger}\left(x_{1}\right) \chi_{2}^{\dagger}\left(x_{2}\right)\right| \Omega\right\rangle,
\end{aligned}
$$


where $T$ is the time-ordering operator, and $|\Omega\rangle$ is the vacuum of the interacting theory. If $\chi_{1}, \chi_{2}$ are a particle-antiparticle pair, in the above definitions we replace $\chi_{1} \rightarrow \chi, \chi_{2} \rightarrow \chi^{\dagger}$. Note that * does not denote complex conjugation, for which we shall use the symbol ${ }^{*}$, as usual. In fact

$$
\begin{aligned}
& \Psi_{\mathbf{Q}, n}^{\star}\left(x_{1}, x_{2}\right)=\left\langle\Omega\left|\bar{T} \chi_{1}\left(x_{1}\right) \chi_{2}\left(x_{2}\right)\right| \mathcal{B}_{\mathbf{Q}, n}\right\rangle^{*} \\
& \Phi_{\mathbf{Q}, \mathbf{q}}^{\star}\left(x_{1}, x_{2}\right)=\left\langle\Omega\left|\bar{T} \chi_{1}\left(x_{1}\right) \chi_{2}\left(x_{2}\right)\right| \mathcal{U}_{\mathbf{Q}, \mathbf{q}}\right\rangle^{*}
\end{aligned}
$$

where $\bar{T}$ is the anti-time-ordering operator.

We define the coordinate transformation and its inverse

$$
\begin{aligned}
x & \equiv x_{1}-x_{2}, & X & \equiv \eta_{1} x_{1}+\eta_{2} x_{2}, \\
x_{1} & \equiv X+\eta_{2} x, & x_{2} & \equiv X-\eta_{1} x,
\end{aligned}
$$

where $\eta_{1}+\eta_{2}=1$ for the Jacobian to be 1 , and we choose specifically

$$
\eta_{1,2}=\frac{m_{1,2}}{m_{1}+m_{2}}
$$

In the non-relativistic regime, this choice will enable us to separate the motion of the CM from the relative motion. Using the 4-momentum operator $\hat{P}$, we obtain

$$
\begin{aligned}
& \chi_{1}\left(x_{1}\right)=\exp (i \hat{P} X) \chi_{1}\left(\eta_{2} x\right) \exp (-i \hat{P} X), \\
& \chi_{2}\left(x_{2}\right)=\exp (i \hat{P} X) \chi_{2}\left(-\eta_{1} x\right) \exp (-i \hat{P} X) .
\end{aligned}
$$

Then, the wavefunction of eqs. (2.3) becomes

$$
\begin{aligned}
\Psi_{\mathbf{Q}, n}\left(x_{1}, x_{2}\right)= & \theta\left(x^{0}\right)\left\langle\Omega\left|\chi_{1}\left(x_{1}\right) \chi_{2}\left(x_{2}\right)\right| \mathcal{B}_{\mathbf{Q}, n}\right\rangle+\theta\left(-x^{0}\right)\left\langle\Omega\left|\chi_{2}\left(x_{2}\right) \chi_{1}\left(x_{1}\right)\right| \mathcal{B}_{\mathbf{Q}, n}\right\rangle \\
= & \theta\left(x^{0}\right)\left\langle\Omega\left|e^{i \hat{P} X} \chi_{1}\left(\eta_{2} x\right) \chi_{2}\left(-\eta_{1} x\right) e^{-i \hat{P} X}\right| \mathcal{B}_{\mathbf{Q}, n}\right\rangle \\
& \quad+\theta\left(-x^{0}\right)\left\langle\Omega\left|e^{i \hat{P} X} \chi_{2}\left(-\eta_{1} x\right) \chi_{1}\left(\eta_{2} x\right) e^{-i \hat{P} X}\right| \mathcal{B}_{\mathbf{Q}, n}\right\rangle \\
= & e^{-i Q X}\left\langle\Omega\left|T \chi_{1}\left(\eta_{2} x\right) \chi_{2}\left(-\eta_{1} x\right)\right| \mathcal{B}_{\mathbf{Q}, n}\right\rangle \\
\equiv & e^{-i Q X} \Psi_{\mathbf{Q}, n}(x)
\end{aligned}
$$

where it is understood that $Q^{0}=\omega_{\mathbf{Q}, n}$, and we defined

$$
\Psi_{\mathbf{Q}, n}(x) \equiv\left\langle\Omega\left|T \chi_{1}\left(\eta_{2} x\right) \chi_{2}\left(-\eta_{1} x\right)\right| \mathcal{B}_{\mathbf{Q}, n}\right\rangle
$$

This is the first step in separating the motion of the CM from the relative motion. For notational simplicity, we are using the same symbol for $\Psi_{\mathbf{Q}, n}\left(x_{1}, x_{2}\right)$ and $\Psi_{\mathbf{Q}, n}(x)$. We also define the Fourier transforms

$$
\Psi_{\mathbf{Q}, n}(x) \equiv \int \frac{d^{4} p}{(2 \pi)^{4}} \tilde{\Psi}_{\mathbf{Q}, n}(p) e^{-i p x}, \quad \tilde{\Psi}_{\mathbf{Q}, n}(p) \equiv \int d^{4} x \Psi_{\mathbf{Q}, n}(x) e^{i p x}
$$

We repeat the above for the amplitudes of eqs. (2.4)-(2.6), and summarise the definitions in appendix A. 


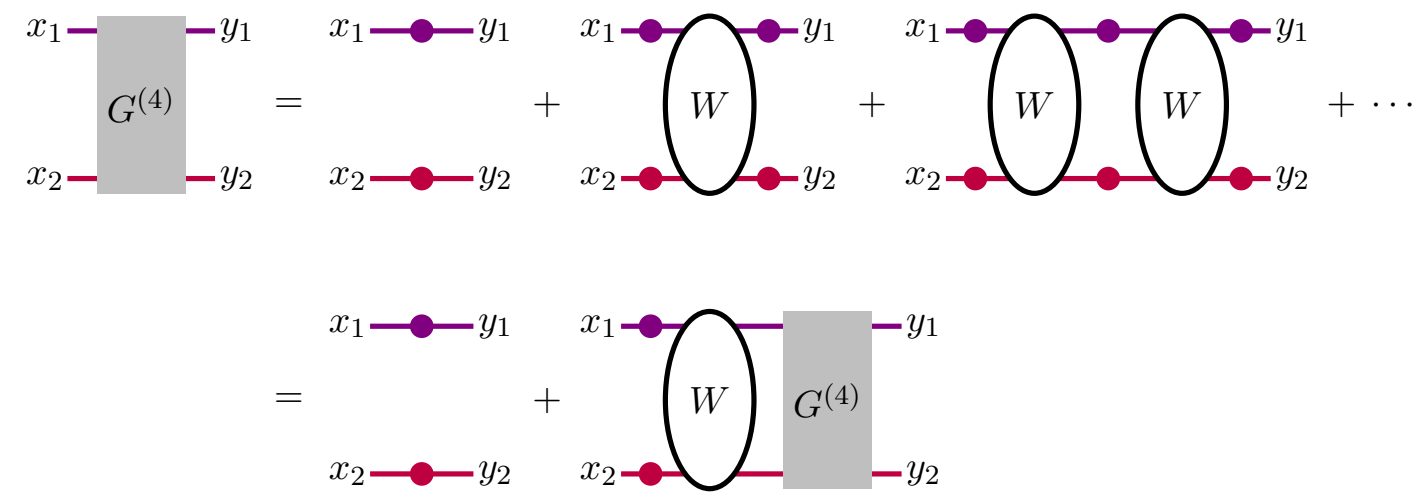

Figure 2. Diagrammatic representation of the Dyson-Schwinger equation (2.19) for the 4-point function $G^{(4)}\left(x_{1}, x_{2}, y_{1}, y_{2}\right)$. $\multimap$ and $\multimap$ stand for the $\chi_{1}$ and $\chi_{2}$ full propagators, respectively.

\subsection{The 4-point Green's function and Dyson-Schwinger equation}

Consider the 4-point Green's function

$$
G^{(4)}\left(x_{1}, x_{2}, y_{1}, y_{2}\right)=\left\langle\Omega\left|T \chi_{1}\left(x_{1}\right) \chi_{2}\left(x_{2}\right) \chi_{1}^{\dagger}\left(y_{1}\right) \chi_{2}^{\dagger}\left(y_{2}\right)\right| \Omega\right\rangle
$$

and let $W\left(x_{1}, x_{2}, y_{1}, y_{2}\right)$ be the perturbative 4-point interaction kernel between $\chi_{1}$ and $\chi_{2}$. Then, $G^{(4)}$ satisfies the Dyson-Schwinger equation,

$$
\begin{aligned}
& G^{(4)}\left(x_{1}, x_{2}, y_{1}, y_{2}\right)= S_{1}\left(x_{1}-y_{1}\right) S_{2}\left(x_{2}-y_{2}\right) \\
&+\int d^{4} z_{1} d^{4} z_{1}^{\prime} d^{4} z_{2} d^{4} z_{2}^{\prime} S_{1}\left(x_{1}-z_{1}\right) S_{2}\left(x_{2}-z_{2}\right) W\left(z_{1}, z_{2} ; z_{1}^{\prime}, z_{2}^{\prime}\right) \\
& \times S_{1}\left(z_{1}^{\prime}-y_{1}\right) S_{2}\left(z_{2}^{\prime}-y_{2}\right)+\ldots,
\end{aligned}
$$

where $S_{1}, S_{2}$ are the full propagators for $\chi_{1}, \chi_{2}$. Symbolically, the above series can be written as

$$
\begin{aligned}
G^{(4)} & =S_{1} S_{2}+S_{1} S_{2} W S_{1} S_{2}+S_{1} S_{2} W S_{1} S_{2} W S_{1} S_{2}+\ldots \\
& =S_{1} S_{2}+S_{1} S_{2} W\left(S_{1} S_{2}+S_{1} S_{2} W S_{1} S_{2}+\ldots\right) \\
& =S_{1} S_{2}+S_{1} S_{2} W G^{(4)}
\end{aligned}
$$

Equation (2.19) is sketched in figure 2 .

Due to translational invariance, $W$ and $G^{(4)}$ depend only on coordinate differences. We shall take them to be $x, y, X-Y$, where we used the definitions of eq. (2.9) and assumed analogous definitions for the $y_{1}, y_{2}$ variables. Thus

$$
\begin{aligned}
G^{(4)}\left(x_{1}, x_{2}, y_{1}, y_{2}\right) & =G^{(4)}(x, y ; X-Y) \\
W\left(x_{1}, x_{2}, y_{1}, y_{2}\right) & =W(x, y ; X-Y)
\end{aligned}
$$


where we retained the same symbols to keep the notation simple. Equation (2.18) becomes

$$
\begin{aligned}
& G^{(4)}(x, y ; X-Y)= S_{1}\left[X-Y+\eta_{2}(x-y)\right] S_{2}\left[X-Y-\eta_{1}(x-y)\right] \\
&+\int d^{4} z d^{4} Z d^{4} z^{\prime} d^{4} Z^{\prime} S_{1}\left[X-Z+\eta_{2}(x-z)\right] S_{2}\left[X-Z-\eta_{1}(x-z)\right] \\
& \times W\left(z, z^{\prime} ; Z-Z^{\prime}\right) G^{(4)}\left(z^{\prime}, y ; Z^{\prime}-Y\right)
\end{aligned}
$$

We define the Fourier transforms of $G, W, S_{1}$ and $S_{2}$,

$$
\begin{gathered}
\tilde{G}^{(4)}\left(p, p^{\prime} ; Q\right) \equiv \int d^{4} x d^{4} y d^{4}(X-Y) G^{(4)}(x, y ; X-Y) \exp \left(i p x-i p^{\prime} y\right) \exp [i Q(X-Y)], \\
\tilde{W}\left(p, p^{\prime} ; Q\right) \equiv \int d^{4} x d^{4} y d^{4}(X-Y) W(x, y ; X-Y) \exp \left(i p x-i p^{\prime} y\right) \exp [i Q(X-Y)]
\end{gathered}
$$

and

$$
\tilde{S}_{j}(p)=\int d^{4} z e^{i p z} S_{j}(z)
$$

with $\tilde{S}_{j}(p)$ being the momentum-space propagator for $\chi_{j}$. From the above, we deduce the relation between the conjugate momenta of $x_{1}, x_{2}$, which we shall call here $p_{1}, p_{2}$, and the conjugate momenta of $x, X$, denoted above as $p, Q$ :

$$
\begin{aligned}
Q & =p_{1}+p_{2}, & p & =\eta_{2} p_{1}-\eta_{1} p_{2}, \\
p_{1} & =\eta_{1} Q+p, & p_{2} & =\eta_{2} Q-p .
\end{aligned}
$$

Analogous relations hold between the conjugate momenta of $y_{1}, y_{2}$ and those of $y, Y$.

For convenience, we also define

$$
S(p ; Q) \equiv \tilde{S}_{1}\left(\eta_{1} Q+p\right) \tilde{S}_{2}\left(\eta_{2} Q-p\right) .
$$

We may now rewrite the Dyson-Schwinger eq. (2.22) for the 4-point function, in momentum space

$$
\tilde{G}^{(4)}\left(p, p^{\prime} ; Q\right)=(2 \pi)^{4} \delta^{4}\left(p-p^{\prime}\right) S(p ; Q)+S(p ; Q) \int \frac{d^{4} k}{(2 \pi)^{4}} \tilde{W}(p, k ; Q) \tilde{G}^{(4)}\left(k, p^{\prime} ; Q\right) .
$$

We shall use eq. (2.29) to derive the Bethe-Salpeter equation for the wavefunctions of section 2.1.

\subsection{Completeness relation and decomposition of the 4-point function}

To compute the Bethe-Salpeter wavefunctions of section 2.1, we have to decompose the 4point Green's function of section 2.2 using the one- and two-particle completeness relation. Then, eq. (2.29) will yield the equations which the wavefunctions $\Psi_{\mathbf{Q}, n}$ and $\Phi_{\mathbf{Q}, \mathbf{q}}$ satisfy.

Including the one- and two-particle states with the same quantum numbers as $\chi_{1}$ and $\chi_{2}$, the completeness relation is

$$
\mathbf{1}=\sum_{n} \int \frac{d^{3} Q}{(2 \pi)^{3} 2 \omega_{\mathbf{Q}, n}}\left|\mathcal{B}_{\mathbf{Q}, n}\right\rangle\left\langle\mathcal{B}_{\mathbf{Q}, n}\left|+\int \frac{d^{3} q}{(2 \pi)^{3}} \frac{d^{3} Q}{(2 \pi)^{3}} \frac{1}{2 \omega_{\mathbf{Q}, \mathbf{q}} 2 \varepsilon_{\mathbf{Q}, \mathbf{q}}}\right| \mathcal{U}_{\mathbf{Q}, \mathbf{q}}\right\rangle\left\langle\mathcal{U}_{\mathbf{Q}, \mathbf{q}}\right|,
$$


where we have assumed the standard relativistic normalization of one-particle momentum eigenstates $\langle\mathbf{p} \mid \mathbf{k}\rangle=2 E_{\mathbf{p}}(2 \pi)^{3} \delta^{3}(\mathbf{p}-\mathbf{k})$, with $E_{\mathbf{p}}$ being the energy of the state $|\mathbf{p}\rangle$. To lowest (zeroth) order in the interaction strength,

$$
2 \omega_{\mathbf{Q}, \mathbf{q}} 2 \varepsilon_{\mathbf{Q}, \mathbf{q}} \simeq 2 E_{1}(\mathbf{q} ; \mathbf{Q}) 2 E_{2}(\mathbf{q} ; \mathbf{Q}),
$$

where

$$
E_{1}(\mathbf{q} ; \mathbf{Q}) \equiv \sqrt{\left(\eta_{1} \mathbf{Q}+\mathbf{q}\right)^{2}+m_{1}^{2}}, \quad E_{2}(\mathbf{q} ; \mathbf{Q}) \equiv \sqrt{\left(\eta_{2} \mathbf{Q}-\mathbf{q}\right)^{2}+m_{2}^{2}} .
$$

Next, we insert the unity operator of eq. (2.30) in $G^{(4)}$, to obtain the decomposition

$$
G^{(4)}(x, y ; X-Y)=\sum_{n} G_{n}^{(4)}(x, y ; X-Y)+G_{\mathcal{U}}^{(4)}(x, y ; X-Y),
$$

where $G_{n}^{(4)}(x, y ; X-Y)$ and $G_{\mathcal{U}}^{(4)}(x, y ; X-Y)$ are the contributions of the bound and the scattering states, respectively. We compute them below. We shall make use of the fact that a non-zero contribution to $G^{(4)}$ from a one- or two-particle state arises only when two annihilation operators act on that state to obtain the quantum numbers of the vacuum. Moreover, in order to extract the poles and the branch-cuts of $G_{n}^{(4)}$ and $G_{\mathcal{U}}^{(4)}$, we will use the integral representation of the $\theta$-function,

$$
\theta(z)=\frac{i}{2 \pi} \int_{-\infty}^{\infty} d k \frac{e^{-i k z}}{k+i \epsilon}
$$

and

$$
\theta\left[\min \left(x_{1}^{0}, x_{2}^{0}\right)-\max \left(y_{1}^{0}, y_{2}^{0}\right)\right]=\theta\left[X^{0}-Y^{0}+h_{-}\left(x^{0}\right)-h_{+}\left(y^{0}\right)\right]
$$

where

$$
h_{ \pm}\left(x^{0}\right) \equiv \frac{1}{2}\left(\eta_{2}-\eta_{1}\right) x^{0} \pm \frac{1}{2}\left|x^{0}\right| .
$$

(For eqs. (2.35) and (2.36), see ref. [68] and appendix B.)

Contribution of the bound states to the 4-point function. The contribution of the $n$th bound state to $G^{(4)}$ is

$$
\begin{aligned}
& G_{n}^{(4)}(x, y ; X-Y)= \\
& =\int \frac{d^{3} K}{(2 \pi)^{3}} \frac{1}{2 \omega_{\mathbf{K}, n}}\left\langle\Omega\left|T \chi_{1}\left(x_{1}\right) \chi_{2}\left(x_{2}\right)\right| \mathcal{B}_{\mathbf{K}, n}\right\rangle\left\langle\mathcal{B}_{\mathbf{K}, n}\left|T \chi_{1}^{\dagger}\left(y_{1}\right) \chi_{2}^{\dagger}\left(y_{2}\right)\right| \Omega\right\rangle \\
& \quad \times \theta\left[\min \left(x_{1}^{0}, x_{2}^{0}\right)-\max \left(y_{1}^{0}, y_{2}^{0}\right)\right] \\
& =\int \frac{d^{3} K}{(2 \pi)^{3}} \frac{1}{2 \omega_{\mathbf{K}, n}} \Psi_{\mathbf{K}, n}(x) \Psi_{\mathbf{K}, n}^{\star}(y) e^{-i \omega_{\mathbf{K}, n}\left(X^{0}-Y^{0}\right)} e^{i \mathbf{K} \cdot(\mathbf{X}-\mathbf{Y})} \theta\left[X^{0}-Y^{0}+h_{-}\left(x^{0}\right)-h_{+}\left(y^{0}\right)\right] \\
& =\int \frac{d^{3} K}{(2 \pi)^{3}} \frac{1}{2 \omega_{\mathbf{K}, n}} \Psi_{\mathbf{K}, n}(x) \Psi_{\mathbf{K}, n}^{\star}(y) e^{-i \omega_{\mathbf{K}, n}\left(X^{0}-Y^{0}\right)} e^{i \mathbf{K} \cdot(\mathbf{X}-\mathbf{Y})} \\
& \quad \times \frac{i}{2 \pi} \int_{-\infty}^{\infty} d K^{0} \frac{\exp \left\{-i\left[K^{0}-\omega_{\mathbf{K}, n}\right]\left[X^{0}-Y^{0}+h_{-}\left(x^{0}\right)-h_{+}\left(y^{0}\right)\right]\right\}}{K^{0}-\omega_{\mathbf{K}, n}+i \epsilon} \\
& =i \int \frac{d^{4} K}{(2 \pi)^{4}} e^{-i K(X-Y)} \Psi_{\mathbf{K}, n}(x) \Psi_{\mathbf{K}, n}^{\star}(y) \frac{\exp \left\{-i\left[K^{0}-\omega_{\mathbf{K}, n}\right]\left[h_{-}\left(x^{0}\right)-h_{+}\left(y^{0}\right)\right]\right\}}{2 \omega_{\mathbf{K}, n}\left[K^{0}-\omega_{\mathbf{K}, n}+i \epsilon\right]},
\end{aligned}
$$


where in the third step we made use of the integral representation of the $\theta$ function, given in eq. (2.34), which introduces the integration over $K^{0}$. The Fourier transform of the above is

$$
\begin{aligned}
\tilde{G}_{n}^{(4)}\left(p, p^{\prime} ; Q\right) & =\int d^{4} x d^{4} y d^{4}(X-Y) e^{i p x-i p^{\prime} y+i Q(X-Y)} G_{n}^{(4)}(x, y ; X-Y) \\
= & i \int d^{4} x d^{4} y e^{i p x-i p^{\prime} y} \Psi_{\mathbf{Q}, n}(x) \Psi_{\mathbf{Q}, n}^{\star}(y) \\
& \times \frac{\exp \left\{-i\left[Q^{0}-\omega_{\mathbf{Q}, n}\right]\left[h_{-}\left(x^{0}\right)-h_{+}\left(y^{0}\right)\right]\right\}}{2 \omega_{\mathbf{Q}, n}\left[Q^{0}-\omega_{\mathbf{Q}, n}+i \epsilon\right]} .
\end{aligned}
$$

At $Q^{0} \rightarrow \omega_{\mathbf{Q}, n}$, this becomes

$$
\tilde{G}_{n}^{(4)}\left(p, p^{\prime} ; Q\right) \rightarrow \frac{i \tilde{\Psi}_{\mathbf{Q}, n}(p) \tilde{\Psi}_{\mathbf{Q}, n}^{\star}\left(p^{\prime}\right)}{2 \omega_{\mathbf{Q}, n}\left[Q^{0}-\omega_{\mathbf{Q}, n}+i \epsilon\right]} .
$$

Equation (2.37) is the contribution of the $n$th bound state to $\tilde{G}^{(4)}\left(p, p^{\prime} ; Q\right)$. Evidently, the scattering amplitude has a pole at energy equal to the bound-state energy.

Contribution of two-particle scattering states to the 4-point function. Following similar steps, we find the contribution of the two-particle states to $G^{(4)}$,

$$
\begin{aligned}
G_{\mathcal{U}}^{(4)}(x, y ; X-Y)=i \int \frac{d^{3} k}{(2 \pi)^{3}} & \frac{d^{4} K}{(2 \pi)^{4}} e^{-i K(X-Y)} \Phi_{\mathbf{K}, \mathbf{k}}(x) \Phi_{\mathbf{K}, \mathbf{k}}^{\star}(y) \\
& \times \frac{\exp \left\{-i\left[K^{0}-\omega_{\mathbf{K}, \mathbf{k}}\right]\left[h_{-}\left(x^{0}\right)-h_{+}\left(y^{0}\right)\right]\right\}}{2 \omega_{\mathbf{K}, \mathbf{k}} 2 \varepsilon_{\mathbf{K}, \mathbf{k}}\left[K^{0}-\omega_{\mathbf{K}, \mathbf{k}}+i \epsilon\right]} .
\end{aligned}
$$

The Fourier transform of $G_{\mathcal{U}}^{(4)}(x, y ; X-Y)$ is

$$
\begin{aligned}
\tilde{G}_{\mathcal{U}}^{(4)}\left(p, p^{\prime} ; Q\right)=i \int \frac{d^{3} q}{(2 \pi)^{3}} \int d^{4} x d^{4} y e^{i p x-i p^{\prime} y} \Phi_{\mathbf{Q}, \mathbf{q}}(x) \Phi_{\mathbf{Q}, \mathbf{q}}^{\star}(y) \\
\times \frac{\exp \left\{-i\left[Q^{0}-\omega_{\mathbf{Q}, \mathbf{q}}\right]\left[h_{-}\left(x^{0}\right)-h_{+}\left(y^{0}\right)\right]\right\}}{2 \omega_{\mathbf{Q}, \mathbf{q}} 2 \varepsilon_{\mathbf{Q}, \mathbf{q}}\left[Q^{0}-\omega_{\mathbf{Q}, \mathbf{q}}+i \epsilon\right]} .
\end{aligned}
$$

Clearly, the contribution of the two-particle states to $\tilde{G}^{(4)}\left(p, p^{\prime} ; Q\right)$ gives rise to a branch-cut in the scattering amplitude.

Summing the contributions from the bound and the scattering states, we obtain the decomposition of the momentum-space 4-point function

$$
\tilde{G}^{(4)}\left(p, p^{\prime} ; Q\right)=\sum_{n} \tilde{G}_{n}^{(4)}\left(p, p^{\prime} ; Q\right)+\tilde{G}_{\mathcal{U}}^{(4)}\left(p, p^{\prime} ; Q\right) .
$$

We shall now combine the Dyson-Schwinger eq. (2.29) and eq. (2.41), to obtain the BetheSalpeter equation for the wavefunctions $\Psi_{\mathbf{Q}, n}$ and $\Phi_{\mathbf{Q}, \mathbf{q}}$.

\subsection{The Bethe-Salpeter equation for bound and scattering states}

We introduce the operator

$$
A\left(p, p^{\prime} ; Q\right) \equiv \frac{(2 \pi)^{4} \delta^{4}\left(p-p^{\prime}\right)}{S(p ; Q)}-\tilde{W}\left(p, p^{\prime} ; Q\right)
$$


Then, the Dyson-Schwinger eq. (2.29) can be cast in the form

$$
\int \frac{d^{4} k}{(2 \pi)^{4}} A(p, k ; Q) \tilde{G}^{(4)}\left(k, p^{\prime} ; Q\right)=(2 \pi)^{4} \delta^{4}\left(p-p^{\prime}\right) .
$$

This is formally solved by

$$
\tilde{G}^{(4)}\left(p, p^{\prime} ; Q\right)=\sum_{n} \frac{1}{c_{n}(Q)} C_{n}(p ; Q) C_{n}^{\dagger}\left(p^{\prime} ; Q\right)+\int \frac{d a}{f_{a}(Q)} F_{a}(p ; Q) F_{a}^{\dagger}\left(p^{\prime} ; Q\right),
$$

where $C_{n}(p ; Q)$ and $F_{a}(p ; Q)$ are the eigenfunctions of the discrete and the continuous spectrum of the operator $A(p, q ; Q)$, with eigenvalues $c_{n}(Q)$ and $f_{a}(Q)$ respectively,

$$
\begin{aligned}
& \int \frac{d^{4} k}{(2 \pi)^{4}} A(p, k ; Q) C_{n}(k ; Q)=c_{n}(Q) C_{n}(p ; Q), \\
& \int \frac{d^{4} k}{(2 \pi)^{4}} A(p, k ; Q) F_{a}(k ; Q)=f_{a}(Q) F_{a}(p ; Q),
\end{aligned}
$$

normalised according to

$$
\sum_{n} C_{n}(p ; Q) C_{n}^{\dagger}\left(p^{\prime} ; Q\right)+\int d a F_{a}(p ; Q) F_{a}^{\dagger}\left(p^{\prime} ; Q\right)=(2 \pi)^{4} \delta^{4}\left(p-p^{\prime}\right) .
$$

We may now collect eqs. (2.37), (2.40), (2.41), and (2.44). Matching the various contributions between (2.41) and (2.44), we deduce the following. For the discrete spectrum:

$$
\begin{aligned}
C_{n}(p ; Q) & \propto \int d^{4} x \Psi_{\mathbf{Q}, n}(x) e^{i p x} e^{-i\left[Q^{0}-\omega_{\mathbf{Q}, n}\right] h_{-}\left(x^{0}\right)}, \\
C_{n}^{\dagger}\left(p^{\prime} ; Q\right) & \propto \int d^{4} y \Psi_{\mathbf{Q}, n}^{\star}(y) e^{-i p^{\prime} y} e^{i\left[Q^{0}-\omega_{\mathbf{Q}, n}\right] h_{+}\left(y^{0}\right)}, \\
c_{n}(Q) & \propto 1-\omega_{\mathbf{Q}, n} / Q^{0} .
\end{aligned}
$$

For the continuous spectrum, we identify $a \rightarrow \mathbf{q}$, and deduce

$$
\begin{aligned}
F_{a}(p ; Q) & \propto \int d^{4} x \Phi_{\mathbf{Q}, \mathbf{q}}(x) e^{i p x} e^{-i\left[Q^{0}-\omega_{\mathbf{Q}, n}\right] h_{-}\left(x^{0}\right)}, \\
F_{a}^{\dagger}\left(p^{\prime} ; Q\right) & \propto \int d^{4} y \Phi_{\mathbf{Q}, \mathbf{q}}^{\star}(x) e^{-i p^{\prime} y} e^{i\left[Q^{0}-\omega_{\mathbf{Q}, n}\right] h_{+}\left(y^{0}\right)}, \\
f_{a}(Q) & \propto 1-\omega_{\mathbf{Q}, \mathbf{q}} / Q^{0} .
\end{aligned}
$$

The relations (2.48), (2.49), (2.51) and (2.52) are stipulated because $c_{n}, f_{a}$ are independent of the momenta $p, p^{\prime}$; all the $p, p^{\prime}$-dependent factors must arise from the eigenfunctions, $C_{n}$ and $F_{a}$. The relations $(2.50),(2.53)$ are warranted so that $C_{n}$ and $F_{a}$ are not singular in the $\operatorname{limit} Q^{0} \rightarrow \omega_{\mathbf{Q}, n}$ and $Q^{0} \rightarrow \omega_{\mathbf{Q}, \mathbf{q}}$ respectively; the factors $\left[1-\omega_{\mathbf{Q}, n} / Q^{0}\right]^{-1},\left[1-\omega_{\mathbf{Q}, \mathbf{q}} / Q^{0}\right]^{-1}$ cannot be part of the eigenfunctions, and thus belong to the eigenvalues.

Inserting the above into the eigenvalue equations (2.45) and (2.46), and taking the limits $Q^{0} \rightarrow \omega_{\mathbf{Q}, n}$ and $Q^{0} \rightarrow \omega_{\mathbf{Q}, \mathbf{q}}$ respectively, we obtain the Bethe-Salpeter equations for 
the bound and the scattering states ${ }^{3}$

$$
\begin{aligned}
& \tilde{\Psi}_{\mathbf{Q}, n}(p)=S(p ; Q) \int \frac{d^{4} k}{(2 \pi)^{4}} \tilde{W}(p, k ; Q) \tilde{\Psi}_{\mathbf{Q}, n}(k), \\
& \tilde{\Phi}_{\mathbf{Q}, \mathbf{q}}(p)=S(p ; Q) \int \frac{d^{4} k}{(2 \pi)^{4}} \tilde{W}(p, k ; Q) \tilde{\Phi}_{\mathbf{Q}, \mathbf{q}}(k) .
\end{aligned}
$$

These are homogeneous equations and do not determine the normalisation of $\Psi_{\mathbf{Q}, n}$ and $\Phi_{\mathbf{Q}, \mathbf{q}}$. Moreover, because we do not know the exact eigenvalues $c_{n}$ and $f_{a}$, we cannot use eq. (2.47) to obtain the normalisation of $\Psi_{\mathbf{Q}, n}$ and $\Phi_{\mathbf{Q}, \mathbf{q}}$. We derive their normalisation in the next section.

\subsection{Normalization of the Bethe-Salpeter wavefunctions}

We derive the normalisation of the wavefunctions $\Psi_{\mathbf{Q}, n}$ and $\Phi_{\mathbf{Q}, \mathbf{q}}$ from the inhomogeneous Dyson-Schwinger eq. (2.29), or equivalently from eq. (2.43), using the method described in ref. [68].

We define the symbolic multiplication

$$
\left[\mathcal{O}_{1} \mathcal{O}_{2}\right]\left(p, p^{\prime} ; Q\right) \equiv \int \frac{d^{4} k}{(2 \pi)^{4}} \mathcal{O}_{1}(p, k ; Q) \mathcal{O}_{2}\left(k, p^{\prime} ; Q\right)
$$

and the unity operator $I\left(p, p^{\prime}\right) \equiv(2 \pi)^{4} \delta^{4}\left(p-p^{\prime}\right)$. Then, eq. (2.43) can be expressed in symbolic form

$$
A \tilde{G}^{(4)}=\tilde{G}^{(4)} A=I .
$$

We differentiate eq. (2.57) over $Q^{0}$ and re-use it, to obtain

$$
\tilde{G}^{(4)} \frac{d A}{d Q^{0}} \tilde{G}^{(4)}=-\frac{d \tilde{G}^{(4)}}{d Q^{0}} .
$$

We shall use eq. (2.58) to obtain the normalisation of the Bethe-Salpeter wavefunctions. For later convenience, we define

$$
\begin{aligned}
& \tilde{N}_{n}\left(p, p^{\prime} ; \mathbf{Q}\right) \equiv i\left[\frac{d A\left(p, p^{\prime} ; Q\right)}{d Q^{0}}\right]_{Q^{0}=\omega_{\mathbf{Q}, n}}, \\
& \tilde{N}_{\mathbf{q}}\left(p, p^{\prime} ; \mathbf{Q}\right) \equiv i\left[\frac{d A\left(p, p^{\prime} ; Q\right)}{d Q^{0}}\right]_{Q^{0}=\omega_{\mathbf{Q}, \mathbf{q}}},
\end{aligned}
$$

and their Fourier transforms,

$$
\begin{aligned}
& N_{n}\left(x, x^{\prime} ; \mathbf{Q}\right) \equiv i\left[\frac{d}{d Q^{0}} \int \frac{d^{4} p}{(2 \pi)^{4}} \frac{d^{4} p^{\prime}}{(2 \pi)^{4}} e^{-i p x} A\left(p, p^{\prime} ; Q\right) e^{i p^{\prime} x^{\prime}}\right]_{Q^{0}=\omega_{\mathbf{Q}, n}}, \\
& N_{\mathbf{q}}\left(x, x^{\prime} ; \mathbf{Q}\right) \equiv i\left[\frac{d}{d Q^{0}} \int \frac{d^{4} p}{(2 \pi)^{4}} \frac{d^{4} p^{\prime}}{(2 \pi)^{4}} e^{-i p x} A\left(p, p^{\prime} ; Q\right) e^{i p^{\prime} x^{\prime}}\right]_{Q^{0}=\omega_{\mathbf{Q}, \mathbf{q}}}
\end{aligned}
$$

\footnotetext{
${ }^{3}$ It is possible to obtain eq. (2.54) more easily, by taking the residue of both sides of eq. (2.41) at $Q^{0} \rightarrow \omega_{\mathbf{Q}, n}$. However, this is not possible for the two-particle states.
} 
Bound states. Substituting the contribution to the 4-point function from the $n$th bound state, given in eq. (2.38), into eq. (2.58), and taking the limit $Q^{0} \rightarrow \omega_{\mathbf{Q}, n}$, we obtain the normalisation condition

$$
\int \frac{d^{4} p}{(2 \pi)^{4}} \frac{d^{4} p^{\prime}}{(2 \pi)^{4}} \tilde{\Psi}_{\mathbf{Q}, n}^{\star}(p) \tilde{N}_{n}\left(p, p^{\prime} ; \mathbf{Q}\right) \tilde{\Psi}_{\mathbf{Q}, n^{\prime}}\left(p^{\prime}\right)=2 \omega_{\mathbf{Q}, n} \delta_{n n^{\prime}} .
$$

In coordinate space, this becomes

$$
\int d^{4} x d^{4} x^{\prime} \Psi_{\mathbf{Q}, n}^{\star}(x) N_{n}\left(x, x^{\prime} ; \mathbf{Q}\right) \Psi_{\mathbf{Q}, n^{\prime}}\left(x^{\prime}\right)=2 \omega_{\mathbf{Q}, n} \delta_{n n^{\prime}} .
$$

Two-particle states. Substituting the contribution to the 4-point function from the two-particle states, eq. (2.40), into eq. (2.58), we deduce the normalisation condition $^{4}$

$$
\int \frac{d^{4} p}{(2 \pi)^{4}} \frac{d^{4} p^{\prime}}{(2 \pi)^{4}} \tilde{\Phi}_{\mathbf{Q}, \mathbf{q}}^{\star}(p) \tilde{N}_{\mathbf{q}}\left(p, p^{\prime} ; \mathbf{Q}\right) \tilde{\Phi}_{\mathbf{Q}, \mathbf{q}^{\prime}}\left(p^{\prime}\right)=2 \omega_{\mathbf{Q}, \mathbf{q}} 2 \varepsilon_{\mathbf{Q}, \mathbf{q}}(2 \pi)^{3} \delta^{3}\left(\mathbf{q}-\mathbf{q}^{\prime}\right) .
$$

In coordinate space, this becomes

$$
\int d^{4} x d^{4} x^{\prime} \Phi_{\mathbf{Q}, \mathbf{q}}^{\star}(x) N_{\mathbf{q}}\left(x, x^{\prime} ; \mathbf{Q}\right) \Phi_{\mathbf{Q}, \mathbf{q}^{\prime}}\left(x^{\prime}\right)=2 \omega_{\mathbf{Q}, \mathbf{q}} 2 \varepsilon_{\mathbf{Q}, \mathbf{q}}(2 \pi)^{3} \delta^{3}\left(\mathbf{q}-\mathbf{q}^{\prime}\right) .
$$

Note that in the fully relativistic case, the normalisation of the wavefunctions depends in general on the potential.

\subsection{The instantaneous approximation and the Schrödinger equation}

In the non-relativistic regime, it is possible to simplify the Bethe-Salpeter equations. The momentum exchange between the two unbound particles is $|\mathbf{q}| \sim \mu v_{\text {rel }}$, while between two bound particles $|\mathbf{q}| \sim \mu \alpha$, with $\alpha$ characterising the interaction strength; in either case, for $\alpha, v_{\text {rel }} \ll 1$, the energy exchange is $q^{0} \sim \mathbf{q}^{2} /(2 \mu) \ll|\mathbf{q}|$. It is then reasonable to ignore the dependence of the kernel $\tilde{W}\left(p, p^{\prime} ; Q\right)$ on $p^{0}, p^{\prime 0}$. This is the instantaneous approximation. ${ }^{5}$ In fact, in the cases of interest, $\tilde{W}\left(p, p^{\prime} ; Q\right)$ depends only on $\left|\mathbf{p}-\mathbf{p}^{\prime}\right|$, rather than on $\mathbf{p}$ and $\mathbf{p}^{\prime}$ separately, and it does not depend on $Q$ (except perhaps for $Q^{2}$, which, in the non-relativistic regime, we shall approximate with $Q^{2} \simeq m^{2}$ ). We shall thus assume that

$$
\tilde{W}\left(p, p^{\prime} ; Q\right) \simeq \mathcal{W}\left(\left|\mathbf{p}-\mathbf{p}^{\prime}\right|\right) .
$$

In this approximation, we deduce from the Bethe-Salpeter eqs. (2.54), (2.55), that $\tilde{\Psi}_{\mathbf{Q}, n}(p) / S(p ; Q)$ and $\tilde{\Phi}_{\mathbf{Q}, \mathbf{q}}(p) / S(p ; Q)$ are independent of $p^{0}$. We define

$$
\begin{aligned}
& \mathcal{S}_{0}(\mathbf{p} ; Q) \equiv \int \frac{d p^{0}}{2 \pi} S(p ; Q), \\
& \tilde{\psi}_{\mathbf{Q}, n}(\mathbf{p}) \equiv \sqrt{2 \mathcal{N}_{\mathbf{Q}}(\mathbf{p})}\left[\frac{\mathcal{S}_{0}(\mathbf{p} ; Q)}{S(p ; Q)}\right] \tilde{\Psi}_{\mathbf{Q}, n}(p), \\
& \tilde{\phi}_{\mathbf{Q}, \mathbf{q}}(\mathbf{p}) \equiv \sqrt{\frac{2 \mathcal{N}_{\mathbf{Q}}(\mathbf{p})}{2 \varepsilon_{\mathbf{Q}, \mathbf{q}}}}\left[\frac{\mathcal{S}_{0}(\mathbf{p} ; Q)}{S(p ; Q)}\right] \tilde{\Phi}_{\mathbf{Q}, \mathbf{q}}(p),
\end{aligned}
$$

\footnotetext{
${ }^{4}$ In fact, from eqs. (2.40) and (2.58) we obtain the normalisation condition described in eq. (2.65) with the functions $\tilde{\Phi}_{\mathbf{Q}, \mathbf{q}}(p)$ replaced by $F_{\mathbf{q}}(p ; Q)=\int d^{4} x e^{i p x} \Phi_{\mathbf{Q}, \mathbf{q}}(x) e^{-i\left[Q^{0}-\omega_{\mathbf{Q}, \mathbf{q}}\right] h\left(x^{0}\right)}$ (cf. eq. (2.51)). Then, taking $Q^{0} \rightarrow \omega_{\mathbf{Q}, \mathbf{q}}$, we obtain the exact form of eq. (2.65).

${ }^{5}$ For a discussion on relativistic corrections, see e.g. ref. [69] and references within.
} 
where we choose the normalization factor

$$
\mathcal{N}_{\mathbf{Q}}(\mathbf{p}) \equiv \frac{E_{1}(\mathbf{p} ; \mathbf{Q}) E_{2}(\mathbf{p} ; \mathbf{Q})}{E_{1}(\mathbf{p} ; \mathbf{Q})+E_{2}(\mathbf{p} ; \mathbf{Q})},
$$

such that we recover the conventional normalisation for $\tilde{\psi}_{\mathbf{Q}, n}$ and $\tilde{\phi}_{\mathbf{Q}, \mathbf{q}}$, as we shall see in section 2.7. We calculate $\mathcal{S}_{0}(\mathbf{p} ; Q)$ in appendix C. Multiplying both sides of eqs. (2.69), (2.70) with $S(p ; Q)$, integrating over $p^{0}$, and using eq. (2.68), it follows that

$$
\begin{aligned}
\tilde{\psi}_{\mathbf{Q}, n}(\mathbf{p}) & =\sqrt{2 \mathcal{N}_{\mathbf{Q}}(\mathbf{p})} \int \frac{d p^{0}}{2 \pi} \tilde{\Psi}_{\mathbf{Q}, n}(p) \\
& =\sqrt{2 \mathcal{N}_{\mathbf{Q}}(\mathbf{p})} \int d^{3} x \Psi_{\mathbf{Q}, n}\left(\left\{x^{0}=0, \mathbf{x}\right\}\right) e^{-i \mathbf{p} \cdot \mathbf{x}}, \\
\tilde{\phi}_{\mathbf{Q}, \mathbf{q}}(\mathbf{p}) & =\sqrt{\frac{2 \mathcal{N}_{\mathbf{Q}}(\mathbf{p})}{2 \varepsilon_{\mathbf{Q}, \mathbf{q}}}} \int \frac{d p^{0}}{2 \pi} \tilde{\Phi}_{\mathbf{Q}, \mathbf{q}}(p) \\
& =\sqrt{\frac{2 \mathcal{N}_{\mathbf{Q}}(\mathbf{p})}{2 \varepsilon_{\mathbf{Q}, \mathbf{q}}}} \int d^{3} x \Phi_{\mathbf{Q}, \mathbf{q}}\left(\left\{x^{0}=0, \mathbf{x}\right\}\right) e^{-i \mathbf{p} \cdot \mathbf{x}} .
\end{aligned}
$$

$\tilde{\psi}_{\mathbf{Q}, n}(\mathbf{p})$ and $\tilde{\phi}_{\mathbf{Q}, \mathbf{q}}(\mathbf{p})$ are sometimes called the "equal-time wavefunctions". From the above, and recalling eqs. (2.7), (2.8) we see that

$$
\tilde{\psi}_{\mathbf{Q}, n}^{\star}(\mathbf{p})=\tilde{\psi}_{\mathbf{Q}, n}^{*}(\mathbf{p}), \quad \tilde{\phi}_{\mathbf{Q}, \mathbf{q}}^{\star}(\mathbf{p})=\tilde{\phi}_{\mathbf{Q}, \mathbf{q}}^{*}(\mathbf{p})
$$

Given the definitions (2.69), (2.70) and eqs. (2.72), (2.73), the Bethe-Salpeter eqs. (2.54) and (2.55) become

$$
\begin{aligned}
& \frac{\tilde{\psi}_{\mathbf{Q}, n}(\mathbf{p})}{\sqrt{2 \mathcal{N}_{\mathbf{Q}}(\mathbf{p})} \mathcal{S}_{0}(\mathbf{p} ; Q)}=\int \frac{d^{3} k}{(2 \pi)^{3}} \frac{\mathcal{W}(|\mathbf{p}-\mathbf{k}|)}{\sqrt{2 \mathcal{N}_{\mathbf{Q}}(\mathbf{k})}} \tilde{\psi}_{\mathbf{Q}, n}(\mathbf{k}), \quad \text { with } Q^{0}=\omega_{\mathbf{Q}, n}, \\
& \frac{\tilde{\phi}_{\mathbf{Q}, \mathbf{q}}(\mathbf{p})}{\sqrt{2 \mathcal{N}_{\mathbf{Q}}(\mathbf{p})} \mathcal{S}_{0}(\mathbf{p} ; Q)}=\int \frac{d^{3} k}{(2 \pi)^{3}} \frac{\mathcal{W}(|\mathbf{p}-\mathbf{k}|)}{\sqrt{2 \mathcal{N}_{\mathbf{Q}}(\mathbf{k})}} \tilde{\phi}_{\mathbf{Q}, \mathbf{q}}(\mathbf{k}), \quad \text { with } Q^{0}=\omega_{\mathbf{Q}, \mathbf{q}} .
\end{aligned}
$$

Non-relativistic approximation. Using the non-relativistic approximation described in appendix C, eqs. (C.18)-(C.22), and setting, in accordance to eq. (C.21),

$$
\begin{aligned}
& \omega_{\mathbf{Q}, n}=m+\mathbf{Q}^{2} / 2 m+\mathcal{E}_{n}, \\
& \omega_{\mathbf{Q}, \mathbf{q}}=m+\mathbf{Q}^{2} / 2 m+\mathcal{E}_{\mathbf{q}},
\end{aligned}
$$

equations (2.75) and (2.76) become

$$
\begin{aligned}
& \left(-\frac{\mathbf{p}^{2}}{2 \mu}+\mathcal{E}_{n}\right) \tilde{\psi}_{n}(\mathbf{p})=-\frac{1}{i 4 m \mu} \int \frac{d^{3} k}{(2 \pi)^{3}} \mathcal{W}(|\mathbf{p}-\mathbf{k}|) \tilde{\psi}_{n}(\mathbf{k}), \\
& \left(-\frac{\mathbf{p}^{2}}{2 \mu}+\mathcal{E}_{\mathbf{q}}\right) \tilde{\phi}_{\mathbf{q}}(\mathbf{p})=-\frac{1}{i 4 m \mu} \int \frac{d^{3} k}{(2 \pi)^{3}} \mathcal{W}(|\mathbf{p}-\mathbf{k}|) \tilde{\phi}_{\mathbf{q}}(\mathbf{k}) .
\end{aligned}
$$

These are the Schrödinger equations for the bound and the scattering states in momentum space. They are eigenvalue equations, and as such, their solutions determine $\mathcal{E}_{n}$ and $\mathcal{E}_{\mathbf{q}}$. 
Because in eqs. (2.79), (2.80), all dependence on the CM momentum $\mathbf{Q}$ has been eliminated, we have dropped this subscript from the $\psi, \phi$ wavefunctions, but kept the same symbols in order to avoid cluttering the notation. Note that from eq. (2.77), it follows that the mass of the bound state is

$$
M_{n}=m+\mathcal{E}_{n} .
$$

It is convenient to Fourier-transform eqs. (2.79) and (2.80) to coordinate space. We set

$$
\begin{aligned}
\psi_{n}(\mathbf{r}) & =\int \frac{d^{3} p}{(2 \pi)^{3}} \tilde{\psi}_{n}(\mathbf{p}) e^{i \mathbf{p} \cdot \mathbf{r}}, & \tilde{\psi}_{n}(\mathbf{p}) & =\int d^{3} r \psi_{n}(\mathbf{r}) e^{-i \mathbf{p} \cdot \mathbf{r}}, \\
\phi_{\mathbf{q}}(\mathbf{r}) & =\int \frac{d^{3} p}{(2 \pi)^{3}} \tilde{\phi}_{\mathbf{q}}(\mathbf{p}) e^{i \mathbf{p} \cdot \mathbf{r}}, & \tilde{\phi}_{\mathbf{q}}(\mathbf{p}) & =\int d^{3} r \phi_{\mathbf{q}}(\mathbf{r}) e^{-i \mathbf{p} \cdot \mathbf{r}} .
\end{aligned}
$$

Acting on both sides of eqs. (2.79), (2.80) with $\int \frac{d^{3} p}{(2 \pi)^{3}} e^{i \mathbf{p} \cdot \mathbf{r}}$, we obtain the Schrödinger equations in coordinate space

$$
\begin{aligned}
& {\left[-\frac{\nabla^{2}}{2 \mu}+V(\mathbf{r})\right] \psi_{n}(\mathbf{r})=\mathcal{E}_{n} \psi_{n}(\mathbf{r}),} \\
& {\left[-\frac{\nabla^{2}}{2 \mu}+V(\mathbf{r})\right] \phi_{\mathbf{q}}(\mathbf{r})=\mathcal{E}_{\mathbf{q}} \phi_{\mathbf{q}}(\mathbf{r}),}
\end{aligned}
$$

where $V(\mathbf{r})$ is the non-relativistic potential,

$$
V(\mathbf{r}) \equiv-\frac{1}{i 4 m \mu} \int \frac{d^{3} k}{(2 \pi)^{3}} \mathcal{W}(\mathbf{k}) e^{i \mathbf{k} \cdot \mathbf{r}} .
$$

We quote the bound-state and scattering-state solutions of the Schrödinger equation for a Coulomb potential, in appendix F, and use them in our computations in section 5 .

\subsection{Normalization of the Schrödinger wavefunctions}

To find the normalization of $\psi_{n}$ and $\phi_{\mathbf{p}}$, it is easiest to follow a similar procedure to that of section 2.5. We first define

$$
\begin{aligned}
\mathcal{G}^{(4)}\left(\mathbf{p}, \mathbf{p}^{\prime} ; Q\right) & \equiv \int \frac{d p^{0}}{2 \pi} \frac{d p^{\prime 0}}{2 \pi} \tilde{G}^{(4)}\left(p, p^{\prime} ; Q\right) \\
\mathcal{G}_{n}^{(4)}\left(\mathbf{p}, \mathbf{p}^{\prime} ; Q\right) & \equiv \int \frac{d p^{0}}{2 \pi} \frac{d p^{\prime 0}}{2 \pi} \tilde{G}_{n}^{(4)}\left(p, p^{\prime} ; Q\right) \\
& =\frac{1}{\sqrt{2 \mathcal{N}_{\mathbf{Q}}(\mathbf{p}) 2 \mathcal{N}_{\mathbf{Q}}\left(\mathbf{p}^{\prime}\right)}} \frac{i \tilde{\psi}_{n}(\mathbf{p}) \tilde{\psi}_{n}^{\star}\left(\mathbf{p}^{\prime}\right)}{2 \omega_{\mathbf{Q}, n}\left[Q^{0}-\omega_{\mathbf{Q}, n}+i \epsilon\right]} \\
\mathcal{G}_{\mathcal{U}}^{(4)}\left(\mathbf{p}, \mathbf{p}^{\prime} ; Q\right) & \equiv \int \frac{d p^{0}}{2 \pi} \frac{d p^{\prime 0}}{2 \pi} \tilde{G}_{\mathcal{U}}^{(4)}\left(p, p^{\prime} ; Q\right) \\
& =\frac{1}{\sqrt{2 \mathcal{N}_{\mathbf{Q}}(\mathbf{p}) 2 \mathcal{N}_{\mathbf{Q}}\left(\mathbf{p}^{\prime}\right)}} \int \frac{d^{3} q}{(2 \pi)^{3}} \frac{i \tilde{\phi}_{\mathbf{q}}(\mathbf{p}) \tilde{\phi}_{\mathbf{q}}^{\star}\left(\mathbf{p}^{\prime}\right)}{2 \omega_{\mathbf{Q}, \mathbf{q}}\left[Q^{0}-\omega_{\mathbf{Q}, \mathbf{q}}+i \epsilon\right]}
\end{aligned}
$$

Integrating eq. (2.29) with respect to $p^{0}, k^{0}$ yields

$$
\mathcal{G}^{(4)}\left(\mathbf{p}, \mathbf{p}^{\prime} ; Q\right)=(2 \pi)^{3} \delta^{3}\left(\mathbf{p}-\mathbf{p}^{\prime}\right) \mathcal{S}_{0}(\mathbf{p} ; Q)+\mathcal{S}_{0}(\mathbf{p} ; Q) \int \frac{d^{3} k}{(2 \pi)^{3}} \mathcal{W}(|\mathbf{p}-\mathbf{k}|) \mathcal{G}^{(4)}\left(\mathbf{k}, \mathbf{p}^{\prime} ; Q\right)
$$


Following the steps of section 2.5, we find the equivalent of eq. (2.58),

$$
\int \frac{d^{3} k}{(2 \pi)^{3}} \mathcal{G}^{(4)}(\mathbf{p}, \mathbf{k} ; Q) \frac{d}{d Q^{0}}\left[\frac{1}{\mathcal{S}_{0}(\mathbf{k} ; Q)}\right] \mathcal{G}^{(4)}\left(\mathbf{k}, \mathbf{p}^{\prime} ; Q\right)=-\frac{d}{d Q^{0}} \mathcal{G}^{(4)}\left(\mathbf{p}, \mathbf{p}^{\prime} ; Q\right) \text {. }
$$

From eq. (C.14),

$$
\frac{d}{d Q^{0}}\left[\frac{1}{\mathcal{S}_{0}(\mathbf{k} ; Q)}\right]=-i 2 \mathcal{N}_{\mathbf{Q}}(\mathbf{k}) 2 Q^{0}
$$

We may now obtain the normalisation conditions for the wavefunctions $\psi_{n}$ and $\phi_{\mathbf{p}}$.

Bound states. Close to the pole, at $Q^{0} \rightarrow \omega_{\mathbf{Q}, n}$, we may substitute the contribution from the $n$th bound state, eq. (2.88), into (2.91). We obtain

$$
\int \frac{d^{3} p}{(2 \pi)^{3}} \tilde{\psi}_{n}^{\star}(\mathbf{p}) \tilde{\psi}_{n}(\mathbf{p})=\int d^{3} r \psi_{n}^{\star}(\mathbf{r}) \psi_{n}(\mathbf{r})=1
$$

Two-particle states. Substituting the contribution from the two-particle states, eq. (2.89) into (2.91), and for $Q^{0} \rightarrow \omega_{\mathbf{Q}, \mathbf{q}}$, we deduce the normalisation condition

$$
\int \frac{d^{3} p}{(2 \pi)^{3}} \tilde{\phi}_{\mathbf{q}}^{\star}(\mathbf{p}) \tilde{\phi}_{\mathbf{q}^{\prime}}(\mathbf{p})=\int d^{3} r \phi_{\mathbf{q}}^{\star}(\mathbf{r}) \phi_{\mathbf{q}^{\prime}}(\mathbf{r})=(2 \pi)^{3} \delta^{3}\left(\mathbf{q}-\mathbf{q}^{\prime}\right) \text {. }
$$

Note that in obtaining the normalisation conditions (2.93) and (2.94), we did not make use of the non-relativistic expansions of the factors $\mathcal{N}_{\mathbf{Q}}(\mathbf{p})$ and $\varepsilon_{\mathbf{Q}, \mathbf{q}}$, given in eqs. (3.33) and (3.35).

\section{Radiative level transitions}

In this section, we determine the amplitudes for the radiative BSF and de-excitation processes

$$
\begin{aligned}
\chi_{1}+\chi_{2} & \rightarrow\left(\chi_{1} \chi_{2}\right)_{\text {bound }}+\varphi \\
\left(\chi_{1} \chi_{2}\right)_{\text {bound }, n^{\prime}} & \rightarrow\left(\chi_{1} \chi_{2}\right)_{\text {bound }, n}+\varphi
\end{aligned}
$$

in terms of the bound-state and scattering-state wavefunctions computed in section 2 and a perturbative interaction which describes the emission of the force mediator. The S-matrix elements of interest are

$$
\begin{aligned}
{ }_{\text {out }}\left\langle\mathcal{B}_{\mathbf{P}, n} ; \varphi_{\mathbf{P}_{\varphi}} \mid \mathcal{U}_{\mathbf{K}, \mathbf{k}}\right\rangle_{\text {in }} & =\left\langle\mathcal{B}_{\mathbf{P}, n} ; \varphi_{\mathbf{P}_{\varphi}}|\mathrm{S}| \mathcal{U}_{\mathbf{K}, \mathbf{k}}\right\rangle, \\
{ }_{\text {out }}\left\langle\mathcal{B}_{\mathbf{P}, n} ; \varphi_{\mathbf{P}_{\varphi}} \mid \mathcal{B}_{\mathbf{K}, n^{\prime}}\right\rangle_{\text {in }} & =\left\langle\mathcal{B}_{\mathbf{P}, n} ; \varphi_{\mathbf{P}_{\varphi}}|\mathrm{S}| \mathcal{B}_{\mathbf{K}, n^{\prime}}\right\rangle
\end{aligned}
$$

where the indices stand for the momenta and the quantum numbers of the corresponding states, as stated in the beginning of section 2 . 


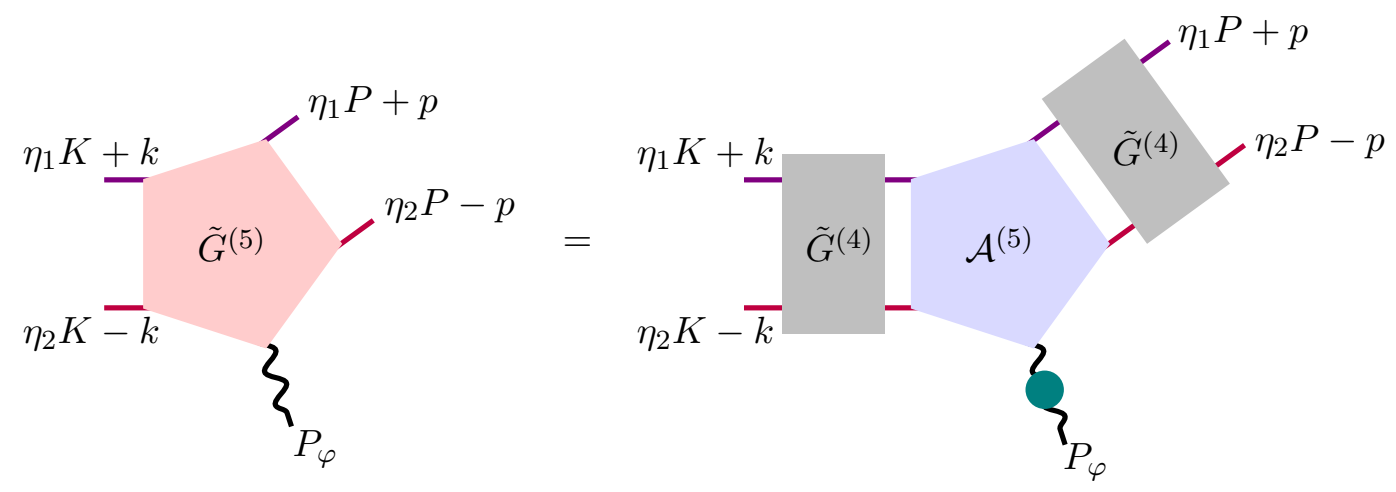

Figure 3. Diagrammatic representation of the equation (3.12) for the 5-point function $\tilde{G}^{(5)}$. $\sim \sim$ stands for the full propagator of the force mediator $\varphi$, which may be either a scalar or a vector boson.

\subsection{The 5-point Green's function}

Since $\mathcal{B}_{\mathbf{Q}, n}$ and $\mathcal{U}_{\mathbf{Q}, \mathbf{q}}$ are generated by the action of $\chi_{1}^{\dagger}$ and $\chi_{2}^{\dagger}$ on the vacuum (cf. eqs. (2.4), (2.6)), in order to compute the S-matrix elements of eqs. (3.3) and (3.4) we need to consider the 5 -point function

$$
G^{(5)}\left(X_{\varphi}, x_{1}, x_{2} ; y_{1}, y_{2}\right) \equiv\left\langle\Omega\left|T \varphi\left(X_{\varphi}\right) \chi_{1}\left(x_{1}\right) \chi_{2}\left(x_{2}\right) \chi_{1}^{\dagger}\left(y_{1}\right) \chi_{2}^{\dagger}\left(y_{2}\right)\right| \Omega\right\rangle
$$

We define the Fourier transform

$$
\begin{aligned}
& \tilde{G}^{(5)}\left(P_{\varphi}, p_{1}, p_{2} ; k_{1}, k_{2}\right)= \\
& \quad=\int d^{4} X_{\varphi} d^{4} x_{1} d^{4} x_{2} d^{4} y_{1} d^{4} y_{2} e^{i\left(P_{\varphi} X_{\varphi}+p_{1} x_{1}+p_{2} x_{2}-k_{1} y_{1}-k_{2} y_{2}\right)} G^{(5)}\left(X_{\varphi}, x_{1}, x_{2} ; y_{1}, y_{2}\right) .
\end{aligned}
$$

As in eq. (2.9), we set

$$
\begin{array}{ll}
x \equiv x_{1}-x_{2}, & X \equiv \eta_{1} x_{1}+\eta_{2} x_{2} \\
y \equiv y_{1}-y_{2}, & Y \equiv \eta_{1} y_{1}+\eta_{2} y_{2},
\end{array}
$$

and rewrite the above as

$$
\begin{aligned}
& \tilde{G}^{(5)}\left(P_{\varphi}, \eta_{1} P+p,\right.\left.\eta_{2} P-p ; \eta_{1} K+k, \eta_{2} K-k\right)= \\
&=\int d^{4} X_{\varphi} d^{4} X d^{4} x d^{4} Y d^{4} y e^{i\left(P_{\varphi} X_{\varphi}+P X+p x-K y-k y\right)} \\
& \quad \times G^{(5)}\left(X_{\varphi}, X+\eta_{2} x, X-\eta_{1} x ; Y+\eta_{2} y, Y-\eta_{1} y\right),
\end{aligned}
$$

i.e. the conjugate momenta of $X, x$ are $P, p$, and the conjugate momenta of $Y, y$ are $K, k$ defined as

$$
\begin{array}{ll}
P \equiv p_{1}+p_{2}, & p \equiv \eta_{2} p_{1}-\eta_{1} p_{2}, \\
K \equiv k_{1}+k_{2}, & k \equiv \eta_{2} k_{1}-\eta_{1} k_{2} .
\end{array}
$$


The 5-point Green's function $G^{(5)}\left(X_{\varphi}, x_{1}, x_{2} ; y_{1}, y_{2}\right)$ is equal to the sum of all connected diagrams with five external points. The momentum-space $\tilde{G}^{(5)}$ is sketched in figure 3 , and can be written as

$$
\begin{aligned}
& \tilde{G}^{(5)}\left(P_{\varphi}, \eta_{1} P+p, \eta_{2} P-p ; \eta_{1} K+k, \eta_{2} K-k\right)= \\
& =\tilde{S}_{\varphi}\left(P_{\varphi}\right) \int \frac{d^{4} p^{\prime}}{(2 \pi)^{4}} \frac{d^{4} k^{\prime}}{(2 \pi)^{4}} \tilde{G}^{(4)}\left(p, p^{\prime} ; P\right)(2 \pi)^{4} \delta^{4}\left(K-P-P_{\varphi}\right) \\
& \quad \times i \mathcal{A}^{(5)}\left(P_{\varphi}, \eta_{1} P+p^{\prime}, \eta_{2} P-p^{\prime} ; \eta_{1} K+k^{\prime}, \eta_{2} K-k^{\prime}\right) \tilde{G}^{(4)}\left(k^{\prime}, k ; K\right),
\end{aligned}
$$

where

$$
\tilde{S}_{\varphi}\left(P_{\varphi}\right)=\frac{i Z_{\varphi}\left(\mathbf{P}_{\varphi}\right)}{P_{\varphi}^{2}-m_{\varphi}^{2}+i \epsilon}
$$

is the $\varphi$ propagator, with

$$
Z_{\varphi}(\mathbf{q}) \equiv\left|\left\langle\Omega|\varphi(0)| \varphi_{\mathbf{q}}\right\rangle\right|^{2}
$$

being the field-strength renormalisation parameter for $\varphi \cdot \mathcal{A}^{(5)}$ is defined via the relation

$$
i \mathcal{C}^{(5)}\left(P_{\varphi}, p_{1}, p_{2} ; k_{1}, k_{2}\right)=\tilde{S}_{\varphi}\left(P_{\varphi}\right) \tilde{S}_{1}\left(p_{1}\right) \tilde{S}_{2}\left(p_{2}\right) \tilde{S}_{1}\left(k_{1}\right) \tilde{S}_{2}\left(k_{2}\right) i \mathcal{A}^{(5)}\left(P_{\varphi}, p_{1}, p_{2} ; k_{1}, k_{2}\right),
$$

where

$$
i \mathcal{C}^{(5)}\left(P_{\varphi}, p_{1}, p_{2} ; k_{1}, k_{2}\right)=\text { sum of all connected diagrams. }
$$

Note that $\mathcal{C}^{(5)}$ may include diagrams that are not fully connected, i.e. diagrams in which external legs are disconnected from each other, ${ }^{6}$ but it does not, of course, include vacuum bubble diagrams. (If only fully connected diagrams contributed to $\mathcal{C}^{(5)}$, then $\mathcal{A}^{(5)}$ would simply be the sum of all connected and amputated diagrams, as conventionally defined.) For later convenience, we also define $\mathcal{C}_{\varphi-\text { amp }}^{(5)}$ as the sum of all connected diagrams with only the $\varphi$-leg amputated,

$$
i \mathcal{C}^{(5)}\left(P_{\varphi}, p_{1}, p_{2} ; k_{1}, k_{2}\right)=\tilde{S}_{\varphi}\left(P_{\varphi}\right) i \mathcal{C}_{\varphi-\mathrm{amp}}^{(5)}\left(P_{\varphi}, p_{1}, p_{2} ; k_{1}, k_{2}\right)
$$

Then, $\mathcal{A}^{(5)}$ appearing in eq. (3.12), becomes

$$
\begin{aligned}
i \mathcal{A}^{(5)}\left(P_{\varphi}, \eta_{1} P+p, \eta_{2} P-p\right. & \left.; \eta_{1} K+k, \eta_{2} K-k\right) \\
& =\frac{i \mathcal{C}_{\varphi-\mathrm{amp}}^{(5)}\left(P_{\varphi}, \eta_{1} P+p, \eta_{2} P-p ; \eta_{1} K+k, \eta_{2} K-k\right)}{S(p ; P) S(k ; K)},
\end{aligned}
$$

where we remind that $S(p ; P) \equiv \tilde{S}_{1}\left(\eta_{1} P+p\right) \tilde{S}_{2}\left(\eta_{2} P-p\right)$ (cf. eq. (2.28)). We sketch eqs. (3.15) and (3.17) in figure 4.

\footnotetext{
${ }^{6}$ In fact, the lowest-order contribution to $\mathcal{C}^{(5)}$, for the transition processes considered in section 5, arises from diagrams that are not fully connected, as shown in figure 7 . However, the entire transition processes are described by fully connected diagrams, shown in figure 8 .
} 

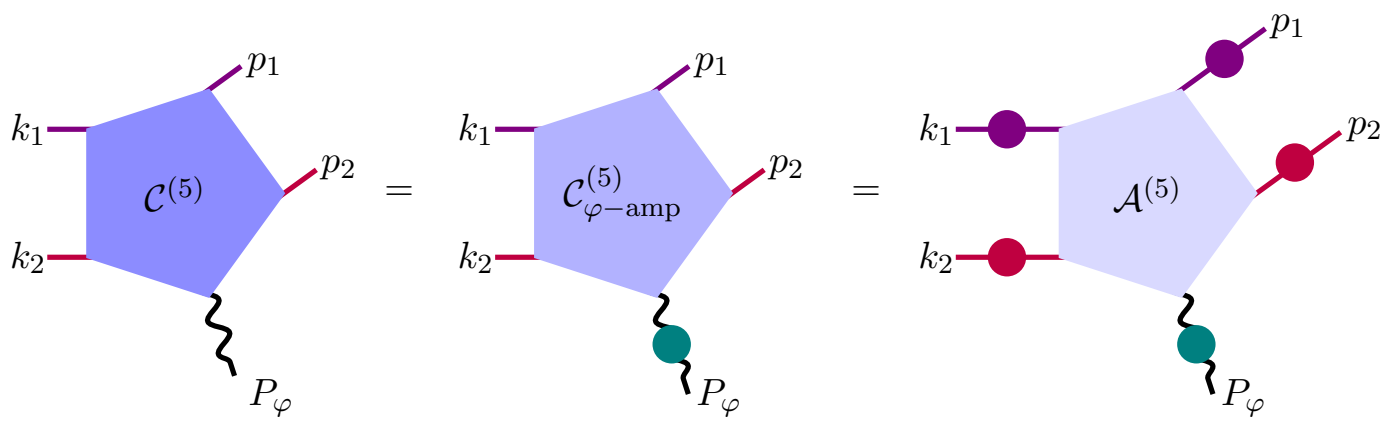

Figure 4. Diagrammatic representation of the equations (3.15) and (3.17). $\mathcal{C}^{(5)}$ stands for the sum of all connected diagrams with no legs amputated; this includes not fully connected diagrams. $\mathcal{C}_{\varphi \text {-amp }}^{(5)}$ is equal to $\mathcal{C}^{(5)}$ with only the $\varphi$-leg amputated. $\longrightarrow$ and - stand for the $\chi_{1}$ and $\chi_{2}$ full propagators, respectively. ح stands for the full propagator of the force mediator $\varphi$, which may be either a scalar or a vector boson.

\subsection{Transition amplitudes}

We now extract the S-matrix elements of eqs. (3.3) and (3.4) from the 5-point Green's function of eq. (3.5). Our analysis follows closely section 7.2 of ref. [70].

Let us first focus on the BSF amplitude of eq. (3.3), for which

$$
P_{\varphi}^{0} \rightarrow \omega_{\varphi}\left(\mathbf{P}_{\varphi}\right), P^{0} \rightarrow \omega_{\mathbf{P}, n}, K^{0} \rightarrow \omega_{\mathbf{K}, \mathbf{k}} .
$$

In this limit, the Lehmann-Symanzik-Zimmermann reduction formula yields

$$
\begin{aligned}
\int d^{4} X_{\varphi} & e^{i P_{\varphi} X_{\varphi}} \int d^{4} X e^{i P X} \int d^{4} Y e^{-i K Y} G^{(5)}\left(X_{\varphi}, X+\eta_{2} x, X-\eta_{1} x ; Y+\eta_{2} y, Y-\eta_{1} y\right) \sim \\
\sim & {\left[\frac{i\left\langle\Omega|\varphi(0)| \varphi_{\mathbf{P}_{\varphi}}\right\rangle}{2 \omega_{\varphi}\left(\mathbf{P}_{\varphi}\right)\left(P_{\varphi}^{0}-\omega_{\varphi}\left(\mathbf{P}_{\varphi}\right)+i \epsilon\right)}\right]\left[\frac{i\left\langle\Omega\left|T \chi_{1}\left(\eta_{2} x\right) \chi_{2}\left(-\eta_{1} x\right)\right| \mathcal{B}_{\mathbf{P}, n}\right\rangle}{2 \omega_{\mathbf{P}, n}\left(P^{0}-\omega_{\mathbf{P}, n}+i \epsilon\right)}\right] } \\
& \times \int \frac{d^{3} k^{\prime}}{(2 \pi)^{3} 2 \varepsilon_{\mathbf{K}, \mathbf{k}^{\prime}}} \frac{i\left\langle\mathcal{U}_{\mathbf{K}, \mathbf{k}^{\prime}}\left|T \chi_{1}^{\dagger}\left(\eta_{2} y\right) \chi_{2}^{\dagger}\left(-\eta_{1} y\right)\right| \Omega\right\rangle}{2 \omega_{\mathbf{K}, \mathbf{k}^{\prime}}\left(K^{0}-\omega_{\mathbf{K}, \mathbf{k}^{\prime}}+i \epsilon\right)}\left\langle\mathcal{B}_{\mathbf{P}, n}, \varphi\left(\mathbf{P}_{\varphi}\right)|S| \mathcal{U}_{\mathbf{K}, \mathbf{k}^{\prime}}\right\rangle .
\end{aligned}
$$

Here, the $\sim$ sign means that the two sides have the same singularities in the limit (3.19); to compute the S-matrix element, we need to extract the residues of these singularities from both sides of eq. (3.20).

In the above expression, the correlation functions involving the $\chi_{1}$ and $\chi_{2}$ fields correspond to the bound and scattering state wavefunctions (cf. eqs. (A.5), (A.8)). The correlation function involving the $\varphi$ field is the $\varphi$ field-strength renormalisation parameter (cf. eq. (3.14)). We Fourier-transform eq. (3.20) with respect to $x, y$, to obtain

$$
\begin{aligned}
& \int d^{4} X_{\varphi} d^{4} X d^{4} Y d^{4} x d^{4} y e^{i\left(P_{\varphi} X_{\varphi}+P X-K Y\right)} e^{i(p x-q y)} G^{(5)}\left(X_{\varphi}, X+\eta_{2} x, X-\eta_{1} x ; Y+\eta_{2} y, Y-\eta_{1} y\right) \\
& \sim\left[\frac{i \sqrt{Z_{\varphi}\left(\mathbf{P}_{\varphi}\right)}}{2 \omega_{\varphi}\left(\mathbf{P}_{\varphi}\right)\left(P_{\varphi}^{0}-\omega_{\varphi}\left(\mathbf{P}_{\varphi}\right)+i \epsilon\right)}\right]\left[\frac{i \tilde{\Psi}_{\mathbf{P}, n}(p)}{2 \omega_{\mathbf{P}, n}\left(P^{0}-\omega_{\mathbf{P}, n}+i \epsilon\right)}\right] \\
& \times \int \frac{d^{3} k^{\prime}}{(2 \pi)^{3} 2 \varepsilon_{\mathbf{K}, \mathbf{k}^{\prime}}} \frac{i \tilde{\Phi}_{\mathbf{K}, \mathbf{k}^{\prime}}^{\star}(q)}{2 \omega_{\mathbf{K}, \mathbf{k}^{\prime}}\left(K^{0}-\omega_{\mathbf{K}, \mathbf{k}^{\prime}}+i \epsilon\right)}\left\langle\mathcal{B}_{\mathbf{P}, n}, \varphi\left(\mathbf{P}_{\varphi}\right)|\mathbf{S}| \mathcal{U}_{\mathbf{K}, \mathbf{k}^{\prime}}\right\rangle . \quad(3.21)
\end{aligned}
$$


The left side of the above equation is $\tilde{G}^{(5)}\left(P_{\varphi}, \eta_{1} P+p, \eta_{2} P-p ; \eta_{1} K+q, \eta_{2} K-q\right)$, which may be decomposed according to eq. (3.12). Recalling eqs. (2.37), (2.40) and (2.41) for the 4-point function $\tilde{G}^{(4)}$, and keeping only the leading singularities in the limit (3.19), the left side of eq. (3.21) becomes

$$
\begin{aligned}
\tilde{G}^{(5)}\left(P_{\varphi}, \eta_{1} P+p, \eta_{2} P-p ; \eta_{1} K+q, \eta_{2} K-q\right) \sim \\
\sim \frac{i Z_{\varphi}\left(\mathbf{P}_{\varphi}\right)}{P_{\varphi}^{2}-m_{\varphi}^{2}+i \epsilon} \int \frac{d^{4} p^{\prime}}{(2 \pi)^{4}} \frac{d^{4} q^{\prime}}{(2 \pi)^{4}} \frac{i \tilde{\Psi}_{\mathbf{P}, n}(p) \tilde{\Psi}_{\mathbf{P}, n}^{\star}\left(p^{\prime}\right)}{2 \omega_{\mathbf{P}, n}\left[P^{0}-\omega_{\mathbf{P}, n}+i \epsilon\right]} \\
\quad \times \int \frac{d^{3} k^{\prime}}{(2 \pi)^{3}} \frac{i \tilde{\Phi}_{\mathbf{K}, \mathbf{k}^{\prime}}\left(q^{\prime}\right) \tilde{\Phi}_{\mathbf{K}, \mathbf{k}^{\prime}}^{\star}(q)}{2 \omega_{\mathbf{K}, \mathbf{k}^{\prime}} 2 \varepsilon_{\mathbf{K}, \mathbf{k}^{\prime}}\left[K^{0}-\omega_{\mathbf{K}, \mathbf{k}^{\prime}}+i \epsilon\right]} \\
\quad \times(2 \pi)^{4} \delta^{4}\left(K-P-P_{\varphi}\right) i \mathcal{A}^{(5)}\left(P_{\varphi}, \eta_{1} P+p^{\prime}, \eta_{2} P-p^{\prime} ; \eta_{1} K+q^{\prime}, \eta_{2} K-q^{\prime}\right) .
\end{aligned}
$$

At $P_{\varphi}^{0} \rightarrow \omega_{\varphi}\left(\mathbf{P}_{\varphi}\right)$ and $P^{0} \rightarrow \omega_{\mathbf{P}, n}$, this expression has the same poles as the right side of eq. (3.21). Identifying their residues, we obtain

$$
\begin{aligned}
& \sqrt{Z_{\varphi}\left(\mathbf{P}_{\varphi}\right)} \int \frac{d^{4} p^{\prime}}{(2 \pi)^{4}} \frac{d^{4} q^{\prime}}{(2 \pi)^{4}} \tilde{\Psi}_{\mathbf{P}, n}^{\star}\left(p^{\prime}\right) \int \frac{d^{3} k^{\prime}}{(2 \pi)^{3}} \frac{i \tilde{\Phi}_{\mathbf{K}, \mathbf{k}^{\prime}}\left(q^{\prime}\right) \tilde{\Phi}_{\mathbf{K}, \mathbf{k}^{\prime}}^{\star}(q)}{2 \omega_{\mathbf{K}, \mathbf{k}^{\prime}} 2 \varepsilon_{\mathbf{K}, \mathbf{k}^{\prime}}\left[K^{0}-\omega_{\mathbf{K}, \mathbf{k}^{\prime}}+i \epsilon\right]} \\
& \times(2 \pi)^{4} \delta^{4}\left(K-P-P_{\varphi}\right) i \mathcal{A}^{(5)}\left(P_{\varphi}, \eta_{1} P+p^{\prime}, \eta_{2} P-p^{\prime} ; \eta_{1} K+q^{\prime}, \eta_{2} K-q^{\prime}\right) \sim \\
& \sim \int \frac{d^{3} k^{\prime}}{(2 \pi)^{3} 2 \varepsilon_{\mathbf{K}, \mathbf{k}^{\prime}}} \frac{i \tilde{\Phi}_{\mathbf{K}, \mathbf{k}^{\prime}}^{\star}(q)}{2 \omega_{\mathbf{K}, \mathbf{k}^{\prime}}\left[K^{0}-\omega_{\mathbf{K}, \mathbf{k}^{\prime}}+i \epsilon\right]}\left\langle\mathcal{B}_{\mathbf{P}, n}, \varphi\left(\mathbf{P}_{\varphi}\right)|\mathbf{S}| \mathcal{U}_{\mathbf{K}, \mathbf{k}^{\prime}}\right\rangle .
\end{aligned}
$$

We still have to extract the leading singularity at $K^{0} \rightarrow \omega_{\mathbf{K}, \mathbf{k}}$. We multiply both sides of the above expression with $\tilde{N}_{\mathbf{k}}\left(q, q^{\prime \prime} ; \mathbf{K}\right) \Phi_{\mathbf{K}, \mathbf{k}}\left(q^{\prime \prime}\right)$, integrate over $q$ and $q^{\prime \prime}$, and use the orthonormality condition (2.65), to obtain the S-matrix element for BSF

$$
\begin{aligned}
&\left\langle\mathcal{B}_{\mathbf{P}, n} ;\right.\left.\varphi_{\mathbf{P}_{\varphi}}|\mathrm{S}| \mathcal{U}_{\mathbf{K}, \mathbf{k}}\right\rangle=\sqrt{Z_{\varphi}\left(\mathbf{P}_{\varphi}\right)} \int \frac{d^{4} p}{(2 \pi)^{4}} \frac{d^{4} q}{(2 \pi)^{4}} \tilde{\Psi}_{\mathbf{P}, n}^{\star}(p) \tilde{\Phi}_{\mathbf{K}, \mathbf{k}}(q) \\
& \times(2 \pi)^{4} \delta^{4}\left(K-P-P_{\varphi}\right) i \mathcal{A}^{(5)}\left(P_{\varphi}, \eta_{1} P+p, \eta_{2} P-p ; \eta_{1} K+q, \eta_{2} K-q\right) .
\end{aligned}
$$

Following similar steps, we obtain the S-matrix element for transition between discrete energy levels, $\left\langle\mathcal{B}_{\mathbf{P}, n} ; \varphi_{\mathbf{P}_{\varphi}}|\mathrm{S}| \mathcal{B}_{\mathbf{K}, n^{\prime}}\right\rangle$.

In standard notation, we write the S-matrix elements as

$$
\begin{aligned}
\left\langle\mathcal{B}_{\mathbf{P}, n} ; \varphi_{\mathbf{P}_{\varphi}}|\mathrm{S}| \mathcal{U}_{\mathbf{K}, \mathbf{k}}\right\rangle & =(2 \pi)^{4} \delta^{4}\left(K-P-P_{\varphi}\right) i \mathcal{M}_{\mathbf{k} \rightarrow n}, \\
\left\langle\mathcal{B}_{\mathbf{P}, n} ; \varphi_{\mathbf{P}_{\varphi}}|\mathrm{S}| \mathcal{B}_{\mathbf{K}, n^{\prime}}\right\rangle & =(2 \pi)^{4} \delta^{4}\left(K-P-P_{\varphi}\right) i \mathcal{M}_{n^{\prime} \rightarrow n},
\end{aligned}
$$

with

$$
\begin{aligned}
\mathcal{M}_{\mathbf{k} \rightarrow n}=\sqrt{Z_{\varphi}\left(\mathbf{P}_{\varphi}\right)} & \int \frac{d^{4} p}{(2 \pi)^{4}} \frac{d^{4} q}{(2 \pi)^{4}} \tilde{\Psi}_{\mathbf{P}, n}^{\star}(p) \tilde{\Phi}_{\mathbf{K}, \mathbf{k}}(q) \\
& \times \mathcal{A}^{(5)}\left(P_{\varphi}, \eta_{1} P+p, \eta_{2} P-p ; \eta_{1} K+q, \eta_{2} K-q\right), \\
\mathcal{M}_{n^{\prime} \rightarrow n}=\sqrt{Z_{\varphi}\left(\mathbf{P}_{\varphi}\right)} & \int \frac{d^{4} p}{(2 \pi)^{4}} \frac{d^{4} q}{(2 \pi)^{4}} \tilde{\Psi}_{\mathbf{P}, n}^{\star}(p) \tilde{\Psi}_{\mathbf{K}, n^{\prime}}(q) \\
& \times \mathcal{A}^{(5)}\left(P_{\varphi}, \eta_{1} P+p, \eta_{2} P-p ; \eta_{1} K+q, \eta_{2} K-q\right) .
\end{aligned}
$$


If non-fully connected diagrams contribute to the perturbative part of the transition amplitudes, then $\mathcal{A}^{(5)}$ should be replaced by $\mathcal{C}_{\varphi-\text { amp }}^{(5)}$ using eq. (3.18). In this case, we obtain

$$
\begin{aligned}
\mathcal{M}_{\mathbf{k} \rightarrow n}=\sqrt{Z_{\varphi}\left(\mathbf{P}_{\varphi}\right)} \int \frac{d^{4} p}{(2 \pi)^{4}} \frac{d^{4} q}{(2 \pi)^{4}} \frac{\tilde{\Psi}_{\mathbf{P}, n}^{\star}(p)}{S(p ; P)} \frac{\tilde{\Phi}_{\mathbf{K}, \mathbf{k}}(q)}{S(q ; K)} \\
\quad \times \mathcal{C}_{\varphi-\mathrm{amp}}^{(5)}\left(P_{\varphi}, \eta_{1} P+p, \eta_{2} P-p ; \eta_{1} K+q, \eta_{2} K-q\right) \\
\mathcal{M}_{n^{\prime} \rightarrow n}=\sqrt{Z_{\varphi}\left(\mathbf{P}_{\varphi}\right)} \int \frac{d^{4} p}{(2 \pi)^{4}} \frac{d^{4} q}{(2 \pi)^{4}} \frac{\tilde{\Psi}_{\mathbf{P}, n}^{\star}(p)}{S(p ; P)} \frac{\tilde{\Psi}_{\mathbf{K}, n^{\prime}}(q)}{S(q ; K)} \\
\quad \times \mathcal{C}_{\varphi-\mathrm{amp}}^{(5)}\left(P_{\varphi}, \eta_{1} P+p, \eta_{2} P-p ; \eta_{1} K+q, \eta_{2} K-q\right) .
\end{aligned}
$$

In the case of a vector mediator $\varphi_{\mu}, Z_{\varphi}$ becomes the charge-renormalisation parameter and the amplitudes contain the polarisation vector $\epsilon^{\mu}$, i.e. $\mathcal{A}^{(5)}=\epsilon^{\mu} \mathcal{A}_{\mu}^{(5)}$ and $\mathcal{C}_{\varphi-\text { amp }}^{(5)}=$ $\epsilon^{\mu} \mathcal{C}_{\varphi-\mathrm{amp}, \mu}^{(5)}$.

\subsection{Instantaneous approximation}

In the instantaneous and non-relativistic approximations, we may express the transition amplitudes in terms of the Schrödinger wavefunctions defined in eqs. (2.69), (2.70), as follows

$$
\begin{aligned}
& \mathcal{M}_{\mathbf{k} \rightarrow n} \simeq \sqrt{2 \varepsilon_{\mathbf{K}, \mathbf{k}}} \int \frac{d^{3} p}{(2 \pi)^{3}} \frac{d^{3} q}{(2 \pi)^{3}} \frac{\tilde{\psi}_{n}^{\star}(\mathbf{p}) \tilde{\phi}_{\mathbf{k}}(\mathbf{q})}{\sqrt{2 \mathcal{N}_{\mathbf{P}}(\mathbf{p}) 2 \mathcal{N}_{\mathbf{K}}(\mathbf{q})}} \mathcal{M}_{\text {trans }}(\mathbf{q} ; \mathbf{p}), \\
& \mathcal{M}_{n^{\prime} \rightarrow n} \simeq \int \frac{d^{3} p}{(2 \pi)^{3}} \frac{d^{3} q}{(2 \pi)^{3}} \frac{\tilde{\psi}_{n}^{\star}(\mathbf{p}) \tilde{\psi}_{n^{\prime}}(\mathbf{q})}{\sqrt{2 \mathcal{N}_{\mathbf{P}}(\mathbf{p}) 2 \mathcal{N}_{\mathbf{K}}(\mathbf{q})}} \mathcal{M}_{\text {trans }}(\mathbf{q} ; \mathbf{p}),
\end{aligned}
$$

where we took $Z_{\varphi}\left(\mathbf{P}_{\varphi}\right) \simeq 1$ to lowest order, and set

$\mathcal{M}_{\text {trans }}(\mathbf{q} ; \mathbf{p}) \equiv \frac{1}{\mathcal{S}_{0}(\mathbf{q} ; K) \mathcal{S}_{0}(\mathbf{p} ; P)} \int \frac{d p^{0}}{2 \pi} \frac{d q^{0}}{2 \pi} \mathcal{C}_{\varphi-\text { amp }}^{(5)}\left(P_{\varphi}, \eta_{1} P+p, \eta_{2} P-p ; \eta_{1} K+q, \eta_{2} K-q\right)$

It is sufficient for our purposes, and consistent with our approximation (see footnote 14 ), to expand the normalisation factors up to first order in $\mathbf{p}^{2}, \mathbf{q}^{2}$, as follows

$$
\begin{aligned}
& \mathcal{N}_{\mathbf{Q}}(\mathbf{p}) \simeq \mu\left[1+\frac{\mathbf{p}^{2}}{2 \mu^{2}}\left(1-\frac{3 \mu}{m}\right)\right], \\
& \frac{1}{\sqrt{2 \mathcal{N}_{\mathbf{P}}(\mathbf{p}) 2 \mathcal{N}_{\mathbf{K}}(\mathbf{q})}} \simeq \frac{1}{2 \mu}\left[1-\frac{\mathbf{p}^{2}+\mathbf{q}^{2}}{4 \mu^{2}}\left(1-\frac{3 \mu}{m}\right)\right] .
\end{aligned}
$$

The $\mathbf{p}^{2}, \mathbf{q}^{2}$ terms in eq. (3.34) introduce corrections of order $\alpha^{2}$ and $v_{\text {rel }}^{2}$ (see appendix F), where $\alpha$ parametrises the strength of the interaction (for a Coulomb potential, it is the fine-structure constant) and gives the expectation value of the relative velocity inside the bound state. Similar corrections arise also in $\mathcal{M}_{\text {trans }}$ (see appendix E). We shall retain such corrections only where the dominant term in the respective expansion vanishes, as 
in the case of degenerate particles interacting via scalar boson exchange (see section 5.1). Moreover, from eqs. (2.31) and (2.78), we find that to zeroth order in the relative velocity,

$$
\varepsilon_{\mathbf{K}, \mathbf{k}} \simeq \mu .
$$

Because $\varepsilon_{\mathbf{K}, \mathbf{k}}$ factors out of the integrals, as seen in eq. (3.30), we neglect $\mathbf{k}^{2}$ corrections, which always produce subdominant terms in $v_{\text {rell }}^{2}$. In addition, in our computations, we consistently ignore corrections that involve at least one power of the total momentum of any of the initial or final states (denoted typically with capital letters). In the CM frame, these momenta are of order $\sim \mathcal{O}\left(\alpha^{2}+v_{\text {rel }}^{2}\right)$, which renders their scalar products with any other momenta, of higher order in $\alpha$ and $v_{\text {rel }}$ than the $\mathbf{p}^{2}, \mathbf{q}^{2}$ corrections.

We shall employ eqs. (3.30)-(3.34) to evaluate the transition amplitudes of section 5 .

\subsection{On-shell approximation}

Let us now consider the case when the perturbative part of the transition amplitude $\mathcal{C}^{(5)}$ consists only of fully connected diagrams. ${ }^{7}$ Then, $\mathcal{A}^{(5)}\left(P_{\varphi}, \eta_{1} P+p, \eta_{2} P-p ; \eta_{1} K+q, \eta_{2} K-\right.$ $q$ ) is the perturbative amplitude for the $2 \rightarrow 3$ transition (with no on-shell conditions imposed). Equation (3.32) becomes

$$
\begin{aligned}
\mathcal{M}_{\text {trans }}(\mathbf{q} ; \mathbf{p}) \simeq \int \frac{d p^{0}}{2 \pi} \frac{d q^{0}}{2 \pi} \frac{S(p ; P) S(q ; K)}{\mathcal{S}_{0}(\mathbf{p} ; P) \mathcal{S}_{0}(\mathbf{q} ; K)} & \\
& \times \mathcal{A}^{(5)}\left(P_{\varphi}, \eta_{1} P+p, \eta_{2} P-p ; \eta_{1} K+q, \eta_{2} K-q\right) .
\end{aligned}
$$

Provided that $\mathcal{A}^{(5)}$ has no singularities in $p^{0}$ and $q^{0,8}$ the integrations over $p^{0}, q^{0}$ force the evaluation of $\mathcal{A}^{(5)}$ on the poles of $S(p ; P)$ and $S(q ; K)$ that are located in either the lower or upper $p^{0}$ and $q^{0}$ complex planes (depending on the choice of integration contours). As described in appendix $\mathrm{C}$, each integration picks out two poles: one physical pole, which corresponds to setting one of the particles on-shell, and one unphysical pole, where the energy of the other particle is negative (cf. eqs. (C.11), (C.12)). In the non-relativistic regime, the contribution from the physical pole dominates. For concreteness, let us take these poles to be in the lower $p^{0}, q^{0}$ complex planes (as in eq. (C.12)),

$$
\begin{aligned}
& p^{0}=-\eta_{1} P^{0}+E_{1}(\mathbf{p} ; \mathbf{P})-i \epsilon, \\
& q^{0}=-\eta_{1} K^{0}+E_{1}(\mathbf{q} ; \mathbf{K})-i \epsilon .
\end{aligned}
$$

Fixing $p^{0}$ and $q^{0}$ to the pole values means that the energies of the $\chi_{1}, \chi_{2}$ particles in the bound state and in the two-particle states are specified as functions of the 3-momenta $\mathbf{p}, \mathbf{q}, \mathbf{P}, \mathbf{K}$ and the quantum numbers $n$ and $\mathbf{k}$ (note that $P^{0}=\omega_{\mathbf{P}, n}$ and $K^{0}=\omega_{\mathbf{K}, \mathbf{k}}$ ), as

\footnotetext{
${ }^{7}$ This is the case if the initial-state particles and the particles participating in the bound state are different.

${ }^{8} \mathcal{A}^{(5)}\left(P_{\varphi}, \eta_{1} P+p, \eta_{2} P-p ; \eta_{1} K+q, \eta_{2} K-q\right)$ may have singularities in $p^{0}$ and $q^{0}$ (for the energy of interest, $\left.K^{0}=\omega_{\mathbf{K}, \mathbf{k}}\right)$, if the initial-state particles and the particles participating in the bound state are different.
} 
follows

$$
\begin{array}{ll}
p_{1}^{0}=\eta_{1} P^{0}+p^{0}=E_{1}(\mathbf{p} ; \mathbf{P}), & p_{2}^{0}=\omega_{\mathbf{P}, n}-E_{1}(\mathbf{p} ; \mathbf{P}) \simeq E_{2}(\mathbf{p} ; \mathbf{P})+\mathcal{E}_{n}-\mathbf{p}^{2} /(2 \mu), \\
q_{1}^{0}=\eta_{1} K^{0}+q^{0}=E_{1}(\mathbf{q} ; \mathbf{K}), & q_{2}^{0}=\omega_{\mathbf{K}, \mathbf{k}}-E_{1}(\mathbf{q} ; \mathbf{K}) \simeq E_{2}(\mathbf{q} ; \mathbf{K})+\mathcal{E}_{\mathbf{k}}-\mathbf{q}^{2} /(2 \mu),
\end{array}
$$

where we used eqs. (2.77), (2.78) and (C.18), (C.19). Evidently, in both the bound state and the two-particle state, the $\chi_{2}$ degree of freedom is off-shell, by $\mathcal{E}_{n}-\mathbf{p}^{2} / 2 \mu$ and $\mathcal{E}_{\mathbf{k}}-\mathbf{q}^{2} / 2 \mu$ respectively. However, $\left\langle\mathbf{p}^{2}\right\rangle /(2 \mu) \sim-\mathcal{E}_{n}$ and $\left\langle\mathbf{q}^{2}\right\rangle /(2 \mu) \sim \mathcal{E}_{\mathbf{k}}$; provided that $\mathcal{E}_{n}, \mathcal{E}_{\mathbf{k}} \ll \mu{ }^{9}$ we may ignore this small deviation from the on-shell condition and evaluate $\mathcal{A}^{(5)}$ on-shell. Then, from eq. (3.32), we obtain

$$
\mathcal{M}_{\text {trans }}(\mathbf{q} ; \mathbf{p}) \simeq\left[\mathcal{A}^{(5)}\left(P_{\varphi}, \eta_{1} P+p, \eta_{2} P-p ; \eta_{1} K+q, \eta_{2} K-q\right)\right]_{\text {on-shell }} .
$$

This is the approximation presented in section 5.3 of ref. [70]. Note that, for consistency, when using eq. (3.39) inside eqs. (3.30) and (3.31), the normalisation factor of eq. (3.34) should be approximated to zeroth order in $\mathbf{p}^{2}, \mathbf{q}^{2}$. Indeed, corrections of the order $\mathbf{p}^{2}, \mathbf{q}^{2}$ arise not only due to the normalisation factor, but also due to the off-shellness of the amplitude $\mathcal{A}^{(5)}$. When important, such corrections should be included self-consistently, by making use both of the full expansion of eq. (3.34), and of the off-shell momenta of eqs. (3.37), (3.38) instead of the on-shell conditions.

We will not make use of eq. (3.39) in our computations in section 5.

\subsection{Bound-state formation cross-sections}

In the $\mathrm{CM}$ frame $(\mathbf{K}=0)$, the differential cross-section for radiative $\mathrm{BSF}, \mathcal{U}_{\mathbf{K}=0, \mathbf{k}} \rightarrow$ $\mathcal{B}_{\mathbf{P}, n}+\varphi_{-\mathbf{P}}$, is

$$
\frac{d \sigma_{\mathrm{BSF}}^{(n)}}{d \Omega}=\frac{1}{2 \sqrt{\left(s-m_{1}^{2}-m_{2}^{2}\right)^{2}-4 m_{1}^{2} m_{2}^{2}}} \frac{|\mathbf{P}|}{16 \pi^{2} \sqrt{s}}\left|\mathcal{M}_{\mathbf{k} \rightarrow n}\right|^{2},
$$

where $s=\omega_{\mathbf{K}=0, \mathbf{k}}^{2} \simeq\left(m+\mathcal{E}_{\mathbf{k}}\right)^{2}$ (cf. eq. (2.78)), and $\mathcal{M}_{\mathbf{k} \rightarrow n}$ is found from eq. (3.30). The bound-state and mediator momenta are

$$
|\mathbf{P}|=\left[\frac{\left(s-M_{n}^{2}-m_{\varphi}^{2}\right)^{2}-4 M_{n}^{2} m_{\varphi}^{2}}{4 s}\right]^{1 / 2} \simeq\left(\mathcal{E}_{\mathbf{k}}-\mathcal{E}_{n}\right)\left[1-\frac{m_{\varphi}^{2}}{\left(\mathcal{E}_{\mathbf{k}}-\mathcal{E}_{n}\right)^{2}}\right]^{1 / 2}
$$

where we used $M_{n}=m+\mathcal{E}_{n}$ (cf. eq. (2.81)). In addition,

$$
2 \sqrt{\left(s-m_{1}^{2}-m_{2}^{2}\right)^{2}-4 m_{1}^{2} m_{2}^{2}} \simeq 4 m k=4 m \mu v_{\text {rel }} .
$$

Then

$$
\frac{d \sigma_{\mathrm{BSF}}^{(n)}}{d \Omega}=\frac{\left(\mathcal{E}_{\mathbf{k}}-\mathcal{E}_{n}\right)}{64 \pi^{2} m^{2} \mu v_{\mathrm{rel}}}\left[1-\frac{m_{\varphi}^{2}}{\left(\mathcal{E}_{\mathbf{k}}-\mathcal{E}_{n}\right)^{2}}\right]^{1 / 2}\left|\mathcal{M}_{\mathbf{k} \rightarrow n}\right|^{2} .
$$

\footnotetext{
${ }^{9}$ For a Coulomb or Yukawa potential with fine structure constant $\alpha$, these conditions are equivalent to $\alpha, v_{\text {rel }} \ll 1$ (cf. eq. (5.4)).
} 
Partial-wave decomposition and unitarity. It will be useful to decompose the amplitude $\mathcal{M}_{\mathbf{k} \rightarrow n}$ in partial waves

$$
\mathcal{M}_{\mathbf{k} \rightarrow n}(\Omega)=\sum_{J}\left(\frac{2 J+1}{4 \pi}\right) \mathcal{M}_{J} P_{J}(\cos \theta)
$$

where $P_{J}$ are the Legendre polynomials, and

$$
\mathcal{M}_{J}=\int d \Omega P_{J}(\cos \theta) \mathcal{M}_{\mathbf{k} \rightarrow n}(\Omega)
$$

Then, eq. (3.43) gives

$$
\sigma_{\mathrm{BSF}}^{(n)}=\sum_{J} \sigma_{\mathrm{BSF}, J}^{(n)} .
$$

with the partial-wave cross-section given by

$$
\sigma_{\mathrm{BSF}, J}^{(n)}=\frac{\left(\mathcal{E}_{\mathbf{k}}-\mathcal{E}_{n}\right)}{64 \pi^{2} m^{2} \mu v_{\mathrm{rel}}}\left[1-\frac{m_{\varphi}^{2}}{\left(\mathcal{E}_{k}-\mathcal{E}_{n}\right)^{2}}\right]^{1 / 2} \frac{2 J+1}{4 \pi}\left|\mathcal{M}_{J}\right|^{2} .
$$

Unitarity implies an upper limit on the partial-wave inelastic cross-sections. In the non-relativistic regime, for the $J$ th partial wave, this is [71]

$$
\sigma_{\text {inel }, J} \leqslant\left(\sigma_{\text {inel }, J}\right)_{\max }=\frac{(2 J+1) \pi}{\mu^{2} v_{\text {rel }}^{2}} .
$$

For a given inelastic process and associated cross-section, this bound yields an estimate for the value of the coupling at which the probability for inelastic scattering saturates. In section 5 , we use the unitarity bound to deduce the range of validity of our calculation.

\subsection{Bound-state de-excitation rates}

The differential rate for the radiative de-excitation of a bound state, $\mathcal{B}_{\mathbf{K}=0, n^{\prime}} \rightarrow \mathcal{B}_{\mathbf{P}, n}+$ $\varphi_{-\mathbf{P}}$, is

$$
\frac{d \Gamma_{n^{\prime} \rightarrow n}}{d \Omega}=\frac{|\mathbf{P}|}{32 \pi^{2} M_{n^{\prime}}^{2}}\left|\mathcal{M}_{n^{\prime} \rightarrow n}\right|^{2}
$$

where $\left|\mathcal{M}_{n^{\prime} \rightarrow n}\right|^{2}$ is found from eq. (3.31) and $|\mathbf{P}|$ is given by eq. (3.41) with the replacement $\mathcal{E}_{\mathbf{k}} \rightarrow \mathcal{E}_{n^{\prime}}$. Setting $M_{n}^{\prime}=m+\mathcal{E}_{n} \simeq m$, we obtain

$$
\Gamma_{n^{\prime} \rightarrow n} \simeq \frac{\left(\mathcal{E}_{n^{\prime}}-\mathcal{E}_{n}\right)}{32 \pi^{2} m^{2}}\left[1-\frac{m_{\varphi}^{2}}{\left(\mathcal{E}_{n^{\prime}}-\mathcal{E}_{n}\right)^{2}}\right]^{1 / 2} \int\left|\mathcal{M}_{n^{\prime} \rightarrow n}\right|^{2} d \Omega .
$$

\section{Decay of unstable bound states and (co-)annihilation of unbound pairs}

\subsection{Non-perturbative amplitude}

If an unbound $\chi_{1}, \chi_{2}$ pair can (co-)annihilate into a number of light particles $f_{1}, \cdots f_{N}$, then the $\chi_{1}-\chi_{2}$ bound states are unstable against decay into the same final states (provided that this is allowed by angular momentum conservation). For example, unbound and bound 


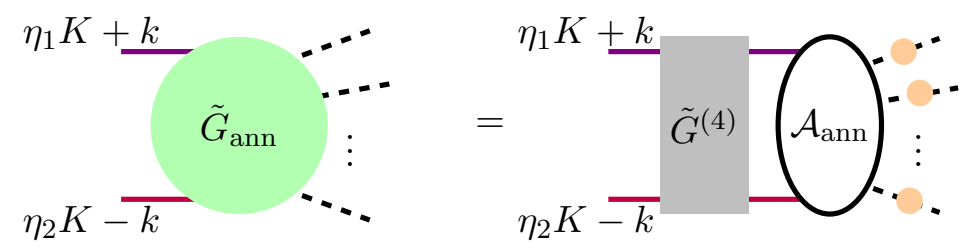

Figure 5. Diagrammatic representation of equation (4.5). - - - represent the full propagators of annihilation/decay products $f_{j}$.

particle-antiparticle pairs can annihilate and decay, respectively, into force mediators. (Co)annihilation and bound-state decay have the same diagrammatic representation, shown in figure 5 ; the difference in evaluating these two processes is which initial state is singled out from the $G^{(4)}$ function. In this section, we express the (co-)annihilation and the bound-state decay amplitudes,

$$
\begin{aligned}
{ }_{\text {out }}\left\langle f_{1} f_{2} \cdots f_{N} \mid \mathcal{U}_{\mathbf{K}, \mathbf{k}}\right\rangle_{\text {in }} & =\left\langle f_{1} f_{2} \cdots f_{N}|\mathrm{~S}| \mathcal{U}_{\mathbf{K}, \mathbf{k}}\right\rangle, \\
\text { out }\left\langle f_{1} f_{2} \cdots f_{N} \mid \mathcal{B}_{\mathbf{K}, n}\right\rangle_{\text {in }} & =\left\langle f_{1} f_{2} \cdots f_{N}|\mathrm{~S}| \mathcal{B}_{\mathbf{K}, n}\right\rangle
\end{aligned}
$$

in terms of the initial state wavefunction and the perturbative interaction that gives rise to these processes. ${ }^{10}$

To calculate the S-matrix elements (4.1) and (4.2), we need to consider the Green's function

$$
G_{\mathrm{ann}}\left(x_{1}, x_{2}, \ldots x_{N} ; y_{1}, y_{2}\right) \equiv\left\langle\Omega\left|T f\left(x_{1}\right) f\left(x_{2}\right) \ldots f\left(x_{N}\right) \chi_{1}^{\dagger}\left(y_{1}\right) \chi_{2}^{\dagger}\left(y_{2}\right)\right| \Omega\right\rangle .
$$

and its Fourier transform

$$
\begin{aligned}
\tilde{G}_{\mathrm{ann}}\left(p_{1}, \ldots p_{N} ; k_{1}, k_{2}\right)= & \prod_{j=1}^{N} \int d^{4} x_{j} e^{i p_{j} x_{j}} \\
& \times \int d^{4} y_{1} d^{4} y_{2} e^{-i\left(k_{1} y_{1}+k_{2} y_{2}\right)} G_{\mathrm{ann}}\left(x_{1}, \cdots, x_{N} ; y_{1}, y_{2}\right) .
\end{aligned}
$$

Let $\mathcal{A}_{\text {ann }}\left(p_{1}, \cdots p_{N} ; k_{1}, k_{2}\right)$ be the sum of all connected and amputated diagrams contributing the (co-)annihilation of a $\chi_{1}, \chi_{2}$ pair with momenta $k_{1}, k_{2}$, into $f_{1}, \cdots, f_{N}$ particles with momenta $p_{1}, \cdots, p_{N}{ }^{11}$ Then, as sketched in figure 5 ,

$$
\begin{aligned}
& \tilde{G}_{\mathrm{ann}}(\left.p_{1}, \cdots, p_{N} ; \eta_{1} K+k, \eta_{2} K-k\right)= \\
& \quad=\prod_{j=1}^{N} \tilde{S}_{f_{j}}\left(p_{j}\right) \int \frac{d^{4} k^{\prime}}{(2 \pi)^{4}} \mathcal{A}_{\mathrm{ann}}\left(p_{1}, \cdots, p_{N} ; \eta_{1} K+k^{\prime}, \eta_{2} K-k^{\prime}\right) \tilde{G}^{(4)}\left(k^{\prime}, k ; K\right),
\end{aligned}
$$

\footnotetext{
${ }^{10}$ As already mentioned, for annihilation processes, similar analyses have been carried out in previous works, e.g. [40-42, 65, 66, 72-74].

${ }^{11}$ Note that, in contrast to level transitions processes, all diagrams contributing to the perturbative part of the (co-)annihilation processes are fully connected. The amputation of fully connected diagrams is well defined, and it is thus sensible to express the full amplitudes for the (co-)annihilation and decay processes of interest, in terms of the sum of amputated diagrams.
} 
where $\tilde{S}_{f_{j}}\left(p_{j}\right)$ is the propagator of the $f_{j}$ particle with momentum $p_{j}$. As always, energymomentum conservation implies that

$$
\mathcal{A}_{\text {ann }}\left(p_{1}, \cdots, p_{N} ; k_{1}, k_{2}\right)=i(2 \pi)^{4} \delta^{4}\left(k_{1}+k_{2}-p_{1} \cdots-p_{N}\right) \mathcal{M}_{\mathrm{ann}}^{\mathrm{pert}}\left(k_{1}, k_{2} ; p_{1}, \cdots, p_{N}\right),
$$

where $\mathcal{M}_{\text {ann }}^{\text {pert }}$ is the perturbative annihilation amplitude, with no on-shell conditions imposed on the incoming and outgoing degrees of freedom. We follow a similar procedure as in section 3.2 , and determine the S-matrix elements of interest to be

$$
\begin{aligned}
& \left\langle f_{1} \cdots f_{N}|\mathrm{~S}| \mathcal{U}_{\mathbf{K}, \mathbf{k}}\right\rangle=(2 \pi)^{4} \delta^{4}\left(K-p_{1} \cdots-p_{N}\right) i \mathcal{M}_{\mathrm{ann}} \\
& \left\langle f_{1} \cdots f_{N}|\mathrm{~S}| \mathcal{B}_{\mathbf{K}, n}\right\rangle=(2 \pi)^{4} \delta^{4}\left(K-p_{1} \cdots-p_{N}\right) i \mathcal{M}_{\mathrm{dec}}^{(n)}
\end{aligned}
$$

with

$$
\begin{aligned}
\mathcal{M}_{\mathrm{ann}} & =\prod_{j=1}^{N} \sqrt{Z_{f_{j}}\left(\mathbf{p}_{j}\right)} \int \frac{d^{4} q}{(2 \pi)^{4}} \tilde{\Phi}_{\mathbf{K}, \mathbf{k}}(q) \mathcal{M}_{\mathrm{ann}}^{\mathrm{pert}}\left(\eta_{1} K+q, \eta_{2} K-q ; p_{1}, \cdots, p_{N}\right) \\
& \simeq \sqrt{2 \varepsilon_{\mathbf{K}, \mathbf{k}}} \int \frac{d^{3} q}{(2 \pi)^{3}} \frac{\tilde{\phi}_{\mathbf{k}}(\mathbf{q})}{\sqrt{2 \mathcal{N}_{\mathbf{K}}(\mathbf{q})}} \int \frac{d q^{0}}{2 \pi} \frac{S(q ; K)}{\mathcal{S}_{0}(\mathbf{q} ; K)} \mathcal{M}_{\mathrm{ann}}^{\mathrm{pert}}\left(\eta_{1} K+q, \eta_{2} K-q ; p_{1}, \cdots, p_{N}\right) \\
\mathcal{M}_{\mathrm{dec}}^{(n)} & =\prod_{j=1}^{N} \sqrt{Z_{f_{j}}\left(\mathbf{p}_{j}\right)} \int \frac{d^{4} q}{(2 \pi)^{4}} \tilde{\Psi}_{\mathbf{K}, n}(q) \mathcal{M}_{\mathrm{ann}}^{\mathrm{pert}}\left(\eta_{1} K+q, \eta_{2} K-q ; p_{1}, \cdots, p_{N}\right) \\
& \simeq \int \frac{d^{3} q}{(2 \pi)^{3}} \frac{\tilde{\psi}_{n}(\mathbf{q})}{\sqrt{2 \mathcal{N}_{\mathbf{K}}(\mathbf{q})}} \int \frac{d q^{0}}{2 \pi} \frac{S(q ; K)}{\mathcal{S}_{0}(\mathbf{q} ; K)} \mathcal{M}_{\mathrm{ann}}^{\text {pert }}\left(\eta_{1} K+q, \eta_{2} K-q ; p_{1}, \cdots, p_{N}\right)
\end{aligned}
$$

where $K^{0}=\omega_{\mathbf{K}, \mathbf{k}}$ and $K^{0}=\omega_{\mathbf{K}, n}$, respectively. In the above, $Z_{f_{j}}(\mathbf{p}) \equiv\left|\left\langle\Omega\left|f_{j}(0)\right| f_{j, \mathbf{p}}\right\rangle\right|^{2}$, with $f_{j, \mathbf{p}}$ being a $f_{j}$ particle with momentum p. In the second line eqs. (4.9) and (4.10), we have used the instantaneous approximation for the wavefunctions, and set $Z_{f_{j}}(\mathbf{p}) \simeq 1$.

\subsection{On-shell approximation}

Following the same arguments as in section 3.3, we may evaluate the perturbative amplitude $\mathcal{M}_{\text {ann }}^{\text {pert }}$ on-shell. This enables us to express the annihilation and decay amplitudes as follows

$$
\begin{aligned}
\mathcal{M}_{\mathrm{ann}} & \simeq \int \frac{d^{3} q}{(2 \pi)^{3}} \tilde{\phi}_{\mathbf{k}}(\mathbf{q}) \hat{\mathcal{M}}_{\mathrm{ann}}^{\mathrm{pert}}\left(\eta_{1} \mathbf{K}+\mathbf{q}, \eta_{2} \mathbf{K}-\mathbf{q} ; \mathbf{p}_{1}, \cdots, \mathbf{p}_{N}\right), \\
\mathcal{M}_{\mathrm{dec}}^{(n)} & \simeq \frac{1}{\sqrt{2 \mu}} \int \frac{d^{3} q}{(2 \pi)^{3}} \tilde{\psi}_{n}(\mathbf{q}) \hat{\mathcal{M}}_{\mathrm{ann}}^{\text {pert }}\left(\eta_{1} \mathbf{K}+\mathbf{q}, \eta_{2} \mathbf{K}-\mathbf{q} ; \mathbf{p}_{1}, \cdots, \mathbf{p}_{N}\right),
\end{aligned}
$$

where $\hat{\mathcal{M}}_{\text {ann }}^{\text {pert }}$ is the on-shell perturbative (co-)annihilation amplitude. Note that, as discussed below eq. (3.39), the integrands in eqs. (4.11), (4.12) admit $\mathbf{q}^{2}$ and higher order corrections from the normalisation factor of eq. (3.33) and from the off-shellness of the perturbative amplitude $\mathcal{M}_{\text {ann }}^{\text {pert }}$.

\subsection{Two-body (co-)annihilation cross-sections and bound-state decay rates}

Let us now focus on the case of decays and (co-)annihilations into two final-state particles. In the CM frame $(\mathbf{K}=0)$, the momenta of the final particles are $\left|\mathbf{p}_{1}\right|=\left|\mathbf{p}_{2}\right|=|\mathbf{p}|$, with 
$|\mathbf{p}| \simeq \omega_{\mathbf{K}=0, \mathbf{k}} / 2=\left(m+\mathcal{E}_{\mathbf{k}}\right) / 2 \simeq m / 2$ in the case of (co-)annihilation, and $|\mathbf{p}| \simeq M_{n} / 2=$ $\left(m+\mathcal{E}_{n}\right) / 2 \simeq m / 2$ in the case of decay. We ignore the masses of the final-state particles for simplicity. The (co-)annihilation and decay amplitudes can be expanded in partial waves as follows

$$
\begin{aligned}
& \mathcal{M}_{\mathrm{ann}}\left(\Omega_{\mathbf{p}}\right)=\sum_{\ell=0}^{\infty}\left(\frac{2 \ell+1}{4 \pi}\right) P_{\ell}\left(\cos \theta_{\mathbf{p}}\right) \mathcal{M}_{\mathrm{ann}, \ell} \\
& \mathcal{M}_{\mathrm{dec}}^{(n)}\left(\Omega_{\mathbf{p}}\right)=\sum_{\ell=0}^{\infty}\left(\frac{2 \ell+1}{4 \pi}\right) P_{\ell}\left(\cos \theta_{\mathbf{p}}\right) \mathcal{M}_{\mathrm{dec}, \ell}^{(n)}
\end{aligned}
$$

where

$$
\begin{aligned}
\mathcal{M}_{\mathrm{ann}, \ell} & \equiv \int d \Omega_{\mathbf{p}} P_{\ell}\left(\cos \theta_{\mathbf{p}}\right) \mathcal{M}_{\mathrm{ann}}\left(\Omega_{\mathbf{p}}\right), \\
\mathcal{M}_{\mathrm{dec}, \ell}^{(n)} & \equiv \int d \Omega_{\mathbf{p}} P_{\ell}\left(\cos \theta_{\mathbf{p}}\right) \mathcal{M}_{\mathrm{dec}}^{(n)}\left(\Omega_{\mathbf{p}}\right) .
\end{aligned}
$$

Here and in the following, the indices in the angle variables specify the vector to which this angle refers; a double index denotes the angle between the two vectors. The (co)annihilation cross-section times relative velocity and the decay rate are

$$
\begin{aligned}
\sigma_{\mathrm{ann}} v_{\mathrm{rel}} & =\frac{f_{s}}{128 \pi^{2} m \mu} \int\left|\mathcal{M}_{\mathrm{ann}}\left(\Omega_{\mathbf{p}}\right)\right|^{2} d \Omega_{\mathbf{p}}=\frac{f_{s}}{128 \pi^{2} m \mu} \sum_{\ell} \frac{2 \ell+1}{4 \pi}\left|\mathcal{M}_{\mathrm{ann}, \ell}\right|^{2} \\
\Gamma_{\mathrm{dec}}^{(n)} & =\frac{f_{s}}{64 \pi^{2} m} \int\left|\mathcal{M}_{\mathrm{dec}}^{(n)}\left(\Omega_{\mathbf{p}}\right)\right|^{2} d \Omega_{\mathbf{p}}=\frac{f_{s}}{64 \pi^{2} m} \sum_{\ell} \frac{2 \ell+1}{4 \pi}\left|\mathcal{M}_{\mathrm{dec}, \ell}^{(n)}\right|^{2}
\end{aligned}
$$

where $f_{s}=1 / 2$ if the final-state particles are identical, or $f_{s}=1$ otherwise. We shall now express $\mathcal{M}_{\mathrm{ann}, \ell}$ and $\mathcal{M}_{\mathrm{dec}, \ell}^{(n)}$ in terms of the perturbative on-shell annihilation amplitude.

We expand $\hat{\mathcal{M}}_{\text {ann }}^{\text {pert }}$ in partial waves, as follows

$$
\hat{\mathcal{M}}_{\mathrm{ann}}^{\text {pert }}(\mathbf{q},-\mathbf{q} ; \mathbf{p},-\mathbf{p})=\sum_{\ell=0}^{\infty} \frac{\tilde{a}_{\ell}}{(m \mu)^{\ell}}|\mathbf{p}|^{\ell}|\mathbf{q}|^{\ell} P_{\ell}\left(\cos \theta_{\mathbf{q}, \mathbf{p}}\right) .
$$

In general, the expansion coefficients $\tilde{a}_{J}$ may depend on $\mathbf{q}$; in the non-relativistic regime, they can be expanded as

$$
\tilde{a}_{\ell}(\mathbf{q}) \simeq a_{\ell}+\mathcal{F}_{\ell}\left(\mathbf{q}^{2}, \boldsymbol{\epsilon}_{A} \cdot \mathbf{q}\right)
$$

where $\boldsymbol{\epsilon}_{A}$ stands for the polarisation vectors of possible final-state vector bosons, and $\mathcal{F}_{\ell}$ is a polynomial function of the scalar products $\mathbf{q}^{2}$ and $\boldsymbol{\epsilon}_{A} \cdot \mathbf{q}$ that vanishes at $\mathbf{q}=0$. Note that $|\mathbf{p}|$ is determined by energy conservation. $a_{\ell}$ and $\mathcal{F}_{\ell}$ may depend on scalar products such as $\mathbf{p}^{2}, \boldsymbol{\epsilon}_{A} \cdot \mathbf{p}$ and $\boldsymbol{\epsilon}_{A} \cdot \boldsymbol{\epsilon}_{B}$. In the following, we consider only the $a_{\ell}$ contribution to $\tilde{a}_{\ell}$; any corrections arising from the q-dependent terms of eq. (4.20) may be included only in conjunction with similar corrections arising from the normalisation factor of eq. (3.33) and from the off-shellness of the perturbative amplitude $\mathcal{M}_{\text {ann }}^{\text {pert }}$. 
We may now insert eq. (4.19) into eqs. (4.11) and (4.12), and use the formula

$$
\begin{aligned}
\int d \Omega_{\mathbf{p}} P_{\ell^{\prime}}\left(\cos \theta_{\mathbf{p}}\right) \int \frac{d^{3} q}{(2 \pi)^{3}} & \tilde{\phi}_{\mathbf{k}}(\mathbf{q})|\mathbf{q}|^{\ell} P_{\ell}\left(\cos \theta_{\mathbf{q}, \mathbf{p}}\right)= \\
& =\delta_{\ell \ell^{\prime}} \frac{(2 \ell+1) ! !}{i^{\ell}(2 \ell+1) \ell !}\left[\frac{d^{\ell}}{d r^{\ell}} \int d \Omega_{\mathbf{r}} P_{\ell}\left(\cos \theta_{\mathbf{r}}\right) \phi_{\mathbf{k}}(\mathbf{r})\right]_{\mathbf{r}=0},
\end{aligned}
$$

and similarly for $\psi_{n}$. We prove eq. (4.21) in appendix D. Keeping only the q-independent term from the expansion of eq. (4.20), we find

$$
\begin{aligned}
& \mathcal{M}_{\mathrm{ann}, \ell} \simeq \frac{a_{\ell}|\mathbf{p}|^{\ell}}{(m \mu)^{\ell}} \frac{(2 \ell+1) ! !}{i^{\ell}(2 \ell+1) \ell !}\left[\frac{d^{\ell}}{d r^{\ell}} \int d \Omega_{\mathbf{r}} P_{\ell}\left(\cos \theta_{\mathbf{r}}\right) \phi_{\mathbf{k}}(\mathbf{r})\right]_{\mathbf{r}=0}, \\
& \mathcal{M}_{\mathrm{dec}, \ell}^{(n)} \simeq \frac{a_{\ell}|\mathbf{p}|^{\ell}}{\sqrt{2 \mu}(m \mu)^{\ell}} \frac{(2 \ell+1) ! !}{i^{\ell}(2 \ell+1) \ell !}\left[\frac{d^{\ell}}{d r^{\ell}} \int d \Omega_{\mathbf{r}} P_{\ell}\left(\cos \theta_{\mathbf{r}}\right) \psi_{n}(\mathbf{r})\right]_{\mathbf{r}=0} .
\end{aligned}
$$

Using eqs. (4.17) and (4.18), we find the contribution of the $\ell$ partial wave to $\sigma_{\text {ann }} v_{\text {rel }}$ and $\Gamma_{\text {dec }}^{(n)}$ to be

$$
\begin{aligned}
\left(\sigma_{\mathrm{ann}} v_{\mathrm{rel}}\right)_{\ell} & =\sigma_{\ell} S_{\ell, \mathrm{ann}}, \\
\Gamma_{\ell, \mathrm{dec}}^{(n)} & =\sigma_{\ell} S_{\ell, \mathrm{dec}}
\end{aligned}
$$

where

$$
\sigma_{\ell}=\frac{[\ell ! /(2 \ell) ! !]^{2}}{2 \ell+1} \frac{f_{s}\left|a_{\ell}\right|^{2}}{32 \pi m \mu}
$$

and

$$
\begin{aligned}
S_{\ell, \mathrm{ann}} & =\frac{\left[(2 \ell+1) ! /(\ell !)^{2}\right]^{2}}{4^{\ell+2} \pi^{2} \mu^{2 \ell}}\left|\frac{d^{\ell}}{d r^{\ell}} \int d \Omega_{\mathbf{r}} P_{\ell}\left(\cos \theta_{\mathbf{r}}\right) \phi_{\mathbf{k}}(\mathbf{r})\right|_{\mathbf{r}=0}^{2} \\
S_{\ell, \mathrm{dec}}^{(n)} & =\frac{\left[(2 \ell+1) ! /(\ell !)^{2}\right]^{2}}{4^{\ell+2} \pi^{2} \mu^{2 \ell}}\left|\frac{d^{\ell}}{d r^{\ell}} \int d \Omega_{\mathbf{r}} P_{\ell}\left(\cos \theta_{\mathbf{r}}\right) \psi_{n}(\mathbf{r})\right|_{\mathbf{r}=0}^{2}
\end{aligned}
$$

In the limit where the interaction in the two-particle state can be neglected, $\phi_{\mathbf{k}}(\mathbf{r})=e^{i \mathbf{k} \cdot \mathbf{r}}$ and $S_{\ell, \text { ann }}=(|\mathbf{k}| / \mu)^{2 \ell}=v_{\text {rel }}^{2 \ell}$.

Similar analyses to the above for the non-perturbative annihilation cross-section, have been performed in refs. [65, 66], where also the Sommerfeld enhancement factors of eq. (4.27) have been computed for a Yukawa potential.

\section{Bound-state formation, de-excitation and decay rates for specific in- teractions}

We now focus on specific interactions and apply the formalism of the previous sections to calculate the BSF cross-sections, and the rates for de-excitation or decay of bound states, where relevant. We consider the interaction of two scalar particles (i) via a light scalar boson (section 5.1), and (ii) via an Abelian gauge vector boson (section 5.2). 
In the instantaneous approximation, these interactions are described in general by a Yukawa potential. $^{12}$ (Of course, an unbroken gauge symmetry gives rise to a Coulomb potential.) A Yukawa potential admits bound state solutions if $m_{\varphi}<\alpha \mu$, where $\alpha$ is the fine-structure constant of the interaction. On the other hand, the radiative formation of bound states via emission of a force mediator is kinematically possible if $m_{\varphi}<\left(\alpha^{2}+\right.$ $\left.v_{\text {rel }}^{2}\right) \mu / 2$; for $v_{\text {rel }}<\alpha$ - which is when the Sommerfeld effect renders bound-state formation efficient - this is a much stronger condition. Provided that this condition holds, the distortion of the wavefunctions due to the non-zero mediator mass, from their Coulomb limit, is expected to be negligible. For simplicity, we shall thus perform our computations in the Coulomb limit.

As is well known, in the presence of an attractive Coulomb potential

$$
V(\mathbf{r})=-\frac{\alpha}{r}, \quad \alpha>0,
$$

there is a discrete spectrum and a continuous spectrum of energy eigenstates. The continuous spectrum corresponds to the two-particle states, and is characterised by a continuous quantum number that stands for the expectation value of the momentum of the reduced system, $\mathbf{k}=\mu \mathbf{v}_{\text {rel }}$, with $\mathbf{v}_{\text {rel }}$ being the expectation value of the relative velocity. The discrete spectrum corresponds to the bound states, and is characterised by the integervalued quantum numbers $\{n \ell m\}$. In the discrete spectrum, the expectation value of the momentum of the reduced system is $\kappa / n$, with $\kappa \equiv \mu \alpha$ being the Bohr momentum. As we shall see, the parameter that essentially determines the efficiency of BSF is the ratio of the momentum expectation values of the bound and the scattering states,

$$
\zeta \equiv \frac{\kappa}{k}=\frac{\alpha}{v_{\text {rel }}} .
$$

The energies of the states of the discrete and the continuous spectra are

$$
\omega_{\mathbf{P}, n}=m+\frac{\mathbf{P}^{2}}{2 m}+\mathcal{E}_{n}, \quad \omega_{\mathbf{K}, \mathbf{k}}=m+\frac{\mathbf{K}^{2}}{2 m}+\mathcal{E}_{\mathbf{k}},
$$

where $\mathbf{P}, \mathbf{K}$ are the momenta of the CM of the bound and the two-particle states respectively, and

$$
\mathcal{E}_{n}=-\frac{\kappa^{2}}{2 \mu}=-\frac{\mu \alpha^{2}}{2 n^{2}}, \quad \mathcal{E}_{\mathbf{k}}=\frac{\mathbf{k}^{2}}{2 \mu}=\frac{\mu v_{\mathrm{rel}}^{2}}{2} .
$$

The wavefunctions are given in appendix F.1.

Useful integrals. For the calculation of the amplitudes $\mathcal{M}_{\mathbf{k} \rightarrow n}$ and $\mathcal{M}_{n^{\prime} \rightarrow n}$, we will find it useful to define the integrals

$$
\begin{aligned}
& \Xi_{1}(\mathbf{q}, \mathbf{p} ; K, P) \equiv \int \frac{d q^{0}}{2 \pi} S(q ; K) \int \frac{d p^{0}}{2 \pi} \tilde{S}_{1}\left(\eta_{1} P+p\right)(2 \pi) \delta\left(q^{0}-p^{0}-\eta_{2} P_{\varphi}^{0}\right) \\
& \Xi_{2}(\mathbf{q}, \mathbf{p} ; K, P) \equiv \int \frac{d q^{0}}{2 \pi} S(q ; K) \int \frac{d p^{0}}{2 \pi} \tilde{S}_{2}\left(\eta_{2} P-p\right)(2 \pi) \delta\left(q^{0}-p^{0}+\eta_{1} P_{\varphi}^{0}\right)
\end{aligned}
$$

\footnotetext{
${ }^{12}$ For a classification of the low-energy effective potentials generated by long-range interactions, and a systematic renormalisation procedure of singular potentials, see ref. [75].
} 
We evaluate $\Xi_{1}$ and $\Xi_{2}$ in appendix E. We will also need the following integrals involving the initial and final state wavefunctions

$$
\begin{aligned}
\mathcal{I}_{\mathbf{k}, n}(\mathbf{b}) & \equiv \int \frac{d^{3} p}{(2 \pi)^{3}} \tilde{\psi}_{n}^{\star}(\mathbf{p}) \tilde{\phi}_{\mathbf{k}}(\mathbf{p}+\mathbf{b}), \\
\mathcal{J}_{\mathbf{k}, n}(\mathbf{b}) & \equiv \int \frac{d^{3} p}{(2 \pi)^{3}} \mathbf{p} \tilde{\psi}_{n}^{\star}(\mathbf{p}) \tilde{\phi}_{\mathbf{k}}(\mathbf{p}+\mathbf{b}), \\
\mathcal{K}_{\mathbf{k}, n}(\mathbf{b}) & \equiv \int \frac{d^{3} p}{(2 \pi)^{3}} \mathbf{p}^{2} \tilde{\psi}_{n}^{\star}(\mathbf{p}) \tilde{\phi}_{\mathbf{k}}(\mathbf{p}+\mathbf{b}),
\end{aligned}
$$

and

$$
\begin{aligned}
\mathcal{I}_{n^{\prime}, n}(\mathbf{b}) & \equiv \int \frac{d^{3} p}{(2 \pi)^{3}} \tilde{\psi}_{n}^{\star}(\mathbf{p}) \tilde{\psi}_{n^{\prime}}(\mathbf{p}+\mathbf{b}), \\
\mathcal{J}_{n^{\prime}, n}(\mathbf{b}) & \equiv \int \frac{d^{3} p}{(2 \pi)^{3}} \mathbf{p} \tilde{\psi}_{n}^{\star}(\mathbf{p}) \tilde{\psi}_{n^{\prime}}(\mathbf{p}+\mathbf{b}) \\
\mathcal{K}_{n^{\prime}, n}(\mathbf{b}) & \equiv \int \frac{d^{3} p}{(2 \pi)^{3}} \mathbf{p}^{2} \tilde{\psi}_{n}^{\star}(\mathbf{p}) \tilde{\psi}_{n^{\prime}}(\mathbf{p}+\mathbf{b}) .
\end{aligned}
$$

We evaluate $\mathcal{I}, \mathcal{J}$ and $\mathcal{K}$ in appendix $\mathrm{F}$, for the initial and final states of interest. We shall use the integrals (5.5)-(5.12) in sections 5.1 and 5.2.

\subsection{Scalar mediator}

We consider the interaction Lagrangians

$$
\begin{aligned}
\delta \mathcal{L}_{S, r}= & \frac{1}{2} \partial_{\mu} \chi_{1} \partial^{\mu} \chi_{1}+\frac{1}{2} \partial_{\mu} \chi_{2} \partial^{\mu} \chi_{2}+\frac{1}{2} \partial_{\mu} \varphi \partial^{\mu} \varphi-\frac{1}{2} m_{1}^{2} \chi_{1}^{2}-\frac{1}{2} m_{2}^{2} \chi_{2}^{2}-\frac{1}{2} m_{\varphi}^{2} \phi^{2} \\
& -\frac{1}{2} g_{1} m_{1} \varphi \chi_{1}^{2}-\frac{1}{2} g_{2} m_{2} \varphi \chi_{2}^{2} \\
\delta \mathcal{L}_{S, c}= & \partial_{\mu} \chi_{1}^{\dagger} \partial^{\mu} \chi_{1}+\partial_{\mu} \chi_{2}^{\dagger} \partial^{\mu} \chi_{2}+\frac{1}{2} \partial_{\mu} \varphi \partial^{\mu} \varphi-m_{1}^{2}\left|\chi_{1}\right|^{2}-m_{2}^{2}\left|\chi_{2}\right|^{2}-\frac{1}{2} m_{\varphi}^{2} \phi^{2} \\
& -g_{1} m_{1} \varphi\left|\chi_{1}\right|^{2}-g_{2} m_{2} \varphi\left|\chi_{2}\right|^{2}
\end{aligned}
$$

In eq. (5.13), $\chi_{1}$ and $\chi_{2}$ are real scalar fields, while in eq. (5.14) they are complex. $\phi$ is a real scalar boson, with mass $m_{\varphi} \ll m_{1}, m_{2}$, and $g_{1}, g_{2}$ are dimensionless couplings. ${ }^{13}$

To lowest order, the interaction between $\chi_{1}$ and $\chi_{2}$ is mediated by one-boson exchange, as shown in figure 6 . Then

$$
\tilde{W}\left(p, p^{\prime} ; Q\right)=-\frac{i g_{1} g_{2} m_{1} m_{2}}{\left(p-p^{\prime}\right)^{2}-m_{\varphi}^{2}}
$$

\footnotetext{
${ }^{13}$ In eqs. (5.13), (5.14) and (5.48), we omit the quartic couplings in the scalar potential, since they do not enter our calculations.
} 


$$
\tilde{W}\left(p, p^{\prime} ; Q\right)=\overbrace{\eta_{2} Q-p}^{\eta_{1} Q+\underline{p}} \simeq \sum_{\eta_{2} Q-\frac{\sum_{\eta_{2}} Q}{\eta_{1} Q}-p^{\prime}}^{\eta_{1} Q+p^{\prime}}-p^{\prime}
$$

Figure 6. In the non-relativistic regime, the one-boson exchange is the dominant contribution to the $\chi_{1}-\chi_{2}$ interaction. Consequently, the 4-point function is approximated by an infinite ladder of one-boson exchange diagrams. The exchanged boson may be either scalar or vector.

In the instantaneous approximation ${ }^{14}$

$$
\mathcal{W}\left(\left|\mathbf{p}-\mathbf{p}^{\prime}\right|\right)=\frac{i g_{1} g_{2} m_{1} m_{2}}{\left(\mathbf{p}-\mathbf{p}^{\prime}\right)^{2}+m_{\varphi}^{2}}
$$

From eq. (2.86), we find the non-relativistic potential,

$$
V(\mathbf{r})=-\frac{1}{i 4 m \mu} \int \frac{d^{3} p}{(2 \pi)^{3}} \mathcal{W}(\mathbf{p}) e^{i \mathbf{p} \cdot \mathbf{r}}=-\frac{i g_{1} g_{2} m_{1} m_{2}}{i 4 m \mu} \int \frac{d^{3} p}{(2 \pi)^{3}} \frac{e^{i \mathbf{p} \cdot \mathbf{r}}}{\mathbf{p}^{2}+m_{\varphi}^{2}}
$$

That is,

$$
V(\mathbf{r})=-\frac{\alpha e^{-m_{\varphi} r}}{r}, \quad \text { with } \quad \alpha=\frac{g_{1} g_{2}}{16 \pi} .
$$

The interaction is attractive if $g_{1} g_{2}>0$, i.e. it is always attractive between particles of the same species or particles and antiparticles, but it can be either attractive or repulsive between particles of different species. As already mentioned, for our computations, we shall consider the limit $m_{\varphi} \rightarrow 0$.

\subsubsection{Bound-state formation amplitudes}

The lowest order contribution to the perturbative part of the radiative BSF amplitude arises from the diagrams shown in figure 7. In our approximation, the entire BSF amplitude corresponds to the ladder diagrams of figure 8. Recalling eqs. (3.16) and (3.17), the diagrams of figure 7 evaluate to

$$
\begin{aligned}
&(2 \pi)^{4} \delta^{4}\left(q_{1}+q_{2}-p_{1}-p_{2}-P_{\varphi}\right) i \mathcal{C}_{\varphi-\text { amp }}^{(5)}\left(P_{\varphi}, p_{1}, p_{2}, q_{1}, q_{2}\right) \\
&=-i g_{1} m_{1} \tilde{S}_{1}\left(q_{1}\right) \tilde{S}_{1}\left(p_{1}\right)(2 \pi)^{4} \delta^{4}\left(P_{\varphi}+p_{1}-q_{1}\right) \tilde{S}_{2}\left(q_{2}\right)(2 \pi)^{4} \delta^{4}\left(p_{2}-q_{2}\right) \\
& \quad-i g_{2} m_{2} \tilde{S}_{2}\left(p_{2}\right) \tilde{S}_{2}\left(q_{2}\right)(2 \pi)^{4} \delta^{4}\left(P_{\varphi}+p_{2}-q_{2}\right) \tilde{S}_{1}\left(q_{1}\right)(2 \pi)^{4} \delta^{4}\left(p_{1}-q_{1}\right) .
\end{aligned}
$$

\footnotetext{
${ }^{14}$ Note that the leading-order correction to the approximation of eq. (5.16), and similarly of eq. (5.50), are of order $\left(p^{0}-p^{\prime 0}\right)^{2}$. Due to the linearity of the Bethe-Salpeter eqs. (2.54), (2.55), the corresponding corrections to the approximations of the Bethe-Salpeter wavefunctions, $\tilde{\Psi}_{\mathbf{Q}, n}(p)$ and $\tilde{\Phi}_{\mathbf{Q}, \mathbf{q}}(p)$, that are introduced via eqs. (2.69) and (2.70), would also be of the same order. As discussed in earlier sections, in the regime of interest, $p^{0} \sim \mathcal{O}\left(\mathbf{p}^{2} / \mu\right)$, and such corrections would then be $\mathcal{O}\left(\mathbf{p}^{4} / \mu^{2}\right)$, i.e. of higher order than the corrections considered in eq. (3.33) and which appear in the S-matrix elements (3.30) and (3.31). This confirms the consistency of the approximation.
} 


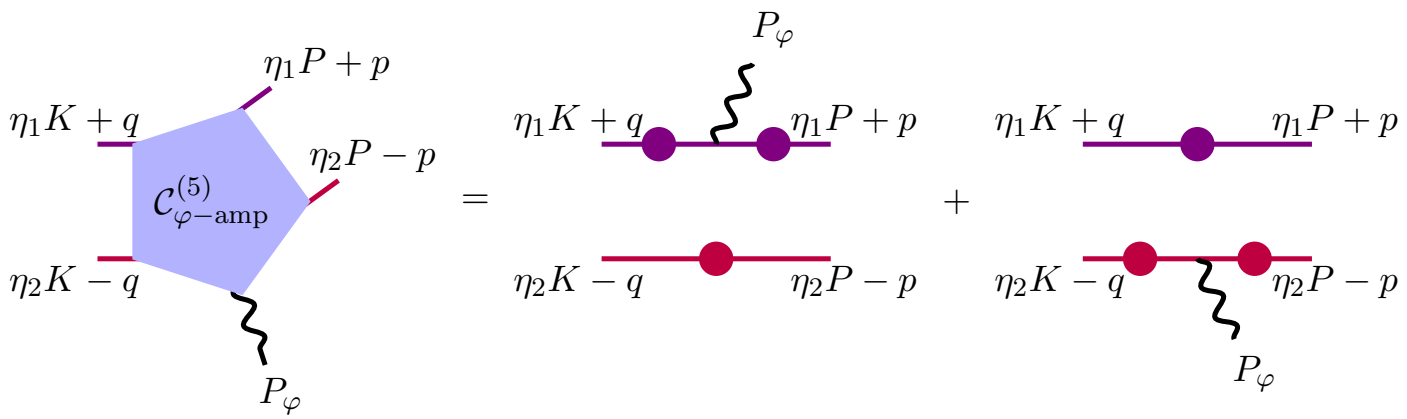

Figure 7. The lowest order contribution to level transition amplitudes, including bound-state formation.

Equivalently

$$
\begin{aligned}
\mathcal{C}_{\varphi-\mathrm{amp}}^{(5)}\left(P_{\varphi}, \eta_{1} P+p, \eta_{2} P-p, \eta_{1} K+q, \eta_{2} K-q\right) & \\
=-S(q ; K)\left[g _ { 1 } m _ { 1 } S _ { 1 } \left(\eta_{1} P\right.\right. & +p)(2 \pi)^{4} \delta^{4}\left(q-p-\eta_{2} P_{\varphi}\right) \\
& \left.\quad+g_{2} m_{2} S_{2}\left(\eta_{2} P-p\right)(2 \pi)^{4} \delta^{4}\left(q-p+\eta_{1} P_{\varphi}\right)\right] .
\end{aligned}
$$

Using the above, eq. (3.32) can be expressed in terms of the $\Xi_{1}, \Xi_{2}$ integrals defined in eqs. (5.5) and (5.6), as follows

$$
\begin{aligned}
& \mathcal{M}_{\text {trans }}(\mathbf{q} ; \mathbf{p})= \\
& \quad=-\frac{\left[g_{1} m_{1} \Xi_{1}(\mathbf{q}, \mathbf{p} ; K, P)(2 \pi)^{3} \delta^{3}\left(\mathbf{q}-\mathbf{p}-\eta_{2} \mathbf{P}_{\varphi}\right)+g_{2} m_{2} \Xi_{2}(\mathbf{q}, \mathbf{p} ; K, P)(2 \pi)^{3} \delta^{3}\left(\mathbf{q}-\mathbf{p}+\eta_{1} \mathbf{P}_{\varphi}\right)\right]}{\mathcal{S}_{0}(\mathbf{p} ; P) \mathcal{S}_{0}(\mathbf{q} ; K)} \\
& \quad \simeq-2 m \mu\left[1+\frac{\mathbf{p}^{2}}{2 \mu^{2}}\left(1-\frac{2 \mu}{m}\right)\right]\left[g_{1}(2 \pi)^{3} \delta^{3}\left(\mathbf{q}-\mathbf{p}-\eta_{2} \mathbf{P}_{\varphi}\right)+g_{2}(2 \pi)^{3} \delta^{3}\left(\mathbf{q}-\mathbf{p}+\eta_{1} \mathbf{P}_{\varphi}\right)\right],
\end{aligned}
$$

where in the second step, we used the non-relativistic approximations of $\Xi_{1}, \Xi_{2}$, given in eqs. (E.23), (E.24). Inserting this into eq. (3.30), we obtain

$$
\mathcal{M}_{\mathbf{k} \rightarrow n} \simeq-m \sqrt{2 \mu} \int \frac{d^{3} p}{(2 \pi)^{3}}\left(1+\frac{\mathbf{p}^{2}}{2 m \mu}\right) \tilde{\psi}_{n}^{\star}(\mathbf{p})\left[g_{1} \tilde{\phi}_{\mathbf{k}}\left(\mathbf{p}+\eta_{2} \mathbf{P}_{\varphi}\right)+g_{2} \tilde{\phi}_{\mathbf{k}}\left(\mathbf{p}-\eta_{1} \mathbf{P}_{\varphi}\right)\right] .
$$

The $\mathbf{p}^{2}$ term in the square brackets, will prove to be important in the cases of identical particles and particle-antiparticle pairs (see below). In terms of the integrals (5.7)-(5.9), eq. (5.20) becomes

$$
\mathcal{M}_{\mathbf{k} \rightarrow n} \simeq-m \sqrt{2 \mu}\left[g_{1} \mathcal{I}_{\mathbf{k}, n}\left(\eta_{2} \mathbf{P}_{\varphi}\right)+g_{2} \mathcal{I}_{\mathbf{k}, n}\left(-\eta_{1} \mathbf{P}_{\varphi}\right)+\frac{g_{1} \mathcal{K}_{\mathbf{k}, n}\left(\eta_{2} \mathbf{P}_{\varphi}\right)+g_{2} \mathcal{K}_{\mathbf{k}, n}\left(-\eta_{1} \mathbf{P}_{\varphi}\right)}{2 m \mu}\right] .
$$

Capture in the ground state. For capture in the $\{100\}$ state, eq. (5.21) becomes

$$
\begin{aligned}
& \mathcal{M}_{\mathbf{k} \rightarrow\{100\}} \simeq-m \sqrt{2 \mu}\left\{\left[g_{1} \mathcal{I}_{\mathbf{k},\{100\}}\left(\eta_{2} \mathbf{P}_{\varphi}\right)+g_{2} \mathcal{I}_{\mathbf{k},\{100\}}\left(-\eta_{1} \mathbf{P}_{\varphi}\right)\right]\left(1-\frac{\mu \alpha^{2}}{2 m}\right)\right. \\
&\left.+\frac{g_{1} \overline{\mathcal{K}}_{\mathbf{k}}\left(\eta_{2} \mathbf{P}_{\varphi}\right)+g_{2} \overline{\mathcal{K}}_{\mathbf{k}}\left(-\eta_{1} \mathbf{P}_{\varphi}\right)}{2 m \mu}\right\},
\end{aligned}
$$


where we used eq. (F.22). We shall drop the $\alpha^{2}$ correction in the coefficient of the $\mathcal{I}_{\mathbf{k},\{100\}}$ integrals. The mediator momentum is $\left|\mathbf{P}_{\varphi}\right|=\mathcal{E}_{\mathbf{k}}-\mathcal{E}_{\{100\}}=\left(1+\zeta^{2}\right) k^{2} /(2 \mu)$ (cf. eqs. (3.41), (5.4)). Using eqs. (F.40) and (F.42) for the integrals $\mathcal{I}_{\mathbf{k},\{100\}}$ and $\overline{\mathcal{K}}_{\mathbf{k}}$, we find

$$
\begin{aligned}
\mathcal{M}_{\mathbf{k} \rightarrow\{100\}} \simeq & -\mathcal{R}(\zeta) \sqrt{\frac{2 m^{2}}{\mu k}} \times\left[\left(g_{1} \eta_{2}-g_{2} \eta_{1}\right) \cos \theta\right. \\
& \left.+\frac{k}{2 \mu}\left\{\left(g_{1} \eta_{2}^{2}+g_{2} \eta_{1}^{2}\right)\left[(-1+i \zeta)+2(2-i \zeta) \cos ^{2} \theta\right]+\frac{\mu}{m}\left(g_{1}+g_{2}\right)(1+i \zeta)\right\}\right],
\end{aligned}
$$

where $\theta$ is the angle between $\mathbf{k}$ and $\mathbf{P}_{\varphi}$, and $\mathcal{R}(\zeta)$ is given in eq. (F.36). (We emphasise that the above expression is not a consistent expansion in $\alpha$, but rather only in $v_{\text {rel }}^{2}$ ). We discern the following cases:

- For a particle-antiparticle pair, or for identical particles, $g_{1}=g_{2}=g, \eta_{1}=\eta_{2}=1 / 2$ and $\mu=m / 4$; the first term in eq. (5.22) vanishes, and we obtain

$$
\begin{aligned}
\mathcal{M}_{\mathbf{k} \rightarrow\{100\}} & \simeq-8 \sqrt{2 \pi \zeta} \mathcal{R}(\zeta) v_{\text {rel }}\left[i \zeta+(2-i \zeta) \cos ^{2} \theta\right] \\
& =-\frac{16 \sqrt{2 \pi \zeta} \mathcal{R}(\zeta)}{3} v_{\text {rel }}\left[(1+i \zeta) P_{0}(\cos \theta)+(2-i \zeta) P_{2}(\cos \theta)\right],
\end{aligned}
$$

where in the second line, we decomposed the amplitude in partial waves.

- For non-degenerate particles, the first term in eq. (5.22) dominates, and

$$
\mathcal{M}_{\mathbf{k} \rightarrow\{100\}} \simeq-4 \sqrt{2 \pi \zeta} \mathcal{R}(\zeta)\left(\frac{m}{\mu}\right)\left[\frac{\left(g_{1} \eta_{2}-g_{2} \eta_{1}\right)^{2}}{16 \pi \alpha}\right]^{1 / 2} P_{1}(\cos \theta)
$$

(The factor inside the square brackets becomes equal to 1 in the limit $g_{1}=g_{2}$, $\eta_{1} \gg \eta_{2}$.)

Capture in excited state with non-zero angular momentum. Since for a pair of degenerate particles, the cross-section for radiative capture to the ground state is either $v_{\text {rel }}^{2}$ or $\alpha^{2}$ suppressed (as seen by comparing eqs. (5.23) and (5.24)), we shall now calculate the amplitude for capture in the $\{210\}$ state. In this case, $\left|\mathbf{P}_{\varphi}\right|=\mathcal{E}_{\mathbf{k}}-\mathcal{E}_{\{210\}}=\left(4+\zeta^{2}\right) k^{2} /(8 \mu)$. From eq. (5.21), and keeping only the leading order terms of eq. (F.43) for the $\mathcal{I}_{\mathbf{k},\{210\}}$ integrals, we find

$$
\begin{aligned}
\mathcal{M}_{\mathbf{k} \rightarrow\{210\}} \simeq & -m \sqrt{2 \mu}\left[g_{1} \mathcal{I}_{\mathbf{k},\{210\}}\left(\eta_{2} \mathbf{P}_{\varphi}\right)+g_{2} \mathcal{I}_{\mathbf{k},\{210\}}\left(-\eta_{1} \mathbf{P}_{\varphi}\right)\right] \\
\simeq & \frac{m}{\mu}\left(\frac{g_{1} \eta_{2}+g_{2} \eta_{1}}{\sqrt{16 \pi \alpha}}\right) \frac{2^{7} \pi i}{3} \frac{\zeta^{4}(2-i \zeta) e^{\pi \zeta / 2} \Gamma(1-i \zeta)}{\left(4+\zeta^{2}\right)^{3}}\left(\frac{i \zeta+2}{i \zeta-2}\right)^{-i \zeta} \\
& \times\left[(i \zeta+2) P_{0}(\cos \theta)+8(i \zeta-1) P_{2}(\cos \theta)\right] .
\end{aligned}
$$

The $J=0$ and $J=2$ contributions to the above yield the squared amplitudes

$$
\begin{aligned}
\left|\mathcal{M}_{\mathbf{k} \rightarrow\{210\}, J=0}\right|^{2} & \simeq \frac{m^{2}}{\mu^{2}}\left[\frac{\left(g_{1} \eta_{2}+g_{2} \eta_{1}\right)^{2}}{16 \pi \alpha}\right] \frac{2^{19} \pi^{5}}{3^{2}} \frac{\zeta^{9}}{\left(4+\zeta^{2}\right)^{4}} \frac{e^{-4 \zeta \operatorname{arccot}(\zeta / 2)}}{1-e^{-2 \pi \zeta}} \\
\left|\mathcal{M}_{\mathbf{k} \rightarrow\{210\}, J=2}\right|^{2} & \simeq \frac{m^{2}}{\mu^{2}}\left[\frac{\left(g_{1} \eta_{2}+g_{2} \eta_{1}\right)^{2}}{16 \pi \alpha}\right] \frac{2^{25} \pi^{5}}{3^{2} 5^{2}} \frac{\zeta^{9}\left(1+\zeta^{2}\right)}{\left(4+\zeta^{2}\right)^{5}} \frac{e^{-4 \zeta \operatorname{arccot}(\zeta / 2)}}{1-e^{-2 \pi \zeta}}
\end{aligned}
$$




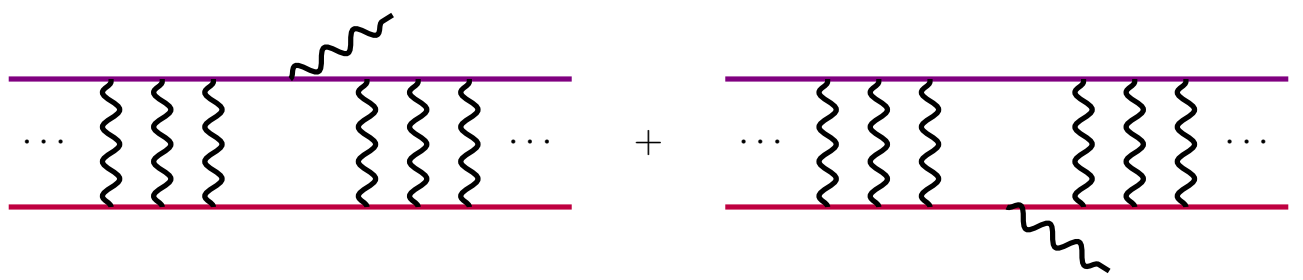

Figure 8. The ladder diagrams giving the dominant contribution to bound-state formation and other level transitions. The mediator can be either a scalar or a vector boson.

(We remind that $\mathcal{M}_{J}$ is defined in eq. (3.45), and note that the factor inside the square brackets becomes equal to 1 in the limit $g_{1}=g_{2}$, independently of $\eta_{1}, \eta_{2}$.)

\subsubsection{Bound-state formation cross-sections and partial-wave unitarity}

Combining eq. (3.47) for the partial-wave cross-section and the amplitudes (5.23)-(5.26), we obtain

- For $g_{1}=g_{2}=g$ and $\eta_{1}=\eta_{2}=1 / 2$ (i.e. $\mu=m / 4$ ),

$$
\begin{aligned}
\sigma_{\mathrm{BSF}, J=0}^{\{100\}} & \simeq \frac{2^{7} \pi^{2} \alpha^{3}}{3^{2} \mu^{2}} \frac{\zeta^{4}}{1+\zeta^{2}} \frac{e^{-4 \zeta \operatorname{arccot} \zeta}}{1-e^{-2 \pi \zeta}}, \\
\sigma_{\mathrm{BSF}, J=2}^{\{100\}} & \simeq \frac{2^{7} \pi^{2} \alpha^{3}}{3^{2} 5 \mu^{2}} \frac{\zeta^{4}\left(4+\zeta^{2}\right)}{\left(1+\zeta^{2}\right)^{2}} \frac{e^{-4 \zeta \operatorname{arccot} \zeta}}{1-e^{-2 \pi \zeta}}, \\
\sigma_{\mathrm{BSF}}^{\{100\}} \simeq \sigma_{\mathrm{BSF}, J=0}^{\{100\}}+\sigma_{\mathrm{BSF}, J=2}^{\{100\}} & \simeq \frac{2^{7} \pi^{2} \alpha^{3}}{15 \mu^{2}} \frac{\zeta^{4}\left(3+2 \zeta^{2}\right)}{\left(1+\zeta^{2}\right)^{2}} \frac{e^{-4 \zeta \operatorname{arccot} \zeta}}{1-e^{-2 \pi \zeta}} .
\end{aligned}
$$

- For non-degenerate particles,

$$
\sigma_{\mathrm{BSF}}^{\{100\}} \simeq \sigma_{\mathrm{BSF}, J=1}^{\{100\}} \simeq \frac{2^{7} \pi^{2} \alpha}{3 \mu^{2}}\left[\frac{\left(g_{1} \eta_{2}-g_{2} \eta_{1}\right)^{2}}{16 \pi \alpha}\right] \frac{\zeta^{6}}{\left(1+\zeta^{2}\right)^{2}} \frac{e^{-4 \zeta \operatorname{arccot} \zeta}}{1-e^{-2 \pi \zeta}}
$$

- For capture to the $\{210\}$ state,

$$
\begin{aligned}
\sigma_{\mathrm{BSF}, J=0}^{\{210\}} & \simeq \frac{2^{8} \pi^{2} \alpha}{3^{2} \mu^{2}}\left[\frac{\left(g_{1} \eta_{2}+g_{2} \eta_{1}\right)^{2}}{16 \pi \alpha}\right] \frac{\zeta^{8}}{\left(4+\zeta^{2}\right)^{3}} \frac{e^{-4 \zeta \operatorname{arccot}(\zeta / 2)}}{1-e^{-2 \pi \zeta}}, \\
\sigma_{\mathrm{BSF}, J=2}^{\{210\}} & \simeq \frac{2^{14} \pi^{2} \alpha}{3^{2} 5 \mu^{2}}\left[\frac{\left(g_{1} \eta_{2}+g_{2} \eta_{1}\right)^{2}}{16 \pi \alpha}\right] \frac{\zeta^{8}\left(1+\zeta^{2}\right)}{\left(4+\zeta^{2}\right)^{4}} \frac{e^{-4 \zeta \operatorname{arccot}(\zeta / 2)}}{1-e^{-2 \pi \zeta}}, \\
\sigma_{\mathrm{BSF}}^{\{210\}} & \simeq \sigma_{\mathrm{BSF}, J=0}^{\{210\}}+\sigma_{\mathrm{BSF}, J=2}^{\{210\}} \\
& \simeq \frac{2^{8} \pi^{2} \alpha}{15 \mu^{2}}\left[\frac{\left(g_{1} \eta_{2}+g_{2} \eta_{1}\right)^{2}}{16 \pi \alpha}\right] \frac{\zeta^{8}\left(28+23 \zeta^{2}\right)}{\left(4+\zeta^{2}\right)^{4}} \frac{e^{-4 \zeta \operatorname{arccot}(\zeta / 2)}}{1-e^{-2 \pi \zeta}} .
\end{aligned}
$$


Unitarity limit. For $\zeta \gg 1$, eqs. (5.27), (5.28), (5.30), (5.31) and (5.32) become

$$
\begin{aligned}
& \left.\sigma_{\mathrm{BSF}, J=0}^{\{100\}} \rightarrow \frac{\pi}{\mu^{2} v_{\mathrm{rel}}^{2}} \times \frac{2^{7} \pi \alpha^{5}}{3^{2} e^{4}} \quad \text { (degenerate particles: } g_{1}=g_{2}, \eta_{1}=\eta_{2}=1 / 2\right), \\
& \sigma_{\mathrm{BSF}, J=2}^{\{100\}} \rightarrow \frac{5 \pi}{\mu^{2} v_{\mathrm{rel}}^{2}} \times \frac{2^{7} \pi \alpha^{5}}{3^{2} 5^{2} e^{4}} \quad\left(\text { degenerate particles: } g_{1}=g_{2}, \eta_{1}=\eta_{2}=1 / 2\right), \\
& \sigma_{\mathrm{BSF}, J=1}^{\{100\}} \rightarrow \frac{3 \pi}{\mu^{2} v_{\mathrm{rel}}^{2}} \times \frac{2^{7} \pi \alpha^{3}}{3^{2} e^{4}}\left[\frac{\left(g_{1} \eta_{2}-g_{2} \eta_{1}\right)^{2}}{16 \pi \alpha}\right] \quad \text { (non-degenerate particles), } \\
& \sigma_{\mathrm{BSF}, J=0}^{\{210\}} \rightarrow \frac{\pi}{\mu^{2} v_{\mathrm{rel}}^{2}} \times \frac{2^{8} \pi \alpha^{3}}{3^{2} e^{8}}\left[\frac{\left(g_{1} \eta_{2}+g_{2} \eta_{1}\right)^{2}}{16 \pi \alpha}\right], \\
& \sigma_{\mathrm{BSF}, J=2}^{\{210\}} \rightarrow \frac{5 \pi}{\mu^{2} v_{\mathrm{rel}}^{2}} \times \frac{2^{14} \pi \alpha^{3}}{3^{2} 5^{2} e^{8}}\left[\frac{\left(g_{1} \eta_{2}+g_{2} \eta_{1}\right)^{2}}{16 \pi \alpha}\right] .
\end{aligned}
$$

It is interesting to note that in this low-velocity regime, the velocity dependence of all partial waves is the same. ${ }^{15}$ This is in fact expected by unitarity, since $\zeta \gg 1$ is both the large coupling and the low-velocity limit. Indeed, the unitarity bounds on the partial-wave inelastic cross-sections, shown in eq. (3.48), all have the same velocity dependence. They are realised when the factors to the right of the $\times$ symbols in eqs. (5.34)-(5.38) become $\approx 1$. The validity of our calculation is thus limited to at most $\alpha \lesssim \alpha_{\text {uni }}$, with the strongest bound, $\alpha_{\text {uni }} \approx 1$, obtained from $\sigma_{\mathrm{BSF}, J=0}^{\{100\}}$.

\subsubsection{De-excitation rate}

The radiative capture to the $\{210\}$ state is the dominant BSF process for particleantiparticle pairs and pairs of self-conjugate particles. Moreover, for non-degenerate particles, it is slower but comparable to the capture to the ground state. Here, we shall thus compute the de-excitation rate of the $\{210\}$ state.

The radiative de-excitation of a bound state arises from the same diagrams as the radiative capture to a bound state, albeit for different initial and final states. In our approximation, these are the ladder diagrams shown in figure 8. Inserting eq. (5.19) into eq. (3.31), we find

$$
\mathcal{M}_{n^{\prime} \rightarrow n} \simeq-m\left\{g_{1} \mathcal{I}_{n^{\prime}, n}\left(\eta_{2} \mathbf{P}_{\varphi}\right)+g_{2} \mathcal{I}_{n^{\prime}, n}\left(-\eta_{1} \mathbf{P}_{\varphi}\right)+\frac{g_{1} \mathcal{K}_{n^{\prime}, n}\left(\eta_{2} \mathbf{P}_{\varphi}\right)+g_{2} \mathcal{K}_{n^{\prime}, n}\left(-\eta_{1} \mathbf{P}_{\varphi}\right)}{2 m \mu}\right\} .
$$

In the $\{210\} \rightarrow\{100\}$ transition, the mediator is emitted with momentum $\left|\mathbf{P}_{\varphi}\right|=$ $\mathcal{E}_{\{210\}}-\mathcal{E}_{\{100\}}=(3 / 8) \mu \alpha^{2}$. Then, using eq. (F.46), we find

$$
\begin{aligned}
\mathcal{M}_{\{210\} \rightarrow\{100\}} & \simeq-m\left[g_{1} \mathcal{I}_{\{210\},\{100\}}\left(\eta_{2} \mathbf{P}_{\varphi}\right)+g_{2} \mathcal{I}_{\{210\},\{100\}}\left(-\eta_{1} \mathbf{P}_{\varphi}\right)\right] \\
& \simeq \frac{i 2^{6} m \alpha \sqrt{2 \pi \alpha}}{3^{4}}\left[\frac{g_{1} \eta_{2}+g_{2} \eta_{1}}{\sqrt{16 \pi \alpha}}\right] .
\end{aligned}
$$

The de-excitation rate, given by eq. (3.49), becomes

$$
\Gamma_{\{210\} \rightarrow\{100\}+\varphi} \simeq \frac{2^{7} \alpha^{5} \mu}{3^{7}}\left[\frac{\left(g_{1} \eta_{2}+g_{2} \eta_{1}\right)^{2}}{16 \pi \alpha}\right] .
$$

\footnotetext{
${ }^{15}$ This was also noted in ref. [66], for Sommerfeld-enhanced annihilation processes.
} 


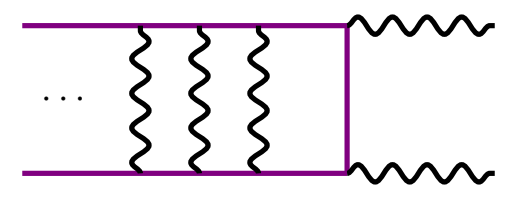

Figure 9. Annihilation of an unbound particle-antiparticle pair or decay of a bound particleantiparticle pair into two force mediators. The mediator may be either a scalar or a vector boson.

\subsubsection{Annihilation vs bound-state formation for particle-antiparticle pairs}

A particle-antiparticle pair or a pair of self-conjugate identical particles coupled to a light scalar, can annihilate into two mediators, $\chi^{\dagger} \chi \rightarrow \varphi \varphi$ or $\chi \chi \rightarrow \varphi \varphi$, as shown in figure 9 . In both cases, the perturbative annihilation amplitude, evaluated in the CM frame, in the non-relativistic regime, is

$$
\hat{\mathcal{M}}_{\mathrm{ann}}^{\mathrm{pert}}(\mathbf{q},-\mathbf{q} ; \mathbf{p},-\mathbf{p}) \simeq a_{0}+\frac{a_{1}}{m \mu}(\mathbf{q} \cdot \mathbf{p})
$$

with $a_{0} \simeq a_{1} \simeq 16 \pi \alpha$. Using eqs. (4.24), (4.26) and (4.27), we find that the annihilation cross-section is $s$-wave dominated, $\sigma_{\text {ann }} v_{\text {rel }} \simeq \sigma_{0} S_{0, \text { ann }}$, with $\sigma_{0}=f_{s}\left|a_{0}\right|^{2} /(32 \pi m \mu) \simeq$ $\pi \alpha^{2} / \mu^{2}$ being the perturbative $s$-wave annihilation cross-section, and $S_{0, \text { ann }}=\left|\phi_{\mathbf{k}}(0)\right|^{2}$ being the $s$-wave Sommerfeld-enhancement factor for annihilation. Using the wavefunction of eq. (F.11), we obtain

$$
\sigma_{\mathrm{ann}} v_{\mathrm{rel}} \simeq \frac{\pi \alpha^{2}}{\mu^{2}} \frac{2 \pi \zeta}{1-e^{-2 \pi \zeta}}
$$

Note that here, $\mu=m_{\chi} / 2=m / 4$, with $m_{\chi}$ being the mass of the annihilating particles.

We may now compare the BSF and annihilation cross-sections,

$$
\begin{aligned}
& \frac{\sigma_{\mathrm{BSF}}^{\{100\}}}{\sigma_{\mathrm{ann}}} \simeq \frac{2^{6} \alpha^{2} \zeta^{2}\left(3+2 \zeta^{2}\right)}{15\left(1+\zeta^{2}\right)^{2}} e^{-4 \zeta \operatorname{arccot} \zeta}, \\
& \frac{\sigma_{\mathrm{BSF}}^{\{210\}}}{\sigma_{\mathrm{ann}}} \simeq \frac{2^{7} \zeta^{6}\left(28+23 \zeta^{2}\right)}{15\left(4+\zeta^{2}\right)^{4}} e^{-4 \zeta \operatorname{arccot}(\zeta / 2)} .
\end{aligned}
$$

At $\zeta \gg 1, \sigma_{\mathrm{BSF}}^{\{100\}} / \sigma_{\mathrm{ann}} \simeq 0.15 \alpha^{2}$ and $\sigma_{\mathrm{BSF}}^{\{210\}} / \sigma_{\mathrm{ann}} \simeq 0.066 .{ }^{16}$ We compare $\sigma_{\mathrm{ann}}$ and $\sigma_{\mathrm{BSF}}^{\{210\}}$ in figure 1. Since annihilation is the dominant inelastic process, it lowers the value of $\alpha$ at which the unitarity bound appears to be realised, to $\alpha_{\text {uni }} \approx 0.54$.

\subsubsection{Particle-antiparticle bound-state decay rates}

From (4.25), (4.26) and (4.28), we find that the decay of the $\{100\}$ particle-antiparticle bound state into two mediators, is dominated by the $s$-wave contribution,

$$
\Gamma_{\{100\} \rightarrow \varphi \varphi}=\sigma_{0}\left|\psi_{100}(0)\right|^{2}=\frac{\pi \alpha^{2}}{\mu^{2}} \frac{\kappa^{3}}{\pi}=\mu \alpha^{5} .
$$

\footnotetext{
${ }^{16}$ Note that for a Dirac fermion-antifermion pair, the annihilation into scalars is dominantly $p$-wave. The spin-averaged annihilation cross-section times relative velocity is $\sigma_{\text {ann }} v_{\text {rel }} \simeq \sigma_{1} S_{1 \text {, ann }}$, with $\sigma_{1} \simeq 3 \pi \alpha^{2} /\left(2 \mu^{2}\right)$ and $S_{1, \text { ann }}=v_{\text {rel }}^{2}\left(1+\zeta^{2}\right) 2 \pi \zeta /\left(1-e^{-2 \pi \zeta}\right)$. On the other hand, the BSF cross-sections do not depend on the spins of the interacting particles, since in the non-relativistic regime, the spin is conserved separately from the orbital angular momentum. Thus, for Dirac fermions, at $\zeta \gg 1, \sigma_{\mathrm{BSF}}^{\{100\}} / \sigma_{\mathrm{ann}} \simeq 0.1$ and $\sigma_{\mathrm{BSF}}^{\{210\}} / \sigma_{\mathrm{ann}} \simeq$ $0.044 / \alpha^{2}$. This means that for $\alpha \lesssim 0.2$, BSF is faster than annihilation in the regime where the Sommerfeld effect is important.
} 
For the $\{210\}$ bound state, the $s$-wave decay mode vanishes, since $\psi_{210}(0)=0$. However, a non-vanishing contribution arises from the $p$-wave mode. From eq. (4.26), we find $\sigma_{1}=f_{s}\left|a_{1}\right|^{2} /\left(2^{7} 3 \pi m \mu\right)=\pi \alpha^{2} /\left(12 \mu^{2}\right)$. From eq. (4.28) and the wavefunction (F.9), we obtain $S_{1, \mathrm{dec}}=\kappa^{5} /\left(32 \pi \mu^{2}\right)$. Then

$$
\Gamma_{\{210\} \rightarrow \varphi \varphi}=\sigma_{1} S_{1, \mathrm{dec}}=\frac{\pi \alpha^{2}}{12 \mu^{2}} \frac{\kappa^{5}}{32 \pi \mu^{2}}=\frac{\mu \alpha^{7}}{2^{7} 3} .
$$

The decay rates into three mediators are expected to be suppressed by one additional power of $\alpha$ with respect to the above. Recalling eq. (5.41), this suggests that for the excited state $\{210\}$, the transition to the ground state is the dominant decay mode.

\subsection{Vector mediator}

We now consider two scalar particles $\chi_{1}, \chi_{2}$ coupled to a gauged $\mathrm{U}(1)$ force. The interaction Lagrangian is

$$
\delta \mathcal{L}_{V}=\left(D_{\mu} \chi_{1}\right)^{\dagger}\left(D^{\mu} \chi_{1}\right)+\left(D_{\mu} \chi_{2}\right)^{\dagger}\left(D^{\mu} \chi_{2}\right)-\frac{1}{4} F_{\mu \nu} F^{\mu \nu}-m_{1}^{2}\left|\chi_{1}\right|^{2}-m_{2}^{2}\left|\chi_{2}\right|^{2}
$$

where $\chi_{1}, \chi_{2}$ are complex scalar bosons, $F^{\mu \nu}=\partial^{\mu} \varphi^{\nu}-\partial^{\nu} \varphi^{\mu}$ and $D^{\mu}=\partial^{\mu}-i c_{j} g \varphi^{\mu}$, with $c_{1}, c_{2}$ being the charges of $\chi_{1}, \chi_{2} \cdot{ }^{13}$

The one-boson exchange diagram gives

$$
\tilde{W}\left(p, p^{\prime} ; Q\right)=\frac{i c_{1} c_{2} g^{2}}{\left(p-p^{\prime}\right)^{2}}\left[\left(2 \eta_{1} Q+p+p^{\prime}\right) \cdot\left(2 \eta_{2} Q-p-p^{\prime}\right)\right]
$$

In the non-relativistic regime, we shall approximate the above with

$$
\tilde{W}\left(p, p^{\prime} ; Q\right) \simeq \frac{i 4 \eta_{1} \eta_{2} Q^{2} c_{1} c_{2} g^{2}}{\left(p-p^{\prime}\right)^{2}}
$$

In the instantaneous approximation, and setting $Q^{2} \simeq m^{2}$

$$
\mathcal{W}\left(\left|\mathbf{p}-\mathbf{p}^{\prime}\right|\right) \simeq-\frac{i 4 \eta_{1} \eta_{2} c_{1} c_{2} g^{2} m^{2}}{\left(\mathbf{p}-\mathbf{p}^{\prime}\right)^{2}}
$$

and

$$
V(\mathbf{r})=-\frac{1}{i 4 m \mu} \int \frac{d^{3} p}{(2 \pi)^{3}} \mathcal{W}(\mathbf{p}) e^{i \mathbf{p} \cdot \mathbf{r}}=c_{1} c_{2} g^{2} \int \frac{d^{3} p}{(2 \pi)^{3}} \frac{e^{i \mathbf{p} \cdot \mathbf{r}}}{\mathbf{p}^{2}}
$$

That is,

$$
V(\mathbf{r})=-\frac{\alpha}{r}, \quad \text { with } \quad \alpha=-\frac{c_{1} c_{2} g^{2}}{4 \pi} .
$$

The interaction is attractive if $c_{1} c_{2}<0$. 


\subsubsection{Bound-state formation amplitude, cross-section and partial-wave uni- tarity}

The perturbative part of the level-transition amplitudes is $\mathcal{C}_{\varphi \text {-amp }}^{(5)}=\epsilon_{\mu} \mathcal{C}_{\varphi \text {-amp }}^{\mu}$, with the lowest order contribution depicted in figure 7,

$$
\begin{aligned}
& (2 \pi)^{4} \delta^{4}\left(q_{1}+q_{2}-p_{1}-p_{2}-P_{\varphi}\right) i \mathcal{C}_{\varphi-\mathrm{amp}}^{\mu}\left(P_{\varphi}, p_{1}, p_{2}, q_{1}, q_{2}\right) \simeq \\
& \simeq-i g c_{1}\left(p_{1}^{\mu}+q_{1}^{\mu}\right) \tilde{S}_{1}\left(p_{1}\right) \tilde{S}_{1}\left(q_{1}\right)(2 \pi)^{4} \delta^{4}\left(P_{\varphi}+p_{1}-q_{1}\right) \tilde{S}_{2}\left(q_{2}\right)(2 \pi)^{4} \delta^{4}\left(p_{2}-q_{2}\right) \\
& \quad-i g c_{2}\left(p_{2}^{\mu}+q_{2}^{\mu}\right) \tilde{S}_{2}\left(p_{2}\right) \tilde{S}_{2}\left(q_{2}\right)(2 \pi)^{4} \delta^{4}\left(P_{\varphi}+p_{2}-q_{2}\right) \tilde{S}_{1}\left(q_{1}\right)(2 \pi)^{4} \delta^{4}\left(p_{1}-q_{1}\right) .
\end{aligned}
$$

From this, we obtain

$$
\begin{aligned}
\mathcal{C}_{\varphi-\text { amp }}^{\mu}\left(P_{\varphi}, \eta_{1} P+p, \eta_{2} P-p, \eta_{1} K+q, \eta_{2} K-q\right)= \\
=-g S(q ; K)\left\{c_{1}\left[2 \eta_{1} K^{\mu}-\left(\eta_{1}-\eta_{2}\right) P_{\varphi}^{\mu}+2 p^{\mu}\right] \tilde{S}_{1}\left(\eta_{1} P+p\right)(2 \pi)^{4} \delta^{4}\left(q-p-\eta_{2} P_{\varphi}\right)\right. \\
\left.+c_{2}\left[2 \eta_{2} K^{\mu}+\left(\eta_{1}-\eta_{2}\right) P_{\varphi}^{\mu}-2 p^{\mu}\right] \tilde{S}_{2}\left(\eta_{2} P-p\right)(2 \pi)^{4} \delta^{4}\left(q-p+\eta_{1} P_{\varphi}\right)\right\} .
\end{aligned}
$$

Then, $\mathcal{M}_{\text {trans }}=\epsilon_{\mu} \mathcal{M}_{\text {trans }}^{\mu}$, where for $j=1,2,3$,

$$
\begin{aligned}
\mathcal{M}_{\text {trans }}^{j}(\mathbf{q} ; \mathbf{p})=- & \frac{g}{\mathcal{S}_{0}(\mathbf{q} ; K) \mathcal{S}_{0}(\mathbf{p} ; P)} \\
& \times\left\{c_{1}\left[2 \eta_{1} K^{j}-\left(\eta_{1}-\eta_{2}\right) P_{\varphi}^{j}+2 p^{j}\right] \Xi_{1}(\mathbf{q}, \mathbf{p} ; K, P)(2 \pi)^{3} \delta^{3}\left(\mathbf{q}-\mathbf{p}-\eta_{2} \mathbf{P}_{\varphi}\right)\right. \\
& \left.+c_{2}\left[2 \eta_{2} K^{j}+\left(\eta_{1}-\eta_{2}\right) P_{\varphi}^{j}-2 p^{j}\right] \Xi_{2}(\mathbf{q}, \mathbf{p} ; K, P)(2 \pi)^{3} \delta^{3}\left(\mathbf{q}-\mathbf{p}+\eta_{1} \mathbf{P}_{\varphi}\right)\right\}
\end{aligned}
$$

We remind that $\Xi_{1}, \Xi_{2}$ are defined in eqs. (5.5), (5.6). Using their non-relativistic approximations (E.23), (E.24), we find the amplitude of eq. (3.30) to be $\mathcal{M}_{\mathbf{k} \rightarrow n}=\epsilon_{\mu} \mathcal{M}_{\mathbf{k} \rightarrow n}^{\mu}$, where for $j=1,2,3$,

$$
\begin{aligned}
\mathcal{M}_{\mathbf{k} \rightarrow n}^{j}=-g \sqrt{2 \mu} \int \frac{d^{3} p}{(2 \pi)^{3}} \tilde{\psi}_{n}^{\star}(\mathbf{p})\{ & \frac{c_{1}}{\eta_{1}}\left[2 \eta_{1} K^{j}-\left(\eta_{1}-\eta_{2}\right) P_{\varphi}^{j}+2 p^{j}\right] \tilde{\phi}_{\mathbf{k}}\left(\mathbf{p}+\eta_{2} \mathbf{P}_{\varphi}\right) \\
& \left.+\frac{c_{2}}{\eta_{2}}\left[2 \eta_{2} K^{j}+\left(\eta_{1}-\eta_{2}\right) P_{\varphi}^{j}-2 p^{j}\right] \tilde{\phi}_{\mathbf{k}}\left(\mathbf{p}-\eta_{1} \mathbf{P}_{\varphi}\right)\right\} .
\end{aligned}
$$

We may rewrite the above in terms of the $\mathcal{I}, \mathcal{J}$ integrals, defined in eqs. (5.7) and (5.8), as follows

$$
\begin{aligned}
\mathcal{M}_{\mathbf{k} \rightarrow n}^{j}= & -2 g \sqrt{2 \mu}\left\{\frac{c_{1}}{\eta_{1}} \mathcal{J}_{\mathbf{k}, n}^{j}\left(\eta_{2} \mathbf{P}_{\varphi}\right)-\frac{c_{2}}{\eta_{2}} \mathcal{J}_{\mathbf{k}, n}^{j}\left(-\eta_{1} \mathbf{P}_{\varphi}\right)\right. \\
& \left.+\left[c_{1}\left(K^{j}-\frac{\eta_{1}-\eta_{2}}{2 \eta_{1}} P_{\varphi}^{j}\right) \mathcal{I}_{\mathbf{k}, n}\left(\eta_{2} \mathbf{P}_{\varphi}\right)+c_{2}\left(K^{j}+\frac{\eta_{1}-\eta_{2}}{2 \eta_{2}} P_{\varphi}^{j}\right) \mathcal{I}_{\mathbf{k}, n}\left(-\eta_{1} \mathbf{P}_{\varphi}\right)\right]\right\} .
\end{aligned}
$$

Because the vector boson $\varphi^{\mu}$ is transverse, the $\mu=0$ component and the component parallel to $\mathbf{P}_{\varphi}$ do not contribute to the amplitude $\mathcal{M}_{\mathbf{k} \rightarrow n}=\epsilon_{\mu} \mathcal{M}_{\mathbf{k} \rightarrow n}^{\mu}$. Dropping those components, we obtain $\mathcal{M}_{\mathbf{k} \rightarrow n}^{j} \rightarrow \tilde{\mathcal{M}}_{\mathbf{k} \rightarrow n}^{j}$. In the rest frame, $\mathbf{K}=0$, and for capture in 
the ground state $\{100\}$, using eq. (F.41) for the $\mathcal{J}_{\mathbf{k},\{100\}}$ integrals, and keeping only the leading term, we find

$$
\tilde{\mathcal{M}}_{\mathbf{k} \rightarrow n}^{j}=-2 g \sqrt{2 \mu} \mathcal{R}(\zeta) \frac{k^{j} \sin \theta}{k^{3 / 2}}\left(\frac{c_{1}}{\eta_{1}}-\frac{c_{2}}{\eta_{2}}\right),
$$

where the $\sin \theta$ factor arises from the projection of $\mathbf{k}$ on the plane vertical to $\mathbf{P}_{\varphi}$, and $\mathcal{R}(\zeta)$ is defined in eq. (F.36). (Recall that $c_{1} c_{2}<0$ for an attractive potential.) Note that the partial wave decomposition of the $\theta$-dependent factor of eq. (5.55) is

$$
\sin \theta=\sqrt{1-\cos ^{2} \theta}=\frac{\pi}{4} P_{0}(\cos \theta)-\frac{5 \pi}{2^{5}} P_{2}(\cos \theta)-\frac{3^{2} \pi}{2^{8}} P_{4}(\cos \theta)+\ldots
$$

The sum over the vector-boson polarisations is

$$
\sum_{\epsilon}\left|\epsilon_{\mu} \mathcal{M}_{\mathbf{k} \rightarrow n}^{\mu}\right|^{2}=\mathcal{M}_{\mathbf{k} \rightarrow n}^{\mu *} \mathcal{M}_{\mathbf{k} \rightarrow n}^{\nu} \sum_{\epsilon} \epsilon_{\mu}^{*} \epsilon_{\nu}=-\mathcal{M}_{\mathbf{k} \rightarrow n}^{\mu *} \mathcal{M}_{\mathbf{k} \rightarrow n}^{\nu} g_{\mu \nu}=\tilde{\mathcal{M}}_{\mathbf{k} \rightarrow n}^{j *} \tilde{\mathcal{M}}_{\mathbf{k} \rightarrow n}^{j} .
$$

In eq. (5.57), the contribution of the $\mu=0$ and $\nu=0$ components cancels the contribution from the component of $\mathcal{M}$ that is parallel to $\mathbf{P}_{\varphi}$, yielding the final result. Using eqs. (5.55) and (F.37), we obtain

$$
\sum_{\epsilon}\left|\epsilon_{\mu} \mathcal{M}_{\mathbf{k} \rightarrow n}^{\mu}\right|^{2}=\left[\frac{\left(\eta_{2} c_{1}-\eta_{1} c_{1}\right)^{2}}{-c_{1} c_{2}}\right] \frac{2^{12} \pi^{3}}{\eta_{1}^{2} \eta_{2}^{2}} \frac{\zeta^{7}}{\left(1+\zeta^{2}\right)^{3}} \frac{e^{-4 \zeta \operatorname{arccot} \zeta}}{1-e^{-2 \pi \zeta}} \sin ^{2} \theta .
$$

Note that for $c_{1}=-c_{2}=1$, which includes the case of a particle-antiparticle pair, the factor in the square brackets in the above expression becomes $\left[\left(\eta_{2} c_{1}-\eta_{1} c_{2}\right)^{2} /\left(-c_{1} c_{2}\right)\right]=1$.

Using eqs. (3.43) and (5.58), we find the unpolarised cross-section for radiative capture to the ground state to be

$$
\sigma_{\mathrm{BSF}}^{\{100\}} v_{\mathrm{rel}}=\left[\frac{\left(\eta_{2} c_{1}-\eta_{1} c_{2}\right)^{2}}{-c_{1} c_{2}}\right] \frac{2^{8} \pi^{2} \alpha^{2}}{3 \mu^{2}} \frac{\zeta^{5}}{\left(1+\zeta^{2}\right)^{2}} \frac{e^{-4 \zeta \operatorname{arccot} \zeta}}{1-e^{-2 \pi \zeta}} .
$$

This result agrees with ref. [63] (see eq. (75.6)), and is smaller than that of ref. [64] by a factor of 2 . At $\zeta \gg 1$, eq. (5.59) becomes

$$
\sigma_{\mathrm{BSF}}^{\{100\}} v_{\mathrm{rel}} \simeq\left[\frac{\left(\eta_{2} c_{1}-\eta_{1} c_{2}\right)^{2}}{-c_{1} c_{2}}\right] \frac{2^{8} \pi^{2} \alpha^{2}}{3 e^{4} \mu^{2}} \zeta
$$

The contribution from the $J=0$ partial wave is $\sigma_{\mathrm{BSF}, J=0}^{\{100\}}=(\pi / 4)^{2} \sigma_{\mathrm{BSF}}^{\{100\}}$ (cf. eq. (5.56)). The unitarity limit on the $s$-wave inelastic cross-section, given in eq. (3.48), is realised for $\alpha=\alpha_{\text {uni }}$, with

$$
\alpha_{\text {uni }} \approx 0.69\left[\frac{-c_{1} c_{2}}{\left(\eta_{2} c_{1}-\eta_{1} c_{2}\right)^{2}}\right]^{1 / 3}
$$

\subsubsection{Annihilation vs bound-state formation for particle-antiparticle pairs}

In the non-relativistic regime, the annihilation of a particle-antiparticle pair into two vector mediators, $\chi \chi^{\dagger} \rightarrow \varphi \varphi$, is $s$-wave dominated, with the perturbative unpolarised cross-section times relative velocity being $\sigma_{0}=\pi \alpha^{2} /\left(2 \mu^{2}\right)$. From eqs. (4.24) and (4.27), we find

$$
\sigma_{\text {ann }} v_{\text {rel }}=\sigma_{0}\left|\phi_{\mathbf{k}}(0)\right|^{2}=\frac{\pi \alpha^{2}}{2 \mu^{2}} \frac{2 \pi \zeta}{1-e^{-2 \pi \zeta}},
$$

where here $\mu=m_{\chi} / 2=m / 4$, with $m_{\chi}$ being the mass of the annihilating particles. 
We may now compare the BSF and annihilation cross-sections:

$$
\frac{\sigma_{\mathrm{BSF}}^{\{100\}}}{\sigma_{\mathrm{ann}}} \simeq \frac{2^{8} \zeta^{4} e^{-4 \zeta \operatorname{arccot} \zeta}}{3\left(1+\zeta^{2}\right)^{2}} .
$$

While at $\zeta<1 \mathrm{BSF}$ is negligible, at $\zeta \gtrsim 1.11$ the contribution of BSF to the inelastic scattering dominates over annihilation. At $\zeta \gg 1, \sigma_{\mathrm{BSF}}^{\{100\}} / \sigma_{\mathrm{ann}} \simeq 1.56 .{ }^{17}$ We compare $\sigma_{\text {ann }}$ and $\sigma_{\mathrm{BSF}}^{\{100\}}$ in figure 1 .

\subsubsection{Particle-antiparticle bound-state decay rate}

From eq. (4.25), we find the unpolarised decay rate of a particle-antiparticle bound state into two vector mediators to be

$$
\Gamma_{\{100\} \rightarrow \varphi \varphi} \simeq \sigma_{0}\left|\psi_{100}(0)\right|^{2}=\frac{\pi \alpha^{2}}{2 \mu^{2}} \frac{\kappa^{3}}{\pi}=\frac{\mu \alpha^{5}}{2} .
$$

\section{Discussion}

The formation of bound states affects the phenomenology of dark matter in a variety of ways. Computing the rates for bound-state formation and other related processes is essential in calculating the cosmology of DM and accurately estimating the expected DM signals and detection prospects.

In the non-relativistic regime, the formation of bound states is enhanced by the Sommerfeld effect. The Sommerfeld effect has already been incorporated in computations of the DM annihilation rate, in the context of various theories, and has been shown to have important phenomenological implications. Besides enhancing the total DM annihilation rate, it may also modify - depending on the nature of the DM interactions - the relative strength of the various annihilation channels, thus changing the spectrum of the annihilation products [73]. Our results demonstrate that, for particle-antiparticle pairs or pairs of self-conjugate particles, the radiative formation of bound states can be faster than annihilation, in the entire regime where the Sommerfeld effect is important. This suggests that bound-state formation and decay may affect the annihilation signals of symmetric thermal-relic dark matter, as well as its relic abundance, well beyond the experimental uncertainty in the DM density [43]. Bound-state dynamics should then be incorporated in any relevant analyses.

The importance of this point is underscored by present experimental results, which strongly constrain sub-TeV DM with electroweak interactions, and thus motivate investigations in the multi- $\mathrm{TeV}$ regime. As is well known, for symmetric (or self-conjugate) thermal-relic DM heavier than a few TeV, including WIMP DM, the Sommerfeld effect is important, both in the determination of the relic abundance and in the estimation of the expected indirect-detection signals. For the indirect detection of hidden-sector DM, the Sommerfeld effect - and therefore the formation of bound states - can be important even for lower DM masses.

\footnotetext{
${ }^{17}$ For a fermionic particle-antiparticle pair coupled to a gauged $\mathrm{U}(1), \sigma_{0} \simeq \pi \alpha^{2} /\left(4 \mu^{2}\right)$. Then, at $\zeta \gg 1$, the importance of BSF relative to annihilation is even greater, with $\sigma_{\mathrm{BSF}}^{\{100\}} / \sigma_{\mathrm{ann}} \simeq 3.1$.
} 
Asymmetric dark matter can couple even more strongly to light force mediators than symmetric DM; indeed, in the presence of a particle-antiparticle asymmetry, the very efficient annihilation which such a coupling would imply, cannot destroy the DM relic abundance. It follows that, for a much larger range of masses, asymmetric DM may efficiently form stable bound states in the early universe. This has important implications for its phenomenology. On one hand, the formation of bound states typically curtails the DM self-interactions and hastens the kinetic decoupling of DM from dark radiation in the early universe; consequently, it regulates the potential effect of the DM dynamics on the galactic structure. On the other hand, DM may participate in a variety of radiative processes inside haloes, such as excitations and de-excitations of bound states, or outright formation of bound states. In addition, the scattering of DM on nucleons may involve a variety of interactions, including both elastic and inelastic processes. This interplay between cosmology and the fundamental interactions of the DM constituents, determines all manifestations of DM today, and can be calculated only with precise knowledge of the rates governing bound-state-related processes.

In this work, we established a field-theoretic framework for computing rates for processes involving bound states. This framework can be employed in future investigations of related effects, in a variety of theories. In particular, the computation of bound-state formation rates in theories which involve non-Abelian interactions - including the electroweak theory of the Standard Model - necessitates adopting a field-theoretic formalism. Moreover, this framework allows for systematic expansions in the interaction strength and in the momentum exchange between the interacting degrees of freedom; these higher-order corrections are important when the leading-order terms cancel, as was explicitly shown in our computations.

The significance of long-range interactions - and therefore, the importance of comprehending their implications - is affirmed by unitarity. Unitarity sets an upper bound on the partial-wave inelastic cross-section, $\left(\sigma_{\text {inel }, J}\right)_{\max }$, shown in eq. (3.48). This, in turn, yields an upper bound on the mass of thermal-relic DM [43, 71]. Notably, the velocity dependence of $\left(\sigma_{\text {inel, } J}\right)_{\max }$ suggests that the unitarity bound can be realised only if the underlying interactions are long-ranged [43]. However, in the presence of long-range interactions, the formation of bound states may be the dominant inelastic process, as shown in the present work. The realisation of the unitarity bound, and its phenomenological implications, are thus largely determined by the dynamics of bound states, which should be fully incorporated in any related study. For example, the DM self-destruction in the early universe via bound-state formation and decay involves an interplay between capture, disassociation and decay processes that is absent in the case of direct annihilation into radiation [43].

Moreover, our results show that in the large-coupling (or low-velocity) regime, the dominant inelastic channel often belongs to a higher partial wave than usually assumed. Since the unitarity bound on higher partial waves is more relaxed, this implies that thermalrelic DM may be significantly heavier than previously estimated.

Lastly, using the computed bound-state-formation cross-sections, we may estimate the interaction strength for which the unitarity bound is seemingly realised. Our leading-order 
computations show that this is at $\alpha \sim 0.5$, i.e. well below what is often considered to be the perturbativity limit, $\alpha \sim \pi$ or $4 \pi$.

\section{Acknowledgments}

We thank Paul Hoyer for very helpful discussions. This work was supported by the Netherlands Foundation for Fundamental Research of Matter (FOM) and the Netherlands Organisation for Scientific Research (NWO).

\section{A Bethe-Salpeter wavefunctions: summary of definitions}

We summarise the definitions of the Bethe-Salpeter wavefunctions and their Fourier transforms. Following section 2.1, we find

$$
\begin{aligned}
& \Psi_{\mathbf{Q}, n}\left(x_{1}, x_{2}\right)=e^{-i Q X} \Psi_{\mathbf{Q}, n}(x), \\
& \Psi_{\mathbf{Q}, n}^{\star}\left(x_{1}, x_{2}\right)=e^{i Q X} \Psi_{\mathbf{Q}, n}^{\star}(x), \\
& \Phi_{\mathbf{Q}, \mathbf{q}}\left(x_{1}, x_{2}\right)=e^{-i Q X} \Phi_{\mathbf{Q}, \mathbf{q}}(x), \\
& \Phi_{\mathbf{Q}, \mathbf{q}}^{\star}\left(x_{1}, x_{2}\right)=e^{i Q X} \Phi_{\mathbf{Q}, \mathbf{q}}^{\star}(x),
\end{aligned}
$$

with $Q^{0}=\omega_{n}(\mathbf{Q})$ and $Q^{0}=\omega_{\mathbf{Q}, \mathbf{q}}$ being the energies of $\left|\mathcal{B}_{\mathbf{Q}, n}\right\rangle$ and $\left|\mathcal{U}_{\mathbf{Q}, \mathbf{q}}\right\rangle$, and where we defined

$$
\begin{aligned}
& \Psi_{\mathbf{Q}, n}(x) \equiv\left\langle\Omega\left|T \chi_{1}\left(\eta_{2} x\right) \chi_{2}\left(-\eta_{1} x\right)\right| \mathcal{B}_{\mathbf{Q}, n}\right\rangle, \\
& \Psi_{\mathbf{Q}, n}^{\star}(x) \equiv\left\langle\mathcal{B}_{\mathbf{Q}, n}\left|T \chi_{1}^{\dagger}\left(\eta_{2} x\right) \chi_{2}^{\dagger}\left(-\eta_{1} x\right)\right| \Omega\right\rangle, \\
& \Phi_{\mathbf{Q}, \mathbf{q}}(x) \equiv\left\langle\Omega\left|T \chi_{1}\left(\eta_{2} x\right) \chi_{2}\left(-\eta_{1} x\right)\right| \mathcal{U}_{\mathbf{Q}, \mathbf{q}}\right\rangle, \\
& \Phi_{\mathbf{Q}, \mathbf{q}}^{\star}(x) \equiv\left\langle\mathcal{U}_{\mathbf{Q}, \mathbf{q}}\left|T \chi_{1}^{\dagger}\left(\eta_{2} x\right) \chi_{2}^{\dagger}\left(-\eta_{1} x\right)\right| \Omega\right\rangle .
\end{aligned}
$$

The Fourier transforms are

$$
\begin{aligned}
\Psi_{\mathbf{Q}, n}(x) & \equiv \int \frac{d^{4} p}{(2 \pi)^{4}} \tilde{\Psi}_{\mathbf{Q}, n}(p) e^{-i p x}, & \tilde{\Psi}_{\mathbf{Q}, n}(p) & \equiv \int d^{4} x \Psi_{\mathbf{Q}, n}(x) e^{i p x} \\
\Psi_{\mathbf{Q}, n}^{\star}(x) & \equiv \int \frac{d^{4} p}{(2 \pi)^{4}} \tilde{\Psi}_{\mathbf{Q}, n}^{\star}(p) e^{i p x}, & \tilde{\Psi}_{\mathbf{Q}, n}^{\star}(p) & \equiv \int d^{4} x \Psi_{\mathbf{Q}, n}^{\star}(x) e^{-i p x}, \\
\Phi_{\mathbf{Q}, \mathbf{q}}(x) & \equiv \int \frac{d^{4} p}{(2 \pi)^{4}} \tilde{\Phi}_{\mathbf{Q}, \mathbf{q}}(p) e^{-i p x}, & \tilde{\Phi}_{\mathbf{Q}, \mathbf{q}}(p) & \equiv \int d^{4} x \Phi_{\mathbf{Q}, \mathbf{q}}(x) e^{i p x} \\
\Phi_{\mathbf{Q}, \mathbf{q}}^{\star}(x) & \equiv \int \frac{d^{4} p}{(2 \pi)^{4}} \tilde{\Phi}_{\mathbf{Q}, \mathbf{q}}(p) e^{i p x}, & \tilde{\Phi}_{\mathbf{Q}, \mathbf{q}}^{\star}(p) & \equiv \int d^{4} x \Phi_{\mathbf{Q}, \mathbf{q}}^{\star}(x) e^{-i p x}
\end{aligned}
$$

\section{B Calculation of $h\left(x^{0}\right)$}

As in eq. (2.9), we define

$$
\begin{array}{ll}
x_{1}=X+\eta_{2} x, & x_{2}=X-\eta_{1} x, \\
y_{1}=Y+\eta_{2} y, & y_{2}=Y-\eta_{1} y .
\end{array}
$$


Then

$$
\begin{aligned}
& \min \left(x_{1}^{0}, x_{2}^{0}\right)-\max \left(y_{1}^{0}, y_{2}^{0}\right)= \\
& =\min \left(X^{0}+\eta_{2} x^{0}, X^{0}-\eta_{1} x^{0}\right)-\max \left(Y^{0}+\eta_{2} y^{0}, Y^{0}-\eta_{1} y^{0}\right) \\
& =X^{0}+\min \left(\eta_{2} x^{0},-\eta_{1} x^{0}\right)-Y^{0}-\max \left(\eta_{2} y^{0},-\eta_{1} y^{0}\right)
\end{aligned}
$$

where, as defined in eq. (2.36),

$$
h_{ \pm}\left(x^{0}\right) \equiv \frac{1}{2}\left(\eta_{2}-\eta_{1}\right) x^{0} \pm \frac{1}{2}\left|x^{0}\right| .
$$

\section{Calculation of $\mathcal{S}_{0}(\mathrm{p} ; P), \mathcal{S}(t ; \mathrm{p} ; P)$ and $\mathcal{S}_{1,2}(t ; \mathrm{p} ; P)$}

Here we calculate the integrals

$$
\begin{aligned}
\mathcal{S}_{0}(\mathbf{p} ; P) & \equiv \int \frac{d p^{0}}{2 \pi} \tilde{S}_{1}\left(\eta_{1} P+p\right) \tilde{S}_{2}\left(\eta_{2} P-p\right), \\
\mathcal{S}(t ; \mathbf{p} ; P) & \equiv \int \frac{d p^{0}}{2 \pi} \tilde{S}_{1}\left(\eta_{1} P+p\right) \tilde{S}_{2}\left(\eta_{2} P-p\right) e^{i p^{0} t}, \\
\mathcal{S}_{1}(t ; \mathbf{p} ; P) & \equiv \int \frac{d p^{0}}{2 \pi} \tilde{S}_{1}\left(\eta_{1} P+p\right) e^{-i p^{0} t}, \\
\mathcal{S}_{2}(t ; \mathbf{p} ; P) & \equiv \int \frac{d p^{0}}{2 \pi} \tilde{S}_{2}\left(\eta_{2} P-p\right) e^{-i p^{0} t} .
\end{aligned}
$$

(Note the different sign in the exponential between the definitions (C.2) and (C.3), (C.4), chosen so for later convenience.)

We shall use the perturbative propagators

$$
\tilde{S}_{1}\left(p_{1}\right)=\frac{i}{p_{1}^{2}-m_{1}^{2}+i \epsilon}, \quad \tilde{S}_{2}\left(p_{2}\right)=\frac{i}{p_{2}^{2}-m_{2}^{2}+i \epsilon},
$$

and we define $E_{1}(\mathbf{p} ; \mathbf{P})=\sqrt{\left(\eta_{1} \mathbf{P}+\mathbf{p}\right)^{2}+m_{1}^{2}}$ and $E_{2}(\mathbf{p} ; \mathbf{P})=\sqrt{\left(\eta_{2} \mathbf{P}-\mathbf{p}\right)^{2}+m_{2}^{2}}$. For convenience, we also define

$$
\begin{aligned}
& \rho_{1}(\mathbf{p} ; P) \equiv \eta_{1} P^{0}-E_{1}(\mathbf{p} ; \mathbf{P}), \\
& \rho_{2}(\mathbf{p} ; P) \equiv \eta_{2} P^{0}-E_{2}(\mathbf{p} ; \mathbf{P}), \\
& \sigma_{1}(\mathbf{p} ; P) \equiv \eta_{1} P^{0}+E_{1}(\mathbf{p} ; \mathbf{P}), \\
& \sigma_{2}(\mathbf{p} ; P) \equiv \eta_{2} P^{0}+E_{2}(\mathbf{p} ; \mathbf{P}) .
\end{aligned}
$$


We first consider the integral (C.2),

$$
\begin{aligned}
& \mathcal{S}(t ; \mathbf{p} ; P)=\int \frac{d p^{0}}{2 \pi} \frac{i}{\left[\eta_{1} P^{0}+p^{0}-E_{1}(\mathbf{p} ; \mathbf{P})+i \epsilon\right]\left[\eta_{1} P^{0}+p^{0}+E_{1}(\mathbf{p} ; \mathbf{P})-i \epsilon\right]} \\
& \times \frac{i}{\left[\eta_{2} P^{0}-p^{0}+E_{2}(\mathbf{p} ; \mathbf{P})-i \epsilon\right]\left[\eta_{2} P^{0}-p^{0}-E_{2}(\mathbf{p} ; \mathbf{P})+i \epsilon\right]} e^{i p^{0} t} .
\end{aligned}
$$

We may evaluate $\mathcal{S}(t ; \mathbf{p} ; P)$ by closing the $p^{0}$ contour above and below the real axis for $t>0$ and $t<0$, respectively. The poles that contribute in each case are

$$
\begin{array}{lll}
t>0: & p^{0}=-\eta_{1} P^{0}-E_{1}(\mathbf{p} ; \mathbf{P})+i \epsilon, & p^{0}=\eta_{2} P^{0}-E_{2}(\mathbf{p} ; \mathbf{P})+i \epsilon, \\
t<0: & p^{0}=-\eta_{1} P^{0}+E_{1}(\mathbf{p} ; \mathbf{P})-i \epsilon, & p^{0}=\eta_{2} P^{0}+E_{2}(\mathbf{p} ; \mathbf{P})-i \epsilon .
\end{array}
$$

Then

$$
\mathcal{S}(t ; \mathbf{p} ; P)= \begin{cases}i\left[\frac{e^{-i \rho_{1}(\mathbf{p} ; P) t}}{2 E_{1}\left[\left(P^{0}-E_{1}\right)^{2}-E_{2}^{2}\right]}+\frac{e^{i \sigma_{2}(\mathbf{p} ; P) t}}{2 E_{2}\left[\left(P^{0}+E_{2}\right)^{2}-E_{1}^{2}\right]}\right], & \text { for } t<0, \\ i\left[\frac{e^{-i \sigma_{1}(\mathbf{p} ; P) t}}{2 E_{1}\left[\left(P^{0}+E_{1}\right)^{2}-E_{2}^{2}\right]}+\frac{e^{i \rho_{2}(\mathbf{p} ; P) t}}{2 E_{2}\left[\left(P^{0}-E_{2}\right)^{2}-E_{1}^{2}\right]}\right], & \text { for } t>0,\end{cases}
$$

and

$$
\mathcal{S}_{0}(\mathbf{p} ; P)=\mathcal{S}(t=0 ; \mathbf{p} ; P)=\frac{i\left(E_{1}+E_{2}\right)}{2 E_{1} E_{2}\left[\left(P^{0}\right)^{2}-\left(E_{1}+E_{2}\right)^{2}\right]} .
$$

It will be useful to rewrite eq. (C.13), using eq. (C.14), as follows

$$
\begin{aligned}
& \mathcal{S}(t ; \mathbf{p} ; P)=\frac{\mathcal{S}_{0}(\mathbf{p} ; P)}{E_{1}+E_{2}} \times \\
& \quad \times \begin{cases}{\left[\left(\frac{P^{0}+E_{1}+E_{2}}{P^{0}-E_{1}+E_{2}}\right) E_{2} e^{-i \rho_{1}(\mathbf{p} ; P) t}+\left(\frac{P^{0}-E_{1}-E_{2}}{P^{0}-E_{1}+E_{2}}\right) E_{1} e^{i \sigma_{2}(\mathbf{p} ; P) t}\right],} & \text { for } t<0, \\
{\left[\left(\frac{P^{0}-E_{1}-E_{2}}{P^{0}+E_{1}-E_{2}}\right) E_{2} e^{-i \sigma_{1}(\mathbf{p} ; P) t}+\left(\frac{P^{0}+E_{1}+E_{2}}{P^{0}+E_{1}-E_{2}}\right) E_{1} e^{i \rho_{2}(\mathbf{p} ; P) t}\right],} & \text { for } t>0 .\end{cases}
\end{aligned}
$$

(Note that in (C.13)-(C.15), it is implied that $E_{1}$ and $E_{2}$ come with the arguments $(\mathbf{p} ; \mathbf{P})$.)

Similarly to the above, we find

$$
\mathcal{S}_{1}(t ; \mathbf{p} ; P)= \begin{cases}\frac{e^{i \sigma_{1}(\mathbf{p} ; P) t}}{2 E_{1}(\mathbf{p} ; \mathbf{P})}, & \text { for } t<0, \\ \frac{e^{i \rho_{1}(\mathbf{p} ; P) t}}{2 E_{1}(\mathbf{p} ; \mathbf{P})}, & \text { for } t>0\end{cases}
$$

and

$$
\mathcal{S}_{2}(t ; \mathbf{p} ; P)= \begin{cases}\frac{e^{-i \rho_{2}(\mathbf{p} ; P) t}}{2 E_{2}(\mathbf{p} ; \mathbf{P})}, & \text { for } t<0, \\ \frac{e^{-i \sigma_{2}(\mathbf{p} ; P) t}}{2 E_{2}(\mathbf{p} ; \mathbf{P})}, & \text { for } t>0\end{cases}
$$


Non-relativistic approximation. In the non-relativistic regime, $\mathbf{P}, \mathbf{p} \ll P^{0}, m_{1}, m_{2}$. Then

$$
\begin{aligned}
E_{1}(\mathbf{p} ; \mathbf{P}) & \simeq \eta_{1}\left(m+\frac{\mathbf{P}^{2}}{2 m}\right)+\frac{\mathbf{P} \cdot \mathbf{p}}{m}+\frac{\mathbf{p}^{2}}{2 m_{1}} \\
E_{2}(\mathbf{p} ; \mathbf{P}) & \simeq \eta_{2}\left(m+\frac{\mathbf{P}^{2}}{2 m}\right)-\frac{\mathbf{P} \cdot \mathbf{p}}{m}+\frac{\mathbf{p}^{2}}{2 m_{2}} \\
E_{1}(\mathbf{p} ; \mathbf{P})+E_{2}(\mathbf{p} ; \mathbf{P}) & \simeq m+\frac{\mathbf{P}^{2}}{2 m}+\frac{\mathbf{p}^{2}}{2 \mu}
\end{aligned}
$$

Note that in the last expression, the cancellation of the mixed terms proportional to $\mathbf{p} \cdot \mathbf{P}$, can be traced to eq. (2.11). This reflects the fact that in the non-relativistic regime, the relative motion can be separated from the motion of the CM. For convenience, we set

$$
P^{0}=m+\frac{\mathbf{P}^{2}}{2 m}+\mathcal{E}
$$

With these approximations, eq. (C.14) becomes

$$
\mathcal{S}_{0}(\mathbf{p} ; P) \simeq-\frac{1}{i 4 m \mu\left(P^{0}-m-\frac{\mathbf{p}^{2}}{2 m}-\frac{\mathbf{p}^{2}}{2 \mu}\right)}=-\frac{1}{i 4 m \mu\left(\mathcal{E}-\frac{\mathbf{p}^{2}}{2 \mu}\right)} .
$$

\section{Partial-wave analysis for (co-)annihilation and decay processes}

We will prove the following two relations

$$
\int \frac{d^{3} q}{(2 \pi)^{3}} \tilde{\phi}_{\mathbf{k}}(\mathbf{q})|\mathbf{q}|^{\ell} P_{\ell}\left(\cos \theta_{\mathbf{q}, \mathbf{p}}\right)=\frac{(2 \ell+1) ! !}{4 \pi i^{\ell} \ell !}\left[\frac{d^{\ell}}{d r^{\ell}} \int d \Omega_{\mathbf{r}} P_{\ell}\left(\cos \theta_{\mathbf{p}, \mathbf{r}}\right) \phi_{\mathbf{k}}(\mathbf{r})\right]_{\mathbf{r}=0}
$$

and

$$
\begin{aligned}
\int d \Omega_{\mathbf{p}} P_{\ell^{\prime}}\left(\cos \theta_{\mathbf{p}}\right) \int \frac{d^{3} q}{(2 \pi)^{3}} & \tilde{\phi}_{\mathbf{k}}(\mathbf{q})|\mathbf{q}|^{\ell} P_{\ell}\left(\cos \theta_{\mathbf{q}, \mathbf{p}}\right)= \\
& =\delta_{\ell \ell^{\prime}} \frac{(2 \ell+1) ! !}{i^{\ell}(2 \ell+1) \ell !}\left[\frac{d^{\ell}}{d r^{\ell}} \int d \Omega_{\mathbf{r}} P_{\ell}\left(\cos \theta_{\mathbf{r}}\right) \phi_{\mathbf{k}}(\mathbf{r})\right]_{\mathbf{r}=0} .
\end{aligned}
$$

We shall use the addition theorem of spherical harmonics,

$$
P_{\ell}(\hat{\mathbf{x}} \cdot \hat{\mathbf{y}})=\frac{4 \pi}{2 \ell+1} \sum_{m=-\ell}^{\ell} Y_{\ell m}^{*}\left(\Omega_{\mathbf{x}}\right) Y_{\ell m}\left(\Omega_{\mathbf{y}}\right)
$$

where $\hat{\mathbf{x}}, \hat{\mathbf{y}}$ are unit vectors, and $Y_{\ell m}$ are the spherical harmonics. From eq. (D.3) and the orthonormality of $Y_{\ell m}$, it follows that

$$
\int d \Omega_{\mathbf{x}} Y_{\ell m}\left(\Omega_{\mathbf{x}}\right) P_{\ell^{\prime}}\left(\cos \theta_{\mathbf{x}, \mathbf{y}}\right)=\frac{4 \pi}{2 \ell+1} Y_{\ell m}\left(\Omega_{\mathbf{y}}\right) \delta_{\ell \ell^{\prime}}
$$

We will also need the expansion

$$
e^{i \mathbf{q} \cdot \mathbf{r}}=\sum_{\ell=0}^{\infty}(2 \ell+1) i^{\ell} j_{\ell}(q r) P_{\ell}\left(\cos \theta_{\mathbf{q}, \mathbf{r}}\right)
$$


where $j_{\ell}$ is the spherical Bessel function, which satisfies

$$
\left.\frac{d^{\ell} j_{\ell}(x)}{d x^{\ell}}\right|_{x=0}=\frac{\ell !}{(2 \ell+1) ! !} .
$$

We begin with the right side of eq. (D.1). Using eqs. (D.3)-(D.6) and the Fourier transform of $\tilde{\phi}_{\mathbf{k}}(\mathbf{q})$, we find

$$
\begin{aligned}
& {\left[\frac{d^{\ell}}{d r^{\ell}} \int d \Omega_{\mathbf{r}} P_{\ell}\left(\cos \theta_{\mathbf{p}, \mathbf{r}}\right) \phi_{\mathbf{k}}(\mathbf{r})\right]_{\mathbf{r}=0}=} \\
& \quad=\frac{4 \pi}{2 \ell+1} \sum_{m=-\ell}^{\ell} Y_{\ell m}^{*}\left(\Omega_{\mathbf{p}}\right) \int \frac{d^{3} q}{(2 \pi)^{3}} \tilde{\phi}_{\mathbf{k}}(\mathbf{q})\left[\frac{d^{\ell}}{d r^{\ell}} \int d \Omega_{\mathbf{r}} Y_{\ell m}\left(\Omega_{\mathbf{r}}\right) e^{i \mathbf{q} \cdot \mathbf{r}}\right]_{\mathbf{r}=0} \\
& =\frac{4 \pi}{2 \ell+1} \sum_{m=-\ell}^{\ell} Y_{\ell m}^{*}\left(\Omega_{\mathbf{p}}\right) \int \frac{d^{3} q}{(2 \pi)^{3}} \tilde{\phi}_{\mathbf{k}}(\mathbf{q}) \\
& \quad \times \sum_{\ell^{\prime}=0}^{\infty}\left(2 \ell^{\prime}+1\right) i^{\ell^{\prime}}\left[\frac{d^{\ell}}{d r^{\ell}} j_{\ell^{\prime}}(q r)\right]_{\mathbf{r}=0} \int d \Omega_{\mathbf{r}} Y_{\ell m}\left(\Omega_{\mathbf{r}}\right) P_{\ell^{\prime}}\left(\cos \theta_{\mathbf{q}, \mathbf{r}}\right) \\
& =\left(\frac{4 \pi}{2 \ell+1}\right)^{2} \sum_{m=-\ell}^{\ell} Y_{\ell m}^{*}\left(\Omega_{\mathbf{p}}\right) \int \frac{d^{3} q}{(2 \pi)^{3}} \tilde{\phi}_{\mathbf{k}}(\mathbf{q})|\mathbf{q}|^{\ell}(2 \ell+1) i^{\ell}\left[\frac{d^{\ell} j_{\ell}(x)}{d x^{\ell}}\right]_{x=0} Y_{\ell m}\left(\Omega_{\mathbf{q}}\right) \\
& =\frac{4 \pi i^{\ell} \ell !}{(2 \ell+1) ! !} \int \frac{d^{3} q}{(2 \pi)^{3}} \tilde{\phi}_{\mathbf{k}}(\mathbf{q})|\mathbf{q}|^{\ell} P_{\ell}\left(\cos \theta_{\mathbf{p}, \mathbf{q}}\right)
\end{aligned}
$$

This proves eq. (D.1).

Acting on the left side of eq. (D.1) with $\int d \Omega_{\mathbf{p}} P_{\ell^{\prime}}\left(\cos \theta_{\mathbf{p}}\right)$, we find

$$
\begin{aligned}
\int d \Omega_{\mathbf{p}} & P_{\ell^{\prime}}\left(\cos \theta_{\mathbf{p}}\right) \int \frac{d^{3} q}{(2 \pi)^{3}} \tilde{\phi}_{\mathbf{k}}(\mathbf{q})|\mathbf{q}|^{\ell} P_{\ell}\left(\cos \theta_{\mathbf{q}, \mathbf{p}}\right)= \\
= & \frac{(2 \ell+1) ! !}{4 \pi i^{\ell} \ell !} \sqrt{\frac{4 \pi}{2 \ell+1}} \int d \Omega_{\mathbf{p}} Y_{\ell^{\prime}, 0}\left(\Omega_{\mathbf{p}}\right)\left[\frac{d^{\ell}}{d r^{\ell}} \int d \Omega_{\mathbf{r}} P_{\ell}\left(\cos \theta_{\mathbf{p}, \mathbf{r}}\right) \phi_{\mathbf{k}}(\mathbf{r})\right]_{\mathbf{r}=0} \\
= & \frac{(2 \ell+1) ! !}{4 \pi i^{\ell} \ell !}\left(\frac{4 \pi}{2 \ell+1}\right)^{3 / 2} \delta_{\ell \ell^{\prime}}\left[\frac{d^{\ell}}{d r^{\ell}} \int d \Omega_{\mathbf{r}} Y_{\ell, 0}\left(\Omega_{\mathbf{r}}\right) \phi_{\mathbf{k}}(\mathbf{r})\right]_{\mathbf{r}=0} \\
= & \delta_{\ell \ell^{\prime}} \frac{(2 \ell+1) ! !}{i^{\ell}(2 \ell+1) \ell !}\left[\frac{d^{\ell}}{d r^{\ell}} \int d \Omega_{\mathbf{r}} P_{\ell}\left(\cos \theta_{\mathbf{r}}\right) \phi_{\mathbf{k}}(\mathbf{r})\right]_{\mathbf{r}=0} .
\end{aligned}
$$

This proves eq. (D.2).

\section{E Integrals for the non-relativistic reduction of transition amplitudes}

The transition amplitudes of section 5 contain integrals of the following forms

$$
\begin{aligned}
& \Xi_{1}(\mathbf{q}, \mathbf{p} ; K, P) \equiv \int \frac{d q^{0}}{2 \pi} S(q ; K) \int \frac{d p^{0}}{2 \pi} \tilde{S}_{1}\left(\eta_{1} P+p\right)(2 \pi) \delta\left(q^{0}-p^{0}-\eta_{2} P_{\varphi}^{0}\right), \\
& \Xi_{2}(\mathbf{q}, \mathbf{p} ; K, P) \equiv \int \frac{d q^{0}}{2 \pi} S(q ; K) \int \frac{d p^{0}}{2 \pi} \tilde{S}_{2}\left(\eta_{2} P-p\right)(2 \pi) \delta\left(q^{0}-p^{0}+\eta_{1} P_{\varphi}^{0}\right) .
\end{aligned}
$$


We are interested in evaluating $\Xi_{1}(\mathbf{q}, \mathbf{p} ; K, P)$ at $\mathbf{q}-\mathbf{p}-\eta_{2} \mathbf{P}_{\varphi}=0$, and $\Xi_{2}(\mathbf{q}, \mathbf{p} ; K, P)$ at $\mathbf{q}-\mathbf{p}+\eta_{1} \mathbf{P}_{\varphi}=0$. To evaluate $\Xi_{1}$ and $\Xi_{2}$, we Fourier-transform the $\delta$-function, and use eqs. (C.15), (C.16) and (C.17).

For $\Xi_{1}$, we obtain:

$$
\begin{aligned}
\Xi_{1}(\mathbf{q}, \mathbf{p} ; K, P)= & \int_{-\infty}^{\infty} d t e^{-i \eta_{2} P_{\varphi}^{0} t} \int \frac{d q^{0}}{2 \pi} S(q ; K) e^{i q^{0} t} \int \frac{d p^{0}}{2 \pi} \tilde{S}_{1}\left(\eta_{1} P+p\right) e^{-i p^{0} t} \\
= & \int_{-\infty}^{0} d t e^{-i \eta_{2} P_{\varphi}^{0} t} \mathcal{S}(t ; \mathbf{q} ; K) \mathcal{S}_{1}(t ; \mathbf{p} ; P) \\
& +\int_{0}^{\infty} d t e^{-i \eta_{2} P_{\varphi}^{0} t} \mathcal{S}(t ; \mathbf{q} ; K) \mathcal{S}_{1}(t ; \mathbf{p} ; P) \\
= & \frac{\mathcal{S}_{0}(\mathbf{q} ; K)}{\left[E_{1}(\mathbf{q} ; \mathbf{K})+E_{2}(\mathbf{q} ; \mathbf{K})\right] 2 E_{1}(\mathbf{p} ; \mathbf{P})} \times\left[\Xi_{1}^{-}(\mathbf{q}, \mathbf{p} ; K, P)+\Xi_{1}^{+}(\mathbf{q}, \mathbf{p} ; K, P)\right],
\end{aligned}
$$

where

$$
\begin{aligned}
\Xi_{1}^{-}(\mathbf{q}, \mathbf{p} ; K, P)= & \frac{K^{0}+E_{1}(\mathbf{q} ; \mathbf{K})+E_{2}(\mathbf{q} ; \mathbf{K})}{K^{0}-E_{1}(\mathbf{q} ; \mathbf{K})+E_{2}(\mathbf{q} ; \mathbf{K})} E_{2}(\mathbf{q} ; \mathbf{K}) \int_{-\infty}^{0} d t e^{i\left[-\eta_{2} P_{\varphi}^{0}-\rho_{1}(\mathbf{q} ; K)+\sigma_{1}(\mathbf{p} ; P)\right] t} \\
& +\frac{K^{0}-E_{1}(\mathbf{q} ; \mathbf{K})-E_{2}(\mathbf{q} ; \mathbf{K})}{K^{0}-E_{1}(\mathbf{q} ; \mathbf{K})+E_{2}(\mathbf{q} ; \mathbf{K})} E_{1}(\mathbf{q} ; \mathbf{K}) \int_{-\infty}^{0} d t e^{i\left[-\eta_{2} P_{\varphi}^{0}+\sigma_{2}(\mathbf{q} ; K)+\sigma_{1}(\mathbf{p} ; P)\right] t}
\end{aligned}
$$

and

$$
\begin{aligned}
\Xi_{1}^{+}(\mathbf{q}, \mathbf{p} ; K, P)= & \frac{K^{0}-E_{1}(\mathbf{q} ; \mathbf{K})-E_{2}(\mathbf{q} ; \mathbf{K})}{K^{0}+E_{1}(\mathbf{q} ; \mathbf{K})-E_{2}(\mathbf{q} ; \mathbf{K})} E_{2}(\mathbf{q} ; \mathbf{K}) \int_{0}^{\infty} d t e^{i\left[-\eta_{2} P_{\varphi}^{0}-\sigma_{1}(\mathbf{q} ; K)+\rho_{1}(\mathbf{p} ; P)\right] t} \\
& +\frac{K^{0}+E_{1}(\mathbf{q} ; \mathbf{K})+E_{2}(\mathbf{q} ; \mathbf{K})}{K^{0}+E_{1}(\mathbf{q} ; \mathbf{K})-E_{2}(\mathbf{q} ; \mathbf{K})} E_{1}(\mathbf{q} ; \mathbf{K}) \int_{0}^{\infty} d t e^{i\left[-\eta_{2} P_{\varphi}^{0}+\rho_{2}(\mathbf{q} ; K)+\rho_{1}(\mathbf{p} ; P)\right] t}
\end{aligned}
$$

Using the definitions (C.6)-(C.9) and the overall energy-momentum conservation, $K=$ $P+P_{\varphi}$, we may simplify the phases appearing in the above integrals, as follows

$$
\begin{aligned}
& -\eta_{2} P_{\varphi}^{0}-\rho_{1}(\mathbf{q} ; K)+\sigma_{1}(\mathbf{p} ; P)=P^{0}-K^{0}+E_{1}(\mathbf{q} ; \mathbf{K})+E_{1}(\mathbf{p} ; \mathbf{P}), \\
& -\eta_{2} P_{\varphi}^{0}+\sigma_{2}(\mathbf{q} ; K)+\sigma_{1}(\mathbf{p} ; P)=P^{0}+E_{2}(\mathbf{q} ; \mathbf{K})+E_{1}(\mathbf{p} ; \mathbf{P}), \\
& -\eta_{2} P_{\varphi}^{0}-\sigma_{1}(\mathbf{q} ; K)+\rho_{1}(\mathbf{p} ; P)=P^{0}-K^{0}-E_{1}(\mathbf{q} ; \mathbf{K})-E_{1}(\mathbf{p} ; \mathbf{P}), \\
& -\eta_{2} P_{\varphi}^{0}+\rho_{2}(\mathbf{q} ; K)+\rho_{1}(\mathbf{p} ; P)=P^{0}-E_{2}(\mathbf{q} ; \mathbf{K})-E_{1}(\mathbf{p} ; \mathbf{P}) .
\end{aligned}
$$

Adding a small imaginary parts to the integration variable $t$, such that the integrals 
in (E.4), (E.5) converge, we find

$$
\begin{aligned}
\Xi_{1}^{-} & (\mathbf{q}, \mathbf{p} ; K, P)= \\
= & -\frac{i}{K^{0}-E_{1}(\mathbf{q} ; \mathbf{K})+E_{2}(\mathbf{q} ; \mathbf{K})} \\
& \times\left(\frac{\left[K^{0}+E_{1}(\mathbf{q} ; \mathbf{K})+E_{2}(\mathbf{q} ; \mathbf{K})\right] E_{2}(\mathbf{q} ; \mathbf{K})}{P^{0}-K^{0}+E_{1}(\mathbf{q} ; \mathbf{K})+E_{1}(\mathbf{p} ; \mathbf{P})}+\frac{\left[K^{0}-E_{1}(\mathbf{q} ; \mathbf{K})-E_{2}(\mathbf{q} ; \mathbf{K})\right] E_{1}(\mathbf{q} ; \mathbf{K})}{P^{0}+E_{2}(\mathbf{q} ; \mathbf{K})+E_{1}(\mathbf{p} ; \mathbf{P})}\right) \\
= & -i \frac{\left[P^{0}+E_{1}(\mathbf{p} ; \mathbf{P})+E_{1}(\mathbf{q} ; \mathbf{K})+E_{2}(\mathbf{q} ; \mathbf{K})\right]\left[E_{1}(\mathbf{q} ; \mathbf{K})+E_{2}(\mathbf{q} ; \mathbf{K})\right]-K^{0} E_{1}(\mathbf{q} ; K)}{\left[P^{0}-K^{0}+E_{1}(\mathbf{q} ; \mathbf{K})+E_{1}(\mathbf{p} ; \mathbf{P})\right]\left[P^{0}+E_{2}(\mathbf{q} ; \mathbf{K})+E_{1}(\mathbf{p} ; \mathbf{P})\right]},
\end{aligned}
$$

$$
\begin{aligned}
\Xi_{1}^{+} & (\mathbf{q}, \mathbf{p} ; K, P)= \\
= & \frac{i}{K^{0}+E_{1}(\mathbf{q} ; \mathbf{K})-E_{2}(\mathbf{q} ; \mathbf{K})} \\
& \times\left(\frac{\left[K^{0}-E_{1}(\mathbf{q} ; \mathbf{K})-E_{2}(\mathbf{q} ; \mathbf{K})\right] E_{2}(\mathbf{q} ; \mathbf{K})}{P^{0}-K^{0}-E_{1}(\mathbf{q} ; \mathbf{K})-E_{1}(\mathbf{p} ; \mathbf{P})}+\frac{\left[K^{0}+E_{1}(\mathbf{q} ; \mathbf{K})+E_{2}(\mathbf{q} ; \mathbf{K})\right] E_{1}(\mathbf{q} ; \mathbf{K})}{P^{0}-E_{2}(\mathbf{q} ; \mathbf{K})-E_{1}(\mathbf{p} ; \mathbf{P})}\right) \\
= & i \frac{\left[P^{0}-E_{1}(\mathbf{p} ; \mathbf{P})-E_{1}(\mathbf{q} ; \mathbf{K})-E_{2}(\mathbf{q} ; \mathbf{K})\right]\left[E_{1}(\mathbf{q} ; \mathbf{K})+E_{2}(\mathbf{q} ; \mathbf{K})\right]-K^{0} E_{1}(\mathbf{q} ; K)}{\left[P^{0}-K^{0}-E_{1}(\mathbf{q} ; \mathbf{K})-E_{1}(\mathbf{p} ; \mathbf{P})\right]\left[P^{0}-E_{2}(\mathbf{q} ; \mathbf{K})-E_{1}(\mathbf{p} ; \mathbf{P})\right]} .
\end{aligned}
$$

For $\Xi_{2}$, we obtain:

$$
\begin{aligned}
\Xi_{2}(\mathbf{q}, \mathbf{p} ; K, P)= & \int_{-\infty}^{\infty} d t e^{i \eta_{1} P_{\varphi}^{0} t} \int \frac{d q^{0}}{2 \pi} S(q ; K) e^{i q^{0} t} \int \frac{d p^{0}}{2 \pi} \tilde{S}_{2}\left(\eta_{1} P-p\right) e^{-i p^{0} t} f(p) \\
= & \int_{-\infty}^{0} d t e^{i \eta_{1} P_{\varphi}^{0} t} \mathcal{S}(t ; \mathbf{q} ; K) \mathcal{S}_{2}(t ; \mathbf{p} ; P) \\
& +\int_{0}^{\infty} d t e^{i \eta_{1} P_{\varphi}^{0} t} \mathcal{S}(t ; \mathbf{q} ; K) \mathcal{S}_{2}(t ; \mathbf{p} ; P) \\
= & \frac{\mathcal{S}_{0}(\mathbf{q} ; K)}{\left[E_{1}(\mathbf{q} ; \mathbf{K})+E_{2}(\mathbf{q} ; \mathbf{K})\right] 2 E_{2}(\mathbf{p} ; \mathbf{P})} \times\left[\Xi_{2}^{-}(\mathbf{q}, \mathbf{p} ; K, P)+\Xi_{2}^{+}(\mathbf{q}, \mathbf{p} ; K, P)\right],
\end{aligned}
$$

where

$$
\begin{aligned}
\Xi_{2}^{-}(\mathbf{q}, \mathbf{p} ; K, P) & =\frac{K^{0}+E_{1}(\mathbf{q} ; \mathbf{K})+E_{2}(\mathbf{q} ; \mathbf{K})}{K^{0}-E_{1}(\mathbf{q} ; \mathbf{K})+E_{2}(\mathbf{q} ; \mathbf{K})} E_{2}(\mathbf{q} ; \mathbf{K}) \int_{-\infty}^{0} d t e^{i\left[\eta_{1} P_{\varphi}^{0}-\rho_{1}(\mathbf{q} ; K)-\rho_{2}(\mathbf{p} ; P)\right] t} \\
& +\frac{K^{0}-E_{1}(\mathbf{q} ; \mathbf{K})-E_{2}(\mathbf{q} ; \mathbf{K})}{K^{0}-E_{1}(\mathbf{q} ; \mathbf{K})+E_{2}(\mathbf{q} ; \mathbf{K})} E_{1}(\mathbf{q} ; \mathbf{K}) \int_{-\infty}^{0} d t e^{i\left[\eta_{1} P_{\varphi}^{0}+\sigma_{2}(\mathbf{q} ; K)-\rho_{2}(\mathbf{p} ; P)\right] t},
\end{aligned}
$$

and

$$
\begin{aligned}
\Xi_{2}^{+}(\mathbf{q}, \mathbf{p} ; K, P) & =\frac{K^{0}-E_{1}(\mathbf{q} ; \mathbf{K})-E_{2}(\mathbf{q} ; \mathbf{K})}{K^{0}+E_{1}(\mathbf{q} ; \mathbf{K})-E_{2}(\mathbf{q} ; \mathbf{K})} E_{2}(\mathbf{q} ; \mathbf{K}) \int_{0}^{\infty} d t e^{i\left[\eta_{1} P_{\varphi}^{0}-\sigma_{1}(\mathbf{q} ; K)-\sigma_{2}(\mathbf{p} ; P)\right] t} \\
& +\frac{K^{0}+E_{1}(\mathbf{q} ; \mathbf{K})+E_{2}(\mathbf{q} ; \mathbf{K})}{K^{0}+E_{1}(\mathbf{q} ; \mathbf{K})-E_{2}(\mathbf{q} ; \mathbf{K})} E_{1}(\mathbf{q} ; \mathbf{K}) \int_{0}^{\infty} d t e^{i\left[\eta_{1} P_{\varphi}^{0}+\rho_{2}(\mathbf{q} ; K)-\sigma_{2}(\mathbf{p} ; P)\right] t}
\end{aligned}
$$


Using the definitions (C.6)-(C.9) and the overall energy-momentum conservation, $K=$ $P+P_{\varphi}$, we simplify the phases appearing in the above integrals, as follows

$$
\begin{aligned}
& \eta_{1} P_{\varphi}^{0}-\rho_{1}(\mathbf{q} ; K)-\rho_{2}(\mathbf{p} ; P)=-P^{0}+E_{1}(\mathbf{q} ; \mathbf{K})+E_{2}(\mathbf{p} ; \mathbf{P}) \\
& \eta_{1} P_{\varphi}^{0}+\sigma_{2}(\mathbf{q} ; K)-\rho_{2}(\mathbf{p} ; P)=-P^{0}+K^{0}+E_{2}(\mathbf{q} ; \mathbf{K})+E_{2}(\mathbf{p} ; \mathbf{P}), \\
& \eta_{1} P_{\varphi}^{0}-\sigma_{1}(\mathbf{q} ; K)-\sigma_{2}(\mathbf{p} ; P)=-P^{0}-E_{1}(\mathbf{q} ; \mathbf{K})-E_{2}(\mathbf{p} ; \mathbf{P}) \\
& \eta_{1} P_{\varphi}^{0}+\rho_{2}(\mathbf{q} ; K)-\sigma_{2}(\mathbf{p} ; P)=-P^{0}+K^{0}-E_{2}(\mathbf{q} ; \mathbf{K})-E_{2}(\mathbf{p} ; \mathbf{P}) .
\end{aligned}
$$

Adding a small imaginary parts to the integration variable $t$, as before, we find

$$
\begin{aligned}
\Xi_{2}^{-} & (\mathbf{q}, \mathbf{p} ; K, P)= \\
= & -\frac{i}{K^{0}-E_{1}(\mathbf{q} ; \mathbf{K})+E_{2}(\mathbf{q} ; \mathbf{K})} \\
& \times\left(\frac{\left[K^{0}+E_{1}(\mathbf{q} ; \mathbf{K})+E_{2}(\mathbf{q} ; \mathbf{K})\right] E_{2}(\mathbf{q} ; \mathbf{K})}{-P^{0}+E_{1}(\mathbf{q} ; \mathbf{K})+E_{2}(\mathbf{p} ; \mathbf{P})}+\frac{\left[K^{0}-E_{1}(\mathbf{q} ; \mathbf{K})-E_{2}(\mathbf{q} ; \mathbf{K})\right] E_{1}(\mathbf{q} ; \mathbf{K})}{-P^{0}+K^{0}+E_{2}(\mathbf{q} ; \mathbf{K})+E_{2}(\mathbf{p} ; \mathbf{P})}\right) \\
= & i \frac{\left[P^{0}-E_{2}(\mathbf{p} ; \mathbf{P})-E_{1}(\mathbf{q} ; \mathbf{K})-E_{2}(\mathbf{q} ; \mathbf{K})\right]\left[E_{1}(\mathbf{q} ; \mathbf{K})+E_{2}(\mathbf{q} ; \mathbf{K})\right]-K^{0} E_{2}(\mathbf{q} ; \mathbf{K})}{\left[P^{0}-K^{0}-E_{2}(\mathbf{q} ; \mathbf{K})-E_{2}(\mathbf{p} ; \mathbf{P})\right]\left[P^{0}-E_{1}(\mathbf{q} ; \mathbf{K})-E_{2}(\mathbf{p} ; \mathbf{P})\right]}
\end{aligned}
$$

$$
\begin{aligned}
\Xi_{2}^{+} & (\mathbf{q}, \mathbf{p} ; K, P)= \\
= & \frac{i}{K^{0}+E_{1}(\mathbf{q} ; \mathbf{K})-E_{2}(\mathbf{q} ; \mathbf{K})} \\
& \times\left(\frac{\left[K^{0}-E_{1}(\mathbf{q} ; \mathbf{K})-E_{2}(\mathbf{q} ; \mathbf{K})\right] E_{2}(\mathbf{q} ; \mathbf{K})}{-P^{0}-E_{1}(\mathbf{q} ; \mathbf{K})-E_{2}(\mathbf{p} ; \mathbf{P})}+\frac{\left[K^{0}+E_{1}(\mathbf{q} ; \mathbf{K})+E_{2}(\mathbf{q} ; \mathbf{K})\right] E_{1}(\mathbf{q} ; \mathbf{K})}{-P^{0}+K^{0}-E_{2}(\mathbf{q} ; \mathbf{K})-E_{2}(\mathbf{p} ; \mathbf{P})}\right) \\
= & -i \frac{\left[P^{0}+E_{2}(\mathbf{p} ; \mathbf{P})+E_{1}(\mathbf{q} ; \mathbf{K})+E_{2}(\mathbf{q} ; \mathbf{K})\right]\left[E_{1}(\mathbf{q} ; \mathbf{K})+E_{2}(\mathbf{q} ; \mathbf{K})\right]-K^{0} E_{2}(\mathbf{q} ; \mathbf{K})}{\left[P^{0}-K^{0}+E_{2}(\mathbf{q} ; \mathbf{K})+E_{2}(\mathbf{p} ; \mathbf{P})\right]\left[P^{0}+E_{1}(\mathbf{q} ; \mathbf{K})+E_{2}(\mathbf{p} ; \mathbf{P})\right]} .
\end{aligned}
$$

Non-relativistic approximation. In the following, we consider the CM frame, $\mathbf{K}=0$, as in section 5 . We shall evaluate $\Xi_{1}(\mathbf{q}, \mathbf{p} ; K, P)$ and $\Xi_{2}(\mathbf{q}, \mathbf{p} ; K, P)$ at next-to-leading order in the momenta $\mathbf{q}, \mathbf{p}$, applying the non-relativistic approximations of eqs. (C.18)-(C.20), and setting, according to eq. (C.21),

$$
\begin{aligned}
& K^{0}=m+\frac{\mathbf{K}^{2}}{2 m}+\mathcal{E}_{\mathbf{k}}, \\
& P^{0}=m+\frac{\mathbf{P}^{2}}{2 m}+\mathcal{E}_{n},
\end{aligned}
$$

where $\mathcal{E}_{n}$ and $\mathcal{E}_{\mathbf{k}}$ are given by eqs. (F.7) and (F.13). The next-to-leading order corrections in $\mathbf{q}, \mathbf{p}$ become important when the leading term in a $v_{\text {rel }}^{2}$ expansion cancels, as is the case for the interaction of two degenerate scalar particles via a scalar mediator (cf. section 5.1). Note though that we drop subleading terms in the couplings; such corrections do not change the structure of the wavefunction convolution integrals (cf. eqs. (5.7), (5.8)) that enter into the transition amplitudes of section 5 , and thus do not avert the cancellation of the leading 
term in the $v_{\text {rel }}^{2}$ expansion. The same holds for $\mathbf{P}^{2}$ corrections. In addition, as seen from eq. (3.41), $\mathbf{P}^{2}$ corrections are of order $v_{\text {rel }}^{4}, \alpha^{4}$ and $\alpha^{2} v_{\text {rel }}^{2}$, while $\mathbf{p}^{2}$ corrections are only of order $v_{\text {rel }}^{2}$. Similarly, $\mathbf{P} \cdot \mathbf{p}$ corrections are suppressed with respect to $\mathbf{p}^{2}$. As mentioned, we are interested, in particular, in evaluating $\Xi_{1}(\mathbf{q}, \mathbf{p} ; K, P)$ at $\mathbf{q}-\mathbf{p}-\eta_{2} \mathbf{P}_{\varphi}=0$ and $\Xi_{2}(\mathbf{q}, \mathbf{p} ; K, P)$ at $\mathbf{q}-\mathbf{p}+\eta_{1} \mathbf{P}_{\varphi}=0$. Then, according to the above, we shall keep only the $\mathrm{p}^{2}$ corrections.

At $\mathbf{q}-\mathbf{p}-\eta_{2} \mathbf{P}_{\varphi}=0$, we find

$$
\Xi_{1}(\mathbf{q}, \mathbf{p} ; K, P) \simeq 2 m_{2} \mathcal{S}_{0}(\mathbf{q} ; K) \mathcal{S}_{0}(\mathbf{p} ; P)\left[1+\frac{\mathbf{p}^{2}}{2 \mu^{2}}\left(1-\frac{2 \mu}{m}\right)\right]
$$

and at $\mathbf{q}-\mathbf{p}+\eta_{1} \mathbf{P}_{\varphi}=0$, we find

$$
\Xi_{2}(\mathbf{q}, \mathbf{p} ; K, P) \simeq 2 m_{1} \mathcal{S}_{0}(\mathbf{q} ; K) \mathcal{S}_{0}(\mathbf{p} ; P)\left[1+\frac{\mathbf{p}^{2}}{2 \mu^{2}}\left(1-\frac{2 \mu}{m}\right)\right]
$$

\section{F Schrödinger wavefunctions and convolution integrals}

In the following, we shall consider the attractive Coulomb potential

$$
V(\mathbf{r})=-\frac{\alpha}{r}
$$

\section{F.1 Solutions of the Schrödinger equation}

The discrete spectrum of solutions to the Schrödinger equation (2.84),

$$
\left[-\frac{\nabla^{2}}{2 \mu}+V(\mathbf{r})\right] \psi_{n}(\mathbf{r})=\mathcal{E}_{n} \psi_{n}(\mathbf{r})
$$

for the Coulomb potential of eq. (F.1), are (see e.g. [76])

$$
\begin{aligned}
\psi_{n \ell m}(\mathbf{r}) & =R_{n \ell}(r) Y_{\ell m}(\Omega), \\
R_{n \ell}(r) & =(2 \kappa / n)^{3 / 2}\left[\frac{(n-\ell-1) !}{2 n(n+\ell) !}\right]^{1 / 2} e^{-\kappa r / n}(2 \kappa r / n)^{\ell} L_{n-\ell-1}^{(2 \ell+1)}(2 \kappa r / n),
\end{aligned}
$$

where $Y_{\ell m}(\Omega)$ are the spherical harmonics and $L_{n-\ell-1}$ are the generalized Laguerre polynomials of degree $n-\ell-1 .{ }^{18}$ In the above,

$$
\kappa \equiv \mu \alpha
$$

is the Bohr momentum, and the energy eigenvalues are

$$
\mathcal{E}_{n}=-\frac{\mu \alpha^{2}}{2 n^{2}} .
$$

\footnotetext{
${ }^{18}$ We assume the following normalisation for the Laguerre polynomials

$$
\int_{0}^{\infty} x^{a} e^{-x} L_{n}^{(a)}(x) L_{m}^{(a)}(x) d x=\frac{\Gamma(n+a+1)}{n !} \delta_{n, m}
$$
}


In the following, we shall consider transitions to the ground state $\{100\}$. For the case of a scalar mediator, we shall also consider radiative capture to the first excited state with non-zero angular momentum $\{210\}$. The corresponding wavefuctions are

$$
\begin{aligned}
& \psi_{100}(\mathbf{r})=\frac{\kappa^{3 / 2}}{\sqrt{\pi}} e^{-\kappa r}, \\
& \psi_{210}(\mathbf{r})=\frac{\kappa^{3 / 2}}{4 \sqrt{2 \pi}} \kappa r e^{-\kappa r / 2} \cos \theta_{\mathbf{r}},
\end{aligned}
$$

where $\theta_{\mathbf{r}}$ is the polar angle of the position vector $\mathbf{r}$.

The continuous spectrum of solutions to the Schrödinger equation (2.85)

$$
\left[-\frac{\nabla^{2}}{2 \mu}+V(\mathbf{r})\right] \phi_{\mathbf{k}}(\mathbf{r})=\mathcal{E}_{\mathbf{k}} \phi_{\mathbf{k}}(\mathbf{r})
$$

is characterized by the quantum number $\mathbf{k}$, which is the expectation value of the momentum of the reduced system, with $\mathbf{k}=\mu \mathbf{v}_{\text {rel }}$ and $\mathbf{v}_{\text {rel }}$ being the expectation value of the relative velocity. The solutions are (see e.g. [76])

$$
\phi_{\mathbf{k}}(\mathbf{r})=e^{\pi \zeta / 2} \Gamma(1-i \zeta) F[i \zeta, 1, i(k r-\mathbf{k} \cdot \mathbf{r})] e^{i \mathbf{k} \cdot \mathbf{r}},
$$

where $k=|\mathbf{k}|$, and

$$
\zeta \equiv \kappa / k
$$

The energy eigenvalues are

$$
\mathcal{E}_{\mathbf{k}}=\frac{\mathbf{k}^{2}}{2 \mu}=\frac{1}{2} \mu v_{\text {rel }}^{2}
$$

Thus, $\zeta=\alpha / v_{\text {rel }}$.

\section{F.2 Convolution of the wavefunctions}

We now want to calculate the integrals (5.7)-(5.9), appearing in the amplitudes of section 5 ,

$$
\begin{aligned}
\mathcal{I}_{\mathbf{k}, n}(\mathbf{b}) & \equiv \int \frac{d^{3} p}{(2 \pi)^{3}} \tilde{\psi}_{n}^{\star}(\mathbf{p}) \tilde{\phi}_{\mathbf{k}}(\mathbf{p}+\mathbf{b})=\int d^{3} r \psi_{n}^{\star}(\mathbf{r}) \phi_{\mathbf{k}}(\mathbf{r}) e^{-i \mathbf{b} \cdot \mathbf{r}}, \\
\mathcal{J}_{\mathbf{k}, n}(\mathbf{b}) & \equiv \int \frac{d^{3} p}{(2 \pi)^{3}} \mathbf{p} \tilde{\psi}_{n}^{\star}(\mathbf{p}) \tilde{\phi}_{\mathbf{k}}(\mathbf{p}+\mathbf{b})=i \int d^{3} r\left[\nabla \psi_{n}^{\star}(\mathbf{r})\right] \phi_{\mathbf{k}}(\mathbf{r}) e^{-i \mathbf{b} \cdot \mathbf{r}}, \\
\mathcal{K}_{\mathbf{k}, n}(\mathbf{b}) & \equiv \int \frac{d^{3} p}{(2 \pi)^{3}} \mathbf{p}^{2} \tilde{\psi}_{n}^{\star}(\mathbf{p}) \tilde{\phi}_{\mathbf{k}}(\mathbf{p}+\mathbf{b})=-\int d^{3} r\left[\nabla^{2} \psi_{n}^{\star}(\mathbf{r})\right] \phi_{\mathbf{k}}(\mathbf{r}) e^{-i \mathbf{b} \cdot \mathbf{r}},
\end{aligned}
$$

and

$$
\begin{aligned}
\mathcal{I}_{n^{\prime}, n}(\mathbf{b}) & \equiv \int \frac{d^{3} p}{(2 \pi)^{3}} \tilde{\psi}_{n}^{\star}(\mathbf{p}) \tilde{\psi}_{n^{\prime}}(\mathbf{p}+\mathbf{b})=\int d^{3} r \psi_{n}^{\star}(\mathbf{r}) \psi_{n^{\prime}}(\mathbf{r}) e^{-i \mathbf{b} \cdot \mathbf{r}} \\
\mathcal{J}_{n^{\prime}, n}(\mathbf{b}) & \equiv \int \frac{d^{3} p}{(2 \pi)^{3}} \mathbf{p} \tilde{\psi}_{n}^{\star}(\mathbf{p}) \tilde{\psi}_{n^{\prime}}(\mathbf{p}+\mathbf{b})=i \int d^{3} r\left[\nabla \psi_{n}^{\star}(\mathbf{r})\right] \psi_{n^{\prime}}(\mathbf{r}) e^{-i \mathbf{b} \cdot \mathbf{r}} \\
\mathcal{K}_{n^{\prime}, n}(\mathbf{b}) & \equiv \int \frac{d^{3} p}{(2 \pi)^{3}} \mathbf{p}^{2} \tilde{\psi}_{n}^{\star}(\mathbf{p}) \tilde{\psi}_{n^{\prime}}(\mathbf{p}+\mathbf{b})=-\int d^{3} r\left[\nabla^{2} \psi_{n}^{\star}(\mathbf{r})\right] \psi_{n^{\prime}}(\mathbf{r}) e^{-i \mathbf{b} \cdot \mathbf{r}}
\end{aligned}
$$

where we transformed into the coordinate space using eqs. (2.82) and (2.83). 
For the $\{100\}$ state, the integrals (F.14)-(F.16) become

$$
\begin{aligned}
& \mathcal{I}_{\mathbf{k},\{100\}}(\mathbf{b})=\frac{\kappa^{3 / 2} e^{\pi \zeta / 2} \Gamma(1-i \zeta)}{\sqrt{\pi}} \int d^{3} r e^{i(\mathbf{k}-\mathbf{b}) \cdot \mathbf{r}-\kappa r} F[i \zeta, 1, i(k r-\mathbf{k} \cdot \mathbf{r})], \\
& \mathcal{J}_{\mathbf{k},\{100\}}(\mathbf{b})=-\frac{i \kappa^{5 / 2} e^{\pi \zeta / 2} \Gamma(1-i \zeta)}{\sqrt{\pi}} \int d^{3} r \hat{\mathbf{r}} e^{i(\mathbf{k}-\mathbf{b}) \cdot \mathbf{r}-\kappa r} F[i \zeta, 1, i(k r-\mathbf{k} \cdot \mathbf{r})], \\
& \mathcal{K}_{\mathbf{k},\{100\}}(\mathbf{b})=-\kappa^{2} \mathcal{I}_{\mathbf{k}, n=1}(\mathbf{b})+\overline{\mathcal{K}}_{\mathbf{k}}(\mathbf{b}),
\end{aligned}
$$

where $\hat{\mathbf{r}}=\mathbf{r} / r$ and

$$
\overline{\mathcal{K}}_{\mathbf{k}}(\mathbf{b}) \equiv \frac{2 \kappa^{5 / 2} e^{\pi \zeta / 2} \Gamma(1-i \zeta)}{\sqrt{\pi}} \int \frac{d^{3} r}{r} e^{i(\mathbf{k}-\mathbf{b}) \cdot \mathbf{r}-\kappa r} F[i \zeta, 1, i(k r-\mathbf{k} \cdot \mathbf{r})] .
$$

For the $\{210\}$ state, we will need only the integral (F.14),

$$
\mathcal{I}_{\mathbf{k},\{210\}}(\mathbf{b})=\frac{\kappa^{5 / 2} e^{\pi \zeta / 2} \Gamma(1-i \zeta)}{4 \sqrt{2 \pi}} \int d^{3} r r \cos \theta_{\mathbf{r}} e^{i(\mathbf{k}-\mathbf{b}) \cdot \mathbf{r}-\kappa r / 2} F[i \zeta, 1, i(k r-\mathbf{k} \cdot \mathbf{r})] .
$$

To evaluate these expressions, we make use of the identity [64]

$$
\int d^{3} r \frac{e^{i(\mathbf{k}-\mathbf{b}) \cdot \mathbf{r}-\kappa r}}{4 \pi r} F[i \zeta, 1, i(k r-\mathbf{k} \cdot \mathbf{r})]=\frac{\left[\mathbf{b}^{2}+(\kappa-i k)^{2}\right]^{-i \zeta}}{\left[(\mathbf{k}-\mathbf{b})^{2}+\kappa^{2}\right]^{1-i \zeta}} \equiv f_{\mathbf{k}, \mathbf{b}}(\kappa) .
$$

Differentiating eq. (F.25) with respect to $\kappa$ [29], with respect to $b^{j}$ [64], and with respect to $b$, we obtain the following expressions

$$
\begin{aligned}
\mathcal{I}_{\mathbf{k},\{100\}}(\mathbf{b}) & =-4 \sqrt{\pi} e^{\pi \zeta / 2} \Gamma(1-i \zeta) \kappa^{3 / 2} \frac{\partial f_{\mathbf{k}, \mathbf{b}}(\kappa)}{\partial \kappa}, \\
\mathcal{J}_{\mathbf{k},\{100\}}^{j}(\mathbf{b}) & =4 \sqrt{\pi} e^{\pi \zeta / 2} \Gamma(1-i \zeta) \kappa^{5 / 2} \frac{\partial f_{\mathbf{k}, \mathbf{b}}(\kappa)}{\partial b^{j}}, \\
\overline{\mathcal{K}}_{\mathbf{k}}(\mathbf{b}) & =8 \sqrt{\pi} e^{\pi \zeta / 2} \Gamma(1-i \zeta) \kappa^{5 / 2} f_{\mathbf{k}, \mathbf{b}}(\kappa) . \\
\mathcal{I}_{\mathbf{k},\{210\}}(\mathbf{b}) & =-i \sqrt{\frac{\pi}{2}} e^{\pi \zeta / 2} \Gamma(1-i \zeta) \kappa^{5 / 2}\left[\frac{\partial^{2} f_{\mathbf{k}, \mathbf{b}}\left(\kappa^{\prime}\right)}{\partial b \partial \kappa^{\prime}}\right]_{\kappa^{\prime}=\kappa / 2} .
\end{aligned}
$$

For the cases of interest, $\mathbf{b}=\eta_{2} \mathbf{P}_{\varphi}$ or $-\eta_{1} \mathbf{P}_{\varphi}$; evidently $b=|\mathbf{b}|<\left|\mathbf{P}_{\varphi}\right|$. Moreover, $\left|\mathbf{P}_{\varphi}\right|$ is determined by energy-momentum conservation (cf. eq. (3.41)). In the CM frame $(\mathbf{K}=0)$, and in the non-relativistic regime, $\left|\mathbf{P}_{\varphi}\right| \simeq \mu\left(\alpha^{2}+v_{\text {rel }}^{2}\right) / 2$ for capture in the ground state, and $\left|\mathbf{P}_{\varphi}\right| \simeq \mu\left(\alpha^{2} / 4+v_{\text {rel }}^{2}\right) / 2$ for capture in the $\{210\}$ state. As long as $\alpha, v_{\text {rel }}<1$, then $b \ll \kappa$ if $\alpha>v_{\text {rel }}$, or $b \ll k$, if $\alpha<v_{\text {rel }}$. In evaluating eqs. (F.26)-(F.29), we may thus expand in $b$ and keep only the leading orders. In particular, to estimate $\partial f_{\mathbf{k}, \mathbf{b}}(\kappa) / \partial \kappa$, $\partial f_{\mathbf{k}, \mathbf{b}}(\kappa) / \partial b^{j}$ and $\partial^{2} f_{\mathbf{k}, \mathbf{b}}\left(\kappa^{\prime}\right) / \partial b \partial \kappa^{\prime}$, we first differentiate $f$, then use $\kappa=\zeta k$ (cf. eq. (F.12)) and expand up to order $b^{2}$ around $b=0$. (Note, though, that in most applications, we will not need all of the terms included in the expansions below.) Setting

$$
\cos \tilde{\theta} \equiv \frac{\mathbf{k} \cdot \mathbf{b}}{k b},
$$


we find

$$
\begin{aligned}
f_{\mathbf{k}, \mathbf{b}}(\kappa) \simeq & \frac{1}{k^{2}\left(1+\zeta^{2}\right)^{2}}\left(\frac{i \zeta+1}{i \zeta-1}\right)^{-i \zeta} \\
& \times\left[1+\frac{2 b \cos \tilde{\theta}}{k(1+i \zeta)}+\frac{b^{2}\left[-(1-i \zeta)+2(2-i \zeta) \cos ^{2} \tilde{\theta}\right]}{k^{2}\left(1+\zeta^{2}\right)(1+i \zeta)}\right], \\
\frac{\partial f_{\mathbf{k}, \mathbf{b}}(\kappa)}{\partial \kappa} \simeq & -\frac{4 \zeta b}{k^{4}\left(1+\zeta^{2}\right)^{2}(1+i \zeta)}\left(\frac{i \zeta+1}{i \zeta-1}\right)^{-i \zeta} \\
& \times\left[\cos \tilde{\theta}+\frac{b\left[-(1-i \zeta)+2(2-i \zeta) \cos ^{2} \tilde{\theta}\right]}{k\left(1+\zeta^{2}\right)}\right], \\
\frac{\partial f_{\mathbf{k}, \mathbf{b}}(\kappa)}{\partial b^{j}} \simeq & \frac{2}{k^{4}\left(1+\zeta^{2}\right)(1+i \zeta)}\left(\frac{i \zeta+1}{i \zeta-1}\right)^{-i \zeta}\left\{-\frac{b^{j}}{1+i \zeta}\left[1+\frac{4 b \cos \tilde{\theta}}{k\left(1+\zeta^{2}\right)}\right]\right. \\
& \left.+k^{j}\left[1+\frac{2(2-i \zeta) b \cos \tilde{\theta}}{k\left(1+\zeta^{2}\right)}-\frac{\left.2 b^{2}\left[1-i \zeta-\left(6-\zeta^{2}-i 5 \zeta\right) \cos ^{2} \theta\right]\right]}{k^{2}\left(1+\zeta^{2}\right)^{2}}\right]\right\},
\end{aligned}
$$

and

$$
\begin{aligned}
{\left[\frac{\partial^{2} f_{\mathbf{k}, \mathbf{b}}\left(\kappa^{\prime}\right)}{\partial b \partial \kappa^{\prime}}\right]_{\kappa^{\prime}=\kappa / 2} \simeq } & \frac{2^{8} b \zeta(i \zeta-2)}{k^{5}\left(4+\zeta^{2}\right)^{4}}\left(\frac{i \zeta+2}{i \zeta-2}\right)^{-i \zeta} \\
& \times\left[i \zeta-2-4(i \zeta-1) \cos ^{2} \tilde{\theta}\right. \\
& \left.-\frac{\left.8 b(i \zeta-1)\left[3(i \zeta-2)-4(i \zeta-3) \cos ^{2} \tilde{\theta}\right] \cos \tilde{\theta}\right]}{k\left(4+\zeta^{2}\right)}\right] .
\end{aligned}
$$

For convenience, we define

$$
\mathcal{R}(\zeta) \equiv \frac{8 \sqrt{\pi} \zeta^{5 / 2} e^{\pi \zeta / 2}}{1+\zeta^{2}} \frac{\Gamma(1-i \zeta)}{1+i \zeta}\left(\frac{i \zeta+1}{i \zeta-1}\right)^{-i \zeta}
$$

In evaluating the cross-sections of interest, we shall need

$$
|\mathcal{R}(\zeta)|^{2}=\frac{2^{7} \pi^{2} \zeta^{6}}{\left(1+\zeta^{2}\right)^{3}} \frac{e^{-4 \zeta \operatorname{arccot} \zeta}}{1-e^{-2 \pi \zeta}}
$$

where we used the identities

$$
\begin{aligned}
|\Gamma(1-i \zeta)|^{2} & =2 \pi \zeta \frac{e^{-\pi \zeta}}{1-e^{-2 \pi \zeta}} \\
\left|\left(\frac{i \zeta+1}{i \zeta-1}\right)^{-i \zeta}\right|^{2} & =e^{-4 \zeta \operatorname{arccot} \zeta} .
\end{aligned}
$$


Collecting the above, we obtain

$$
\begin{aligned}
\mathcal{I}_{\mathbf{k},\{100\}}(\mathbf{b}) \simeq & \frac{2 \mathcal{R}(\zeta)}{1+\zeta^{2}} \frac{b}{k^{5 / 2}}\left(\cos \tilde{\theta}+\frac{b\left[-1+i \zeta+2(2-i \zeta) \cos ^{2} \tilde{\theta}\right]}{k\left(1+\zeta^{2}\right)}\right) \\
\mathcal{J}_{\mathbf{k},\{100\}}(\mathbf{b}) \simeq & \frac{\mathcal{R}(\zeta)}{k^{3 / 2}}\left\{-\frac{\mathbf{b}}{1+i \zeta}\left[1+\frac{4 b \cos \tilde{\theta}}{k\left(1+\zeta^{2}\right)}\right]\right. \\
& \left.+\mathbf{k}\left[1+\frac{2(2-i \zeta) b \cos \tilde{\theta}}{k\left(1+\zeta^{2}\right)}+\frac{2 b^{2}\left[-1+i \zeta+\left(6-\zeta^{2}-i 5 \zeta\right) \cos ^{2} \tilde{\theta}\right]}{k^{2}\left(1+\zeta^{2}\right)^{2}}\right]\right\} \\
\overline{\mathcal{K}}_{\mathbf{k}}(\mathbf{b}) \simeq & \mathcal{R}(\zeta) k^{1 / 2}\left[1+i \zeta+\frac{2 b \cos \tilde{\theta}}{k}+\frac{b^{2}\left[-1+i \zeta+2(2-i \zeta) \cos ^{2} \tilde{\theta}\right]}{k^{2}\left(1+\zeta^{2}\right)}\right]
\end{aligned}
$$

and

$$
\begin{aligned}
\mathcal{I}_{\mathbf{k},\{210\}}(\mathbf{b}) \simeq & -\frac{i 2^{7} \sqrt{2 \pi} b}{k^{5 / 2}} \frac{\zeta^{7 / 2}(i \zeta-2) e^{\pi \zeta / 2} \Gamma(1-i \zeta)}{\left(4+\zeta^{2}\right)^{4}}\left(\frac{i \zeta+2}{i \zeta-2}\right)^{-i \zeta} \\
& \times\left[i \zeta-2-4(i \zeta-1) \cos ^{2} \tilde{\theta}-\frac{8 b(i \zeta-1)\left[3(i \zeta-2)-4(i \zeta-3) \cos ^{2} \tilde{\theta}\right] \cos \tilde{\theta}}{k\left(4+\zeta^{2}\right)}\right] .
\end{aligned}
$$

Note that in the above, the angle $\tilde{\theta}$ is related to the angle $\theta$ between $\mathbf{k}$ and $\mathbf{P}_{\varphi}$, defined by

$$
\cos \theta=\frac{\mathbf{k} \cdot \mathbf{P}_{\varphi}}{k\left|\mathbf{P}_{\varphi}\right|}
$$

as follows

$$
\tilde{\theta}= \begin{cases}\theta, & \text { for } \mathbf{b}=\eta_{2} \mathbf{P}_{\varphi}, \\ \pi+\theta, & \text { for } \mathbf{b}=-\eta_{1} \mathbf{P}_{\varphi}\end{cases}
$$

For the computation of the $\{210\}$ state de-excitation rate (section 5.1.3), we will need the integral $\mathcal{I}_{\{210\},\{100\}}(\mathbf{b})$. Starting from eq. (F.17), and using the wavefunctions (F.8) and (F.9), it is easy to show that

$$
\mathcal{I}_{\{210\},\{100\}}(\mathbf{b})=-\frac{i 2^{6} \sqrt{2}}{3^{4}} \frac{(2 b) /(3 \kappa)}{[1+(2 b) /(3 \kappa)]^{3}} \simeq-\frac{i 2^{7} \sqrt{2} b}{3^{5} \kappa},
$$

where we took into account that in the $\{210\} \rightarrow\{100\}$ radiative transition, the mediator is emitted with momentum $\left|\mathbf{P}_{\varphi}\right|=(3 / 8) \mu \alpha^{2}$; then, for $b=\eta_{1,2}\left|\mathbf{P}_{\varphi}\right|, 2 b /(3 \kappa)=\eta_{1,2} \alpha / 4 \ll 1$.

Open Access. This article is distributed under the terms of the Creative Commons Attribution License (CC-BY 4.0), which permits any use, distribution and reproduction in any medium, provided the original author(s) and source are credited. 


\section{References}

[1] D.N. Spergel and P.J. Steinhardt, Observational evidence for selfinteracting cold dark matter, Phys. Rev. Lett. 84 (2000) 3760 [astro-ph/9909386] [INSPIRE].

[2] A. Kusenko and P.J. Steinhardt, Q ball candidates for selfinteracting dark matter, Phys. Rev. Lett. 87 (2001) 141301 [astro-ph/0106008] [INSPIRE].

[3] J.L. Feng, H. Tu and H.-B. Yu, Thermal relics in hidden sectors, JCAP 10 (2008) 043 [arXiv:0808.2318] [INSPIRE].

[4] A. Loeb and N. Weiner, Cores in dwarf galaxies from dark matter with a Yukawa potential, Phys. Rev. Lett. 106 (2011) 171302 [arXiv:1011.6374] [INSPIRE].

[5] D.H. Weinberg, J.S. Bullock, F. Governato, R.K. de Naray and A.H.G. Peter, Cold dark matter: controversies on small scales, arXiv:1306.0913 [INSPIRE].

[6] A.H.G. Peter, M. Rocha, J.S. Bullock and M. Kaplinghat, Cosmological simulations with self-interacting dark matter II: halo shapes vs. observations, Mon. Not. Roy. Astron. Soc. 430 (2013) 105 [arXiv: 1208.3026] [INSPIRE].

[7] M. Rocha et al., Cosmological simulations with self-interacting dark matter I: constant density cores and substructure, Mon. Not. Roy. Astron. Soc. 430 (2013) 81 [arXiv: 1208.3025] [INSPIRE].

[8] M. Vogelsberger, J. Zavala, C. Simpson and A. Jenkins, Dwarf galaxies in CDM and SIDM with baryons: observational probes of the nature of dark matter, Mon. Not. Roy. Astron. Soc. 444 (2014) 3684 [arXiv:1405.5216] [INSPIRE].

[9] J. Zavala, M. Vogelsberger and M.G. Walker, Constraining self-interacting dark matter with the Milky Way's dwarf spheroidals, Monthly Notices of the Royal Astronomical Society: Letters 431 (2013) L20 [arXiv:1211.6426] [INSPIRE].

[10] M. Vogelsberger, J. Zavala and A. Loeb, Subhaloes in self-interacting galactic dark matter haloes, Mon. Not. Roy. Astron. Soc. 423 (2012) 3740 [arXiv:1201.5892] [InSPIRE].

[11] H. Davoudiasl and R.N. Mohapatra, On relating the genesis of cosmic baryons and dark matter, New J. Phys. 14 (2012) 095011 [arXiv: 1203.1247] [INSPIRE].

[12] K. Petraki and R.R. Volkas, Review of asymmetric dark matter, Int. J. Mod. Phys. A 28 (2013) 1330028 [arXiv: 1305.4939] [INSPIRE].

[13] K.M. Zurek, Asymmetric dark matter: theories, signatures and constraints, Phys. Rept. 537 (2014) 91 [arXiv:1308.0338] [INSPIRE].

[14] S.M. Boucenna and S. Morisi, Theories relating baryon asymmetry and dark matter: A mini review, Front. Phys. 1 (2014) 33 [arXiv:1310.1904] [INSPIRE].

[15] R. Foot and R.R. Volkas, Was ordinary matter synthesized from mirror matter? An attempt to explain why Omega(baryon) approximately equal to 0.2 Omega(dark), Phys. Rev. D 68 (2003) 021304 [hep-ph/0304261] [INSPIRE].

[16] D.E. Kaplan, G.Z. Krnjaic, K.R. Rehermann and C.M. Wells, Atomic dark matter, JCAP 05 (2010) 021 [arXiv:0909.0753] [INSPIRE].

[17] K. Petraki, M. Trodden and R.R. Volkas, Visible and dark matter from a first-order phase transition in a baryon-symmetric universe, JCAP 02 (2012) 044 [arXiv:1111.4786] [INSPIRE]. 
[18] B. von Harling, K. Petraki and R.R. Volkas, Affleck-Dine dynamics and the dark sector of pangenesis, JCAP 05 (2012) 021 [arXiv:1201.2200] [INSPIRE].

[19] J.M. Cline, Z. Liu and W. Xue, Millicharged atomic dark matter, Phys. Rev. D 85 (2012) 101302 [arXiv: 1201.4858] [INSPIRE].

[20] D. Fargion, M. Khlopov and C.A. Stephan, Cold dark matter by heavy double charged leptons?, Class. Quant. Grav. 23 (2006) 7305 [astro-ph/0511789] [INSPIRE].

[21] R. Foot and Z.K. Silagadze, Thin disk of co-rotating dwarfs: a fingerprint of dissipative (mirror) dark matter?, Phys. Dark Univ. 2 (2013) 163 [arXiv:1306.1305] [INSPIRE].

[22] R. Foot, Tully-Fisher relation, galactic rotation curves and dissipative mirror dark matter, JCAP 12 (2014) 047 [arXiv: 1307.1755] [INSPIRE].

[23] R. Foot, A dark matter scaling relation from mirror dark matter, Phys. Dark Univ. 5-6 (2014) 236 [arXiv:1303.1727] [INSPIRE].

[24] R. Foot and S. Vagnozzi, Dissipative hidden sector dark matter, Phys. Rev. D 91 (2015) 023512 [arXiv: 1409.7174] [INSPIRE].

[25] R. Foot, Dissipative dark matter explains rotation curves, arXiv:1502.07817 [INSPIRE].

[26] J. Fan, A. Katz, L. Randall and M. Reece, Dark-disk universe, Phys. Rev. Lett. 110 (2013) 211302 [arXiv: 1303.3271] [INSPIRE].

[27] N. Arkani-Hamed, D.P. Finkbeiner, T.R. Slatyer and N. Weiner, A theory of dark matter, Phys. Rev. D 79 (2009) 015014 [arXiv:0810.0713] [INSPIRE].

[28] M. Pospelov and A. Ritz, Astrophysical signatures of secluded dark matter, Phys. Lett. B 671 (2009) 391 [arXiv:0810.1502] [INSPIRE].

[29] L. Pearce and A. Kusenko, Indirect detection of self-interacting asymmetric dark matter, Phys. Rev. D 87 (2013) 123531 [arXiv:1303.7294] [INSPIRE].

[30] L. Pearce, K. Petraki and A. Kusenko, Signals from dark atom formation in halos, Phys. Rev. D 91 (2015) 083532 [arXiv: 1502.01755] [INSPIRE].

[31] M.T. Frandsen, F. Sannino, I.M. Shoemaker and O. Svendsen, X-ray lines from dark matter: the good, the bad and the unlikely, JCAP 05 (2014) 033 [arXiv:1403.1570] [INSPIRE].

[32] K.K. Boddy, J.L. Feng, M. Kaplinghat, Y. Shadmi and T.M.P. Tait, Strongly interacting dark matter: self-interactions and keV lines, Phys. Rev. D 90 (2014) 095016 [arXiv: 1408.6532] [INSPIRE].

[33] J.M. Cline, Y. Farzan, Z. Liu, G.D. Moore and W. Xue, $3.5 \mathrm{keV}$ x-rays as the "21 cm line" of dark atoms and a link to light sterile neutrinos, Phys. Rev. D 89 (2014) 121302 [arXiv: 1404.3729] [INSPIRE].

[34] W. Detmold, M. McCullough and A. Pochinsky, Dark nuclei I: cosmology and indirect detection, Phys. Rev. D 90 (2014) 115013 [arXiv:1406.2276] [INSPIRE].

[35] J.D. March-Russell and S.M. West, WIMPonium and boost factors for indirect dark matter detection, Phys. Lett. B 676 (2009) 133 [arXiv:0812.0559] [INSPIRE].

[36] R. Foot, Implications of the DAMA and CRESST experiments for mirror matter type dark matter, Phys. Rev. D 69 (2004) 036001 [hep-ph/0308254] [INSPIRE].

[37] N. Fornengo, P. Panci and M. Regis, Long-range forces in direct dark matter searches, Phys. Rev. D 84 (2011) 115002 [arXiv:1108.4661] [INSPIRE]. 
[38] R. Foot, Hidden sector dark matter explains the DAMA, CoGeNT, CRESST-II and CDMS/Si experiments, Phys. Rev. D 88 (2013) 025032 [arXiv:1209.5602] [InSPIRE].

[39] R. Foot, Direct detection experiments explained with mirror dark matter, Phys. Lett. B 728 (2014) 45 [arXiv: 1305.4316] [INSPIRE].

[40] J. Hisano, S. Matsumoto and M.M. Nojiri, Unitarity and higher order corrections in neutralino dark matter annihilation into two photons, Phys. Rev. D 67 (2003) 075014 [hep-ph/0212022] [INSPIRE].

[41] J. Hisano, S. Matsumoto and M.M. Nojiri, Explosive dark matter annihilation, Phys. Rev. Lett. 92 (2004) 031303 [hep-ph/0307216] [INSPIRE].

[42] M. Cirelli, A. Strumia and M. Tamburini, Cosmology and astrophysics of minimal dark matter, Nucl. Phys. B 787 (2007) 152 [arXiv:0706.4071] [inSPIRE].

[43] B. von Harling and K. Petraki, Bound-state formation for thermal relic dark matter and unitarity, JCAP 12 (2014) 033 [arXiv:1407.7874] [INSPIRE].

[44] D.E. Kaplan, G.Z. Krnjaic, K.R. Rehermann and C.M. Wells, Dark atoms: asymmetry and direct detection, JCAP 10 (2011) 011 [arXiv:1105.2073] [INSPIRE].

[45] S.R. Behbahani, M. Jankowiak, T. Rube and J.G. Wacker, Nearly supersymmetric dark atoms, Adv. High Energy Phys. 2011 (2011) 709492 [arXiv:1009. 3523] [INSPIRE].

[46] F.-Y. Cyr-Racine and K. Sigurdson, Cosmology of atomic dark matter, Phys. Rev. D 87 (2013) 103515 [arXiv: 1209.5752] [INSPIRE].

[47] J.M. Cline, Z. Liu, G. Moore and W. Xue, Scattering properties of dark atoms and molecules, Phys. Rev. D 89 (2014) 043514 [arXiv:1311.6468] [InSPIRE].

[48] K. Petraki, L. Pearce and A. Kusenko, Self-interacting asymmetric dark matter coupled to a light massive dark photon, JCAP 07 (2014) 039 [arXiv:1403.1077] [INSPIRE].

[49] K.K. Boddy, J.L. Feng, M. Kaplinghat and T.M.P. Tait, Self-interacting dark matter from a non-abelian hidden sector, Phys. Rev. D 89 (2014) 115017 [arXiv:1402.3629] [INSPIRE].

[50] G. Krnjaic and K. Sigurdson, Big Bang darkleosynthesis, arXiv:1406.1171 [INSPIRE].

[51] M.B. Wise and Y. Zhang, Stable bound states of asymmetric dark matter, Phys. Rev. D 90 (2014) 055030 [arXiv:1407.4121] [INSPIRE].

[52] M.B. Wise and Y. Zhang, Yukawa bound states of a large number of fermions, JHEP 02 (2015) 023 [arXiv:1411.1772] [INSPIRE].

[53] R. Laha and E. Braaten, Direct detection of dark matter in universal bound states, Phys. Rev. D 89 (2014) 103510 [arXiv:1311.6386] [InSPIRE].

[54] F.-Y. Cyr-Racine, R. de Putter, A. Raccanelli and K. Sigurdson, Constraints on large-scale dark acoustic oscillations from cosmology, Phys. Rev. D 89 (2014) 063517 [arXiv: 1310.3278] [INSPIRE].

[55] W. Shepherd, T.M.P. Tait and G. Zaharijas, Bound states of weakly interacting dark matter, Phys. Rev. D 79 (2009) 055022 [arXiv:0901.2125] [INSPIRE].

[56] A. Kusenko, Solitons in the supersymmetric extensions of the standard model, Phys. Lett. B 405 (1997) 108 [hep-ph/9704273] [inSPIRE].

[57] A. Kusenko and M.E. Shaposhnikov, Supersymmetric Q balls as dark matter, Phys. Lett. B 418 (1998) 46 [hep-ph/9709492] [INSPIRE]. 
[58] S. Kasuya and M. Kawasaki, Q ball formation through Affleck-Dine mechanism, Phys. Rev. D 61 (2000) 041301 [hep-ph/9909509] [INSPIRE].

[59] M. Postma, Solitosynthesis of Q balls, Phys. Rev. D 65 (2002) 085035 [hep-ph/0110199] [INSPIRE].

[60] A. Kusenko, L. Loveridge and M. Shaposhnikov, Supersymmetric dark matter Q-balls and their interactions in matter, Phys. Rev. D 72 (2005) 025015 [hep-ph/0405044] [INSPIRE].

[61] S.J. Brodsky and R.F. Lebed, Production of the smallest QED atom: true muonium $\left(\mu^{+} \mu^{-}\right)$, Phys. Rev. Lett. 102 (2009) 213401 [arXiv:0904.2225] [INSPIRE].

[62] A. Sommerfeld, Über die Beugung und Bremsung der Elektronen, Ann. Phys. 403 (1931) 257.

[63] H.A. Bethe and E.E. Salpeter, Quantum mechanics of one- and two-electron atoms, Cademic Press, New York U.S.A. (1957).

[64] A.I. Akhiezer and N.P. Merenkov, The theory of lepton bound-state production, J. Phys. C 29 (1996) 2135.

[65] R. Iengo, Sommerfeld enhancement: general results from field theory diagrams, JHEP 05 (2009) 024 [arXiv:0902.0688] [INSPIRE].

[66] S. Cassel, Sommerfeld factor for arbitrary partial wave processes, J. Phys. G 37 (2010) 105009 [arXiv: 0903.5307] [INSPIRE].

[67] C. Itzykson and J. Zuber, Quantum field theory, McGraw-Hill Education (1980).

[68] Z.K. Silagadze, Wick-Cutkosky model: an introduction, hep-ph/9803307 [INSPIRE].

[69] P. Hoyer, Bound states - From QED to QCD, arXiv:1402.5005 [INSPIRE].

[70] M.E. Peskin and D.V. Schroeder, An introduction to quantum field theory, Westview Press (1995).

[71] K. Griest and M. Kamionkowski, Unitarity limits on the mass and radius of dark matter particles, Phys. Rev. Lett. 64 (1990) 615 [INSPIRE].

[72] A. Hryczuk, The Sommerfeld enhancement for scalar particles and application to sfermion co-annihilation regions, Phys. Lett. B 699 (2011) 271 [arXiv:1102.4295] [INSPIRE].

[73] A. Hryczuk, I. Cholis, R. Iengo, M. Tavakoli and P. Ullio, Indirect detection analysis: wino dark matter case study, JCAP 07 (2014) 031 [arXiv:1401.6212] [INSPIRE].

[74] M. Beneke, C. Hellmann and P. Ruiz-Femenia, Heavy neutralino relic abundance with Sommerfeld enhancements - A study of pMSSM scenarios, JHEP 03 (2015) 162 [arXiv: 1411.6930] [INSPIRE].

[75] B. Bellazzini, M. Cliche and P. Tanedo, Effective theory of self-interacting dark matter, Phys. Rev. D 88 (2013) 083506 [arXiv:1307.1129] [INSPIRE].

[76] A. Messiah, Quantum mechanics, Elsevier, North-Holland The Netherlands (1962). 